\title{
Development of Magnetic Molecularly Imprinted Polymer Nanoparticles for Rapid and Selective Removal of Endocrine Disrupting Compounds from Aqueous Environment
}

\author{
by
}

Xinlong Xia, M.Sc.

A thesis submitted to the Faculty of Graduate and Postdoctoral Affairs in partial fulfillment of the requirements for the degree of

Master of Science

in

Chemical and Environmental Toxicology

Carleton University

Ottawa, Ontario

(c) 2012, Xinlong Xia 
Library and Archives

Canada

Published Heritage

Branch

395 Wellington Street

Ottawa ON K1A ON4

Canada
Bibliothèque et

Archives Canada

Direction du

Patrimoine de l'édition

395 , rue Wellington

Ottawa ON K1A ON4

Canada
Your file Votre référence

ISBN: 978-0-494-91509-7

Our file Notre référence

ISBN: 978-0-494-91509-7
NOTICE:

The author has granted a nonexclusive license allowing Library and Archives Canada to reproduce, publish, archive, preserve, conserve, communicate to the public by telecommunication or on the Internet, loan, distrbute and sell theses worldwide, for commercial or noncommercial purposes, in microform, paper, electronic and/or any other formats.

The author retains copyright ownership and moral rights in this thesis. Neither the thesis nor substantial extracts from it may be printed or otherwise reproduced without the author's permission.
AVIS:

L'auteur a accordé une licence non exclusive permettant à la Bibliothèque et Archives Canada de reproduire, publier, archiver, sauvegarder, conserver, transmettre au public par télécommunication ou par l'Internet, prêter, distribuer et vendre des thèses partout dans le monde, à des fins commerciales ou autres, sur support microforme, papier, électronique et/ou autres formats.

L'auteur conserve la propriété du droit d'auteur et des droits moraux qui protege cette thèse. $\mathrm{Ni}$ la thèse ni des extraits substantiels de celle-ci ne doivent être imprimés ou autrement reproduits sans son autorisation.
In compliance with the Canadian Privacy Act some supporting forms may have been removed from this thesis.

While these forms may be included in the document page count, their removal does not represent any loss of content from the thesis.
Conformément à la loi canadienne sur la protection de la vie privée, quelques formulaires secondaires ont été enlevés de cette thèse.

Bien que ces formulaires aient inclus dans la pagination, il n'y aura aucun contenu manquant. 


\section{Abstract}

The removal of endocrine disrupting compounds (EDCs) from aqueous environment was proposed using magnetic molecularly imprinted polymer (M-MIP) particles. Estrogen imprinted MIP layer was coated on magnetic core by ultrasonication assisted synthesis. The resultant particles exhibit satisfactory binding property and strong magnetic response. 17 $\beta$-estradiol - bisphenol A imprinted M-MIP offers a promising alternative for a class-specific adsorption of EDCs. A comparative study implies that magnetic separation has advantages over centrifugation and membrane filtration, facilitating an efficient, cost-effective separation of M-MIP from aqueous matrices. Environmental variables (ionic strength, humic acid, $\mathrm{pH}$ and temperature) have minor effects on M-MIP adsorption performance, showing its suitability to real water streams. The M-MIP possesses good stability and regenerating potential reusable for at least seven times without significant deterioration in recognition and magnetic properties. The simultaneous removal of EDCs was verified using river water samples spiked with five representative steroid and phenolic EDCs at a trace $(10 \mu \mathrm{g} / \mathrm{L})$ concentration level. 


\section{Acknowledgements}

My deepest thanks and respect go to my supervisor Professor Edward P.C. Lai for offering me the opportunity to research at his lab. He has demonstrated me with outstanding motivation abilities and profound knowledge. His guidance, inspiration and generous support help throughout my thesis research and Master's studies at Carleton University.

I would like to express my appreciation and thanks to the committee members, who have provided me knowledge, insight, and experience helping throughout my work.

Many thanks go to my lab mates for their help, support, friendships. I truly benefit from many of the discussions.

Finally, I would like to express my dearest thanks to my wife, daughter and son. Without their love, understanding, support and encouragement, my research and degree studies couldn't have continued. This dissertation is dedicated to my parents, whose greatest love and care have been accompanying my growth in pursuit of science knowledge. 
Table of Contents

Abstract

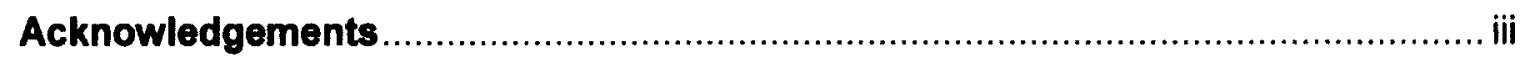

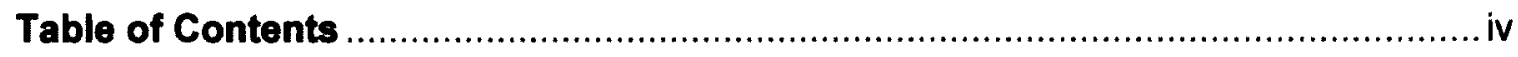

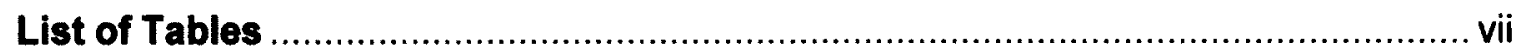

List of Figures

List of Appendices

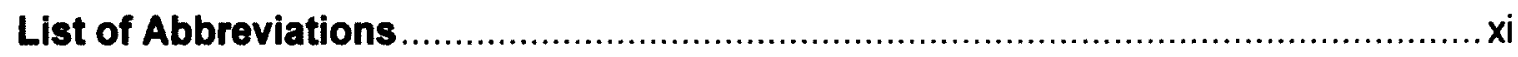

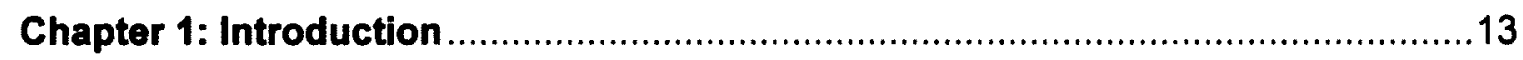

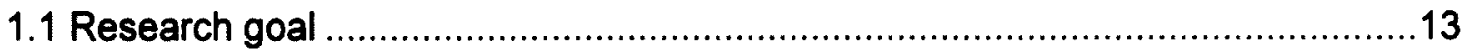

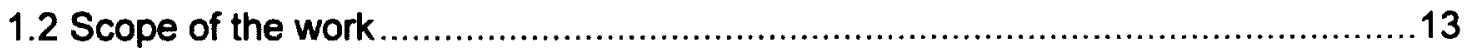

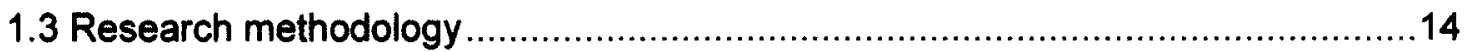

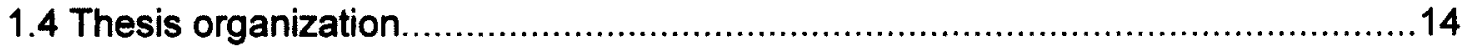

1.5 EDCs imprinted M-MIPs as selective adsorbents ......................................16

References

Chapter 2: Ultrasonication Assisted Synthesis of MIP Encapsulated Magnetic Nanoparticles for Rapid and Selective Removal of 17ß-Estradiol from

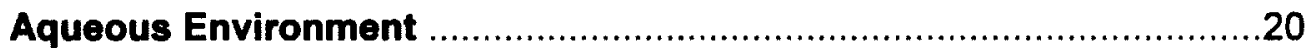

2.1 Introduction

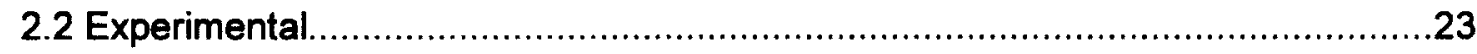

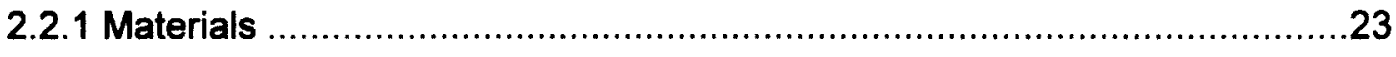

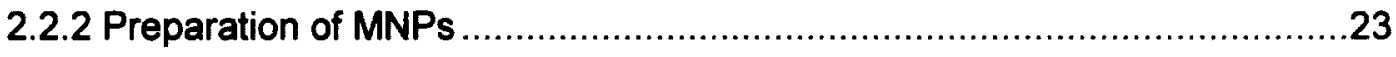

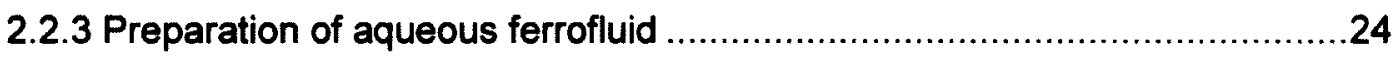

2.2.4 Preparation of pre-polymerization solution ...........................................24

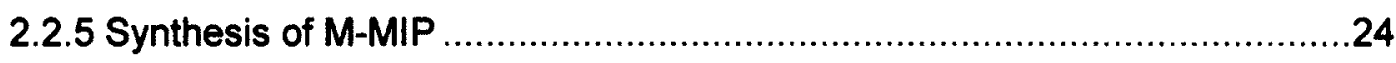




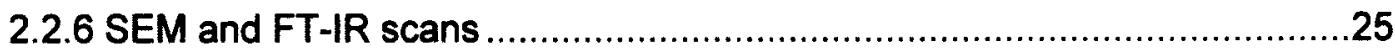

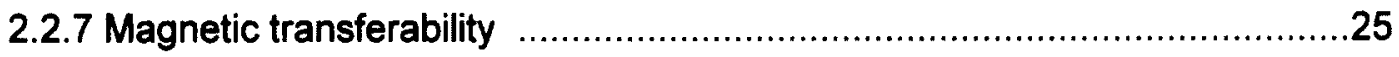

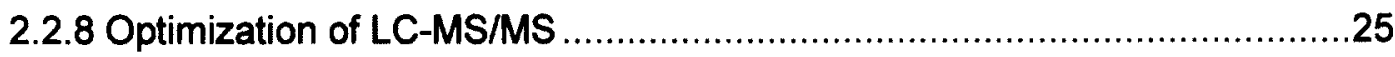

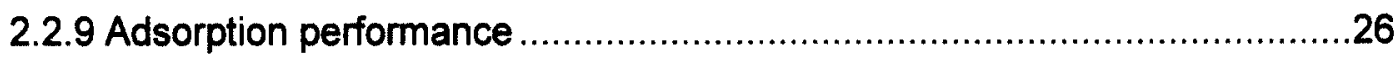

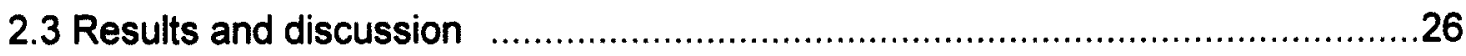

2.3.1 Synthesis of MIP encapsulated MNPs.................................................26

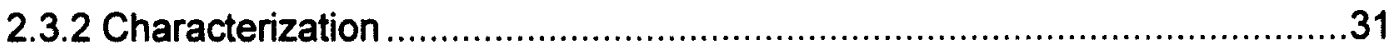

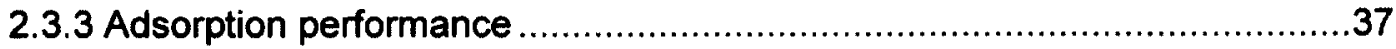

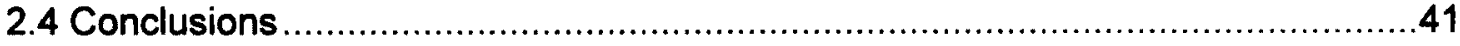

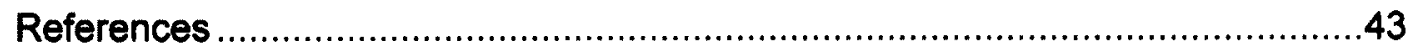

Chapter 3: Dual-Template Imprinted Magnetic Polymer Nanoparticles for ClassSpecific Removal of Endocrine Disrupting Compounds from Aqueous

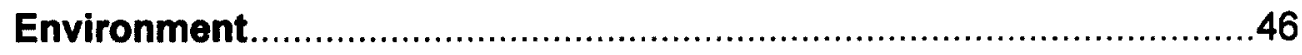

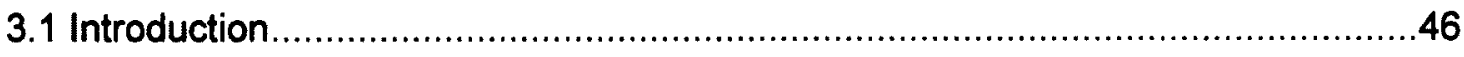

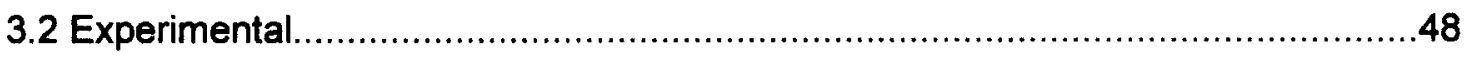

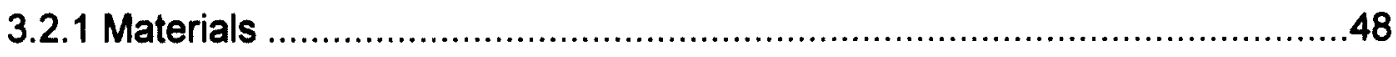

3.2.2 Synthesis of dual-template imprinted M-MIP .......................................49

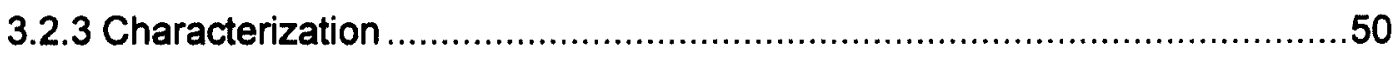

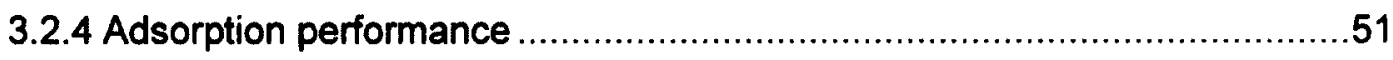

3.2.5 Adsorption tests on spiked environmental samples ................................52

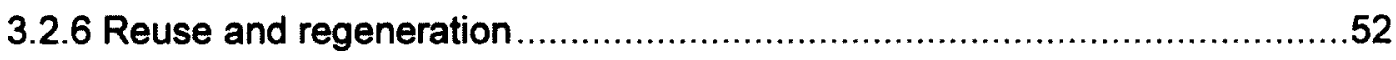

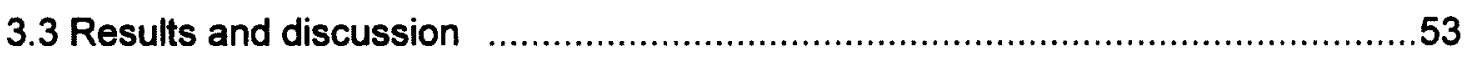

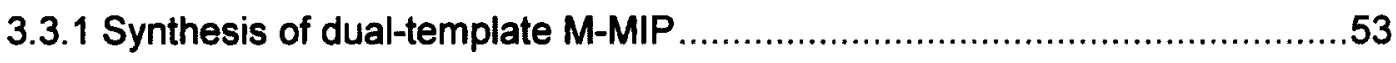

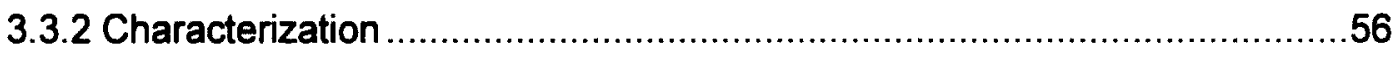

3.3.3 Magnetic separation efficiency and comparison .....................................61

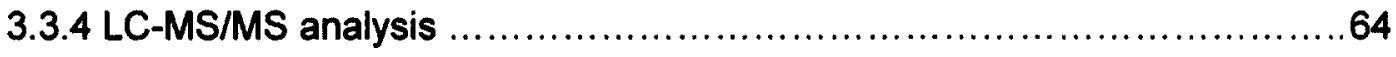


3.3.5 Effect of environmental variables on adsorption efficiency

3.3.6 Class-specific adsorption... 70

3.3.7 Application of M-MIP to environmental samples 74

3.3.8 Reuse and regeneration potential .76

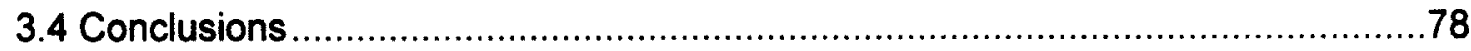

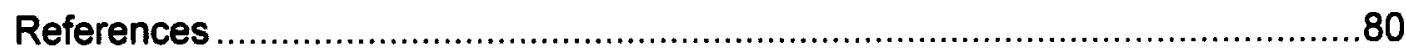

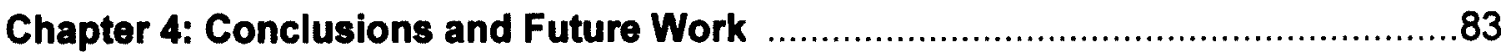

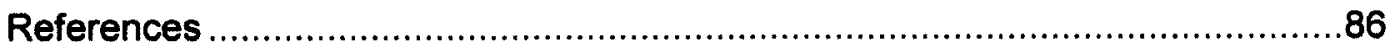




\section{List of Tables}

Table 2.1 Recovery of spiked E2 from tap water ….................................................... 41

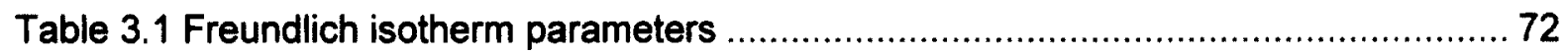




\section{List of Figures}

Fig. 1.1 Conceptual illustration of research scheme ……......................................14

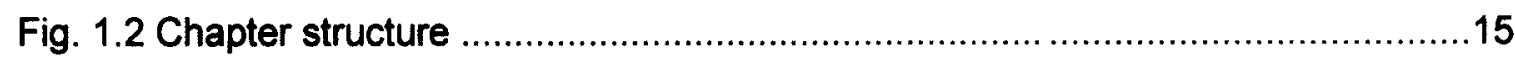

Fig. 2.1 Aqueous ferrofluid and dual-layer structure illustration ........................27

Fig. 2.2 Ultrasonication assisted synthesis of M-MIP ............................................... 30

Fig. 2.3 SEM scans of MNPs (a), MNPs-OA-SDS (b), M-NIP (c) and M-MIP (d) …......32

Fig. 2.4 FT-IR spectrum of MNPs (a) and MNPs-OA-SDS (b) ..................................3

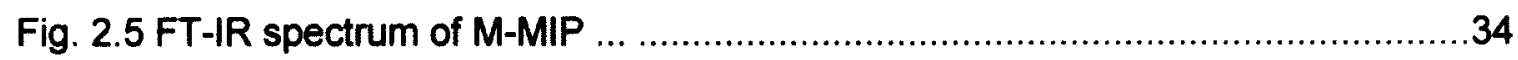

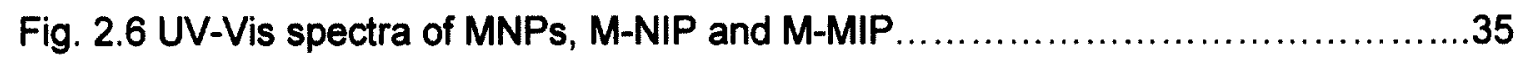

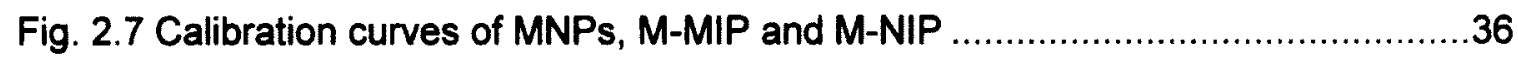

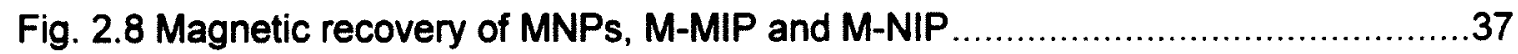

Fig. 2.9 Comparison of binding efficiency of M-MIP and M-NIP for E2 ……...............39

Fig. 2.10 Comparison of binding efficiency of M-MIP for E2 and BPA ........................40

Fig. 3.1 Structures of the studied EDCs and monomers ….......................................53

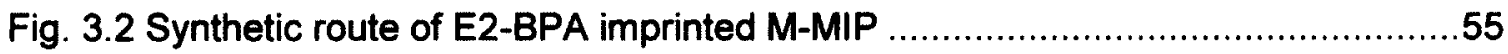

Fig. 3.3 SEM scans of $\mathrm{Fe}_{3} \mathrm{O}_{4}$-OA-SDS (a), M-NIP (b) and M-MIP (c) …....................56

Fig. 3.4 FT-IR spectrum of MAA, EGDMA and M-MIP ........................................57

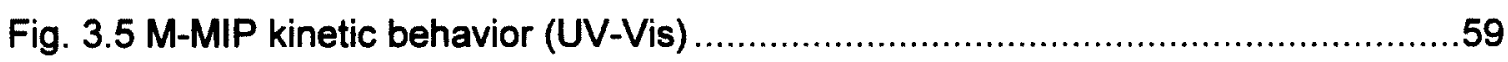

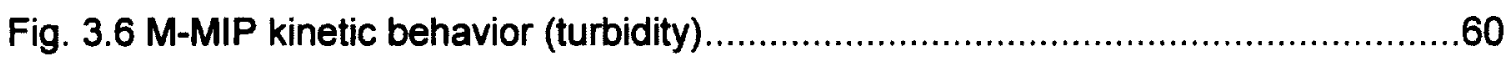

Fig. 3.7 Comparison of turbidity (a) and UV-Vis (b) measurements ..........................61

Fig. 3.8 Effect of centrifugation speed ....................................................................62

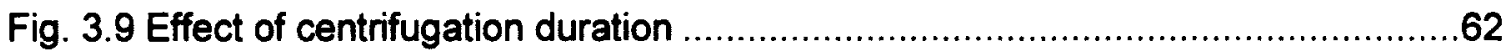

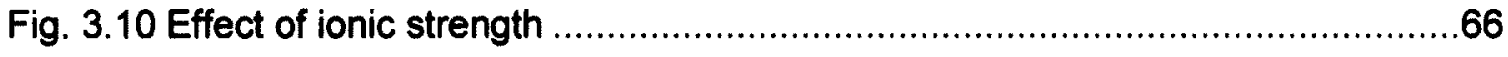

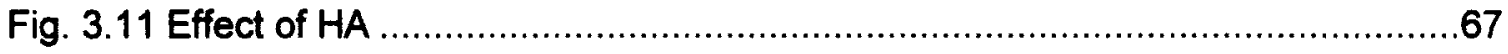

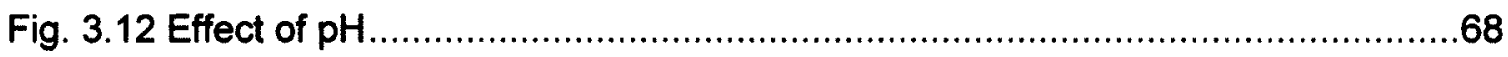




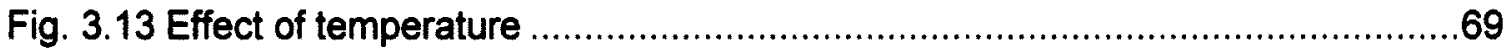

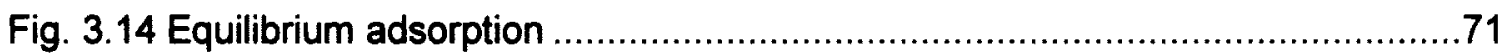

Fig. 3.15 Comparison of adsorption efficiency with co-existing EDCs ........................73

Fig. 3.16 Adsorption efficiency toward EDCs, DFC, HQ and PH...............................73

Fig. 3.17 Recovery of EDCs from spiked aqueous samples .......................................75

Fig. 3.18 Reuse and regeneration potential of M-MIP ...........................................77

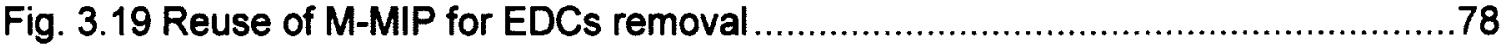




\section{List of Appendices}

A. 1 LC-MS/MS chromotogram (a) and spectrum of E3 ................................. 87

A. 2 LC-MS/MS chromotogram (a) and spectrum (b) of BPA ..............................87

A. 3 LC-MS/MS chromotogram (a) and spectrum (b) of E2 .............................88

A. 4 LC-MS/MS chromotogram (a) and spectrum (b) of E1 .............................88

A. 5 LC-MS/MS chromotogram (a) and spectrum (b) of DES ............................89

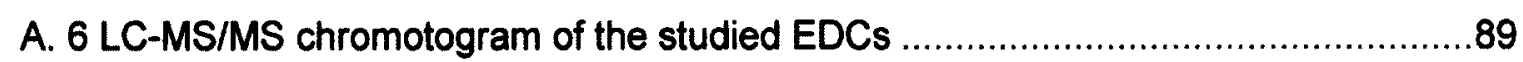




\section{List of Abbreviations}

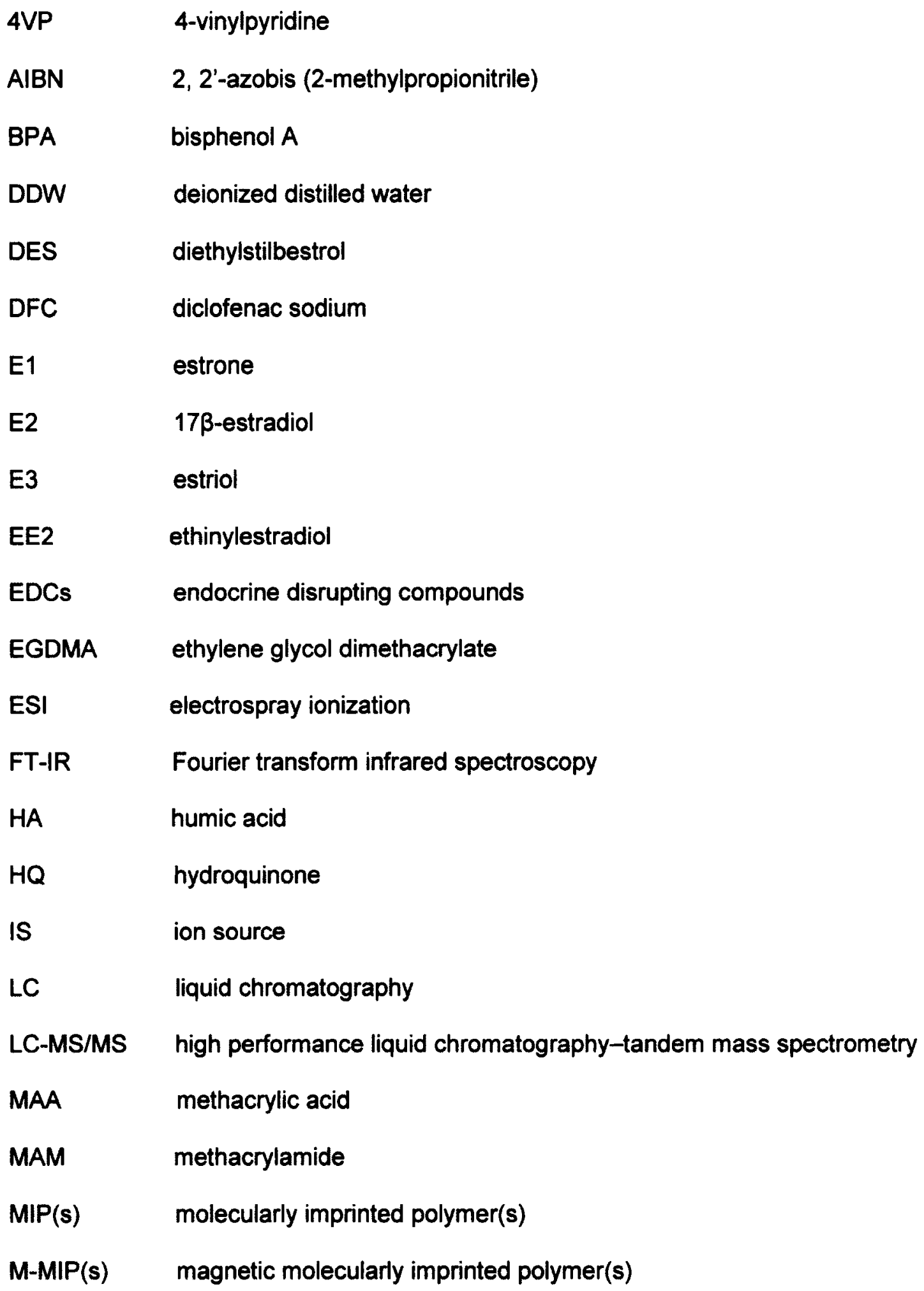




$\begin{array}{ll}\text { M-NIP(s) } & \text { magnetic non-imprinted polymer(s) } \\ \text { MNPs } & \text { magnetic nanoparticles } \\ \text { MRM } & \text { multiple reaction monitoring } \\ \text { NIP(s) } & \text { non-imprinted polymer(s) } \\ \text { NTU } & \text { nephelometric turbidity units } \\ \text { OA } & \text { oleic acid } \\ \text { PES } & \text { polyethersulfone } \\ \text { PH } & \text { phenol } \\ \text { RW } & \text { river water } \\ \text { SDS } & \text { sodium dodecyl sulfate } \\ \text { SEM } & \text { scanning electron microscopy } \\ \text { SPE } & \text { solid-phase extraction } \\ \text { TW } & \text { tap water } \\ \text { UV-Vis } & \text { ultraviolet-visible }\end{array}$




\section{Chapter 1}

Introduction

\subsection{Research goal}

The main goal of this thesis is the development of magnetic molecularly imprinted polymer (M-MIP) for the removal of endocrine disrupting compounds (EDCs). Integrated with magnetic properties, this artificial receptor can be potentially used to adsorb EDCs and the adsorbent can be magnetically separated from the solution after treatment, allowing for a rapid, efficient and cost-effective removal of EDCs from aqueous environment.

\subsection{Scope of the work}

To reach this fundamental goal, four major research tasks are involved in this thesis, including:

- Development of molecularly imprinted polymer encapsulated magnetic nanoparticles;

- Comparison of binding efficiency of magnetic MIP to non-magnetic MIP;

- Determination of M-MIP recovery efficiency and comparing magnetic separation to conventional isolation approaches; and

- Determination of reuse and regeneration potential of the developed magnetic MIP.

In addition, the development of a sensitive analytic method is required to advance the EDCs research at trace $(\mathrm{ng} / \mathrm{mL})$ concentration levels. 


\subsection{Research methodology}

Molecular imprinting technique is incorporated with magnetic properties to synthesize MIP encapsulated M-MIP. MIP layer is formed on magnetic core using a noncovalent imprinting approach [1] through polymerizing an estrogen-monomer complex with a cross linker. Aqueous magnetic fluid (ferrofluid) [2,3] is designed through surface modification of magnetite particles with surfactants.

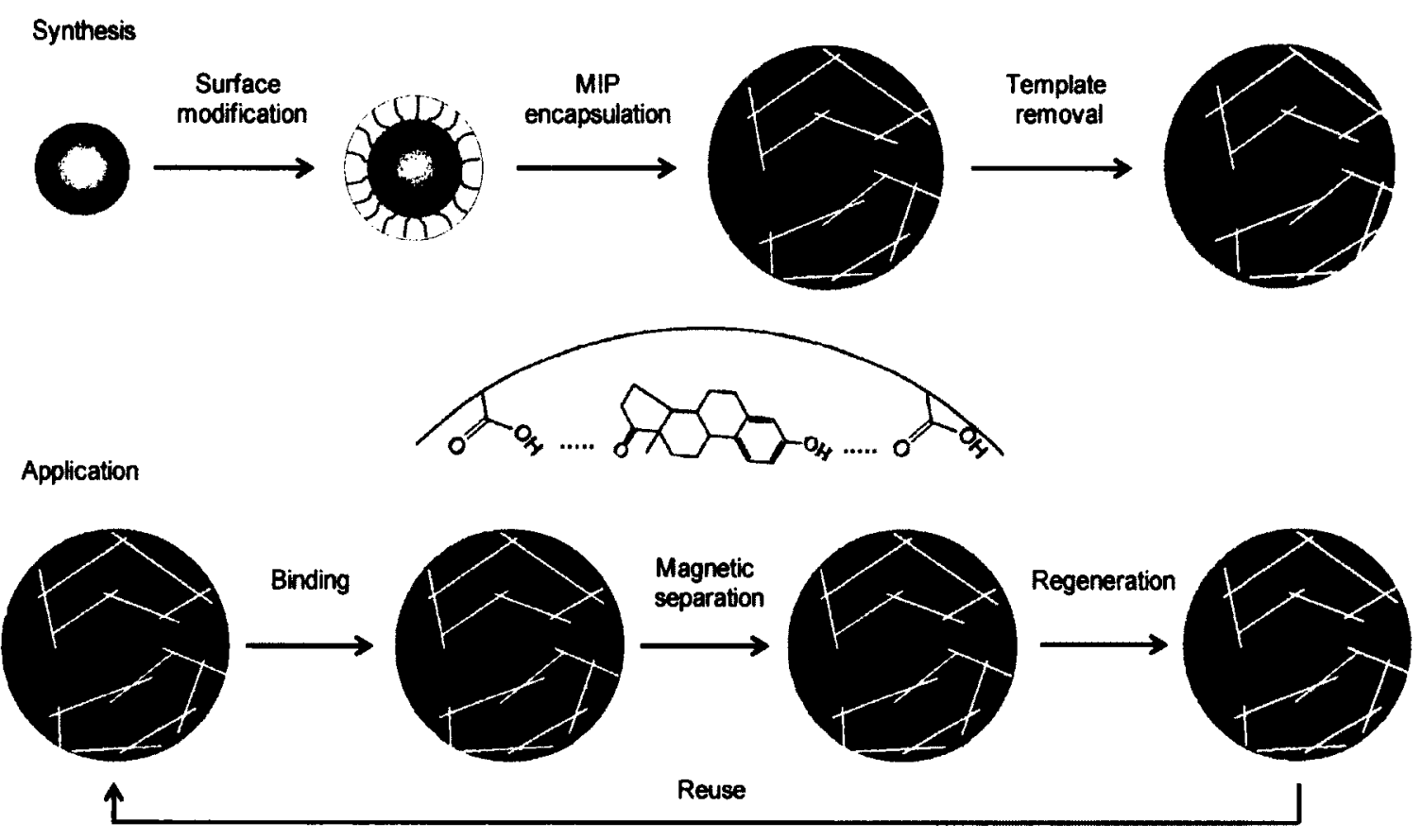

Fig. 1.1 Conceptual illustration of research scheme

A hydro-organic colloid suspension is adopted to create a compatible medium for an efficient coating process. A new strategy - ultrasonication assisted synthesis is developed to generate core-shell magnetic hybrid. Particle characteristic is investigated by spectroscopic techniques. High performance liquid chromatography-tandem mass spectrometry (LC-MS/MS) is used for quantifying biding performance at trace level. Binding characteristics and reusability are assessed toward the selected EDCs. The 
proposed protocol for the removal of EDCs is finally evaluated using spiked aqueous environment samples. A conceptual description of the methodology is illustrated in Fig.

\section{1.}

\subsection{Thesis organization}

This thesis is organized in a topic oriented structure as follows:

Chapter 2

E2 M-MIP

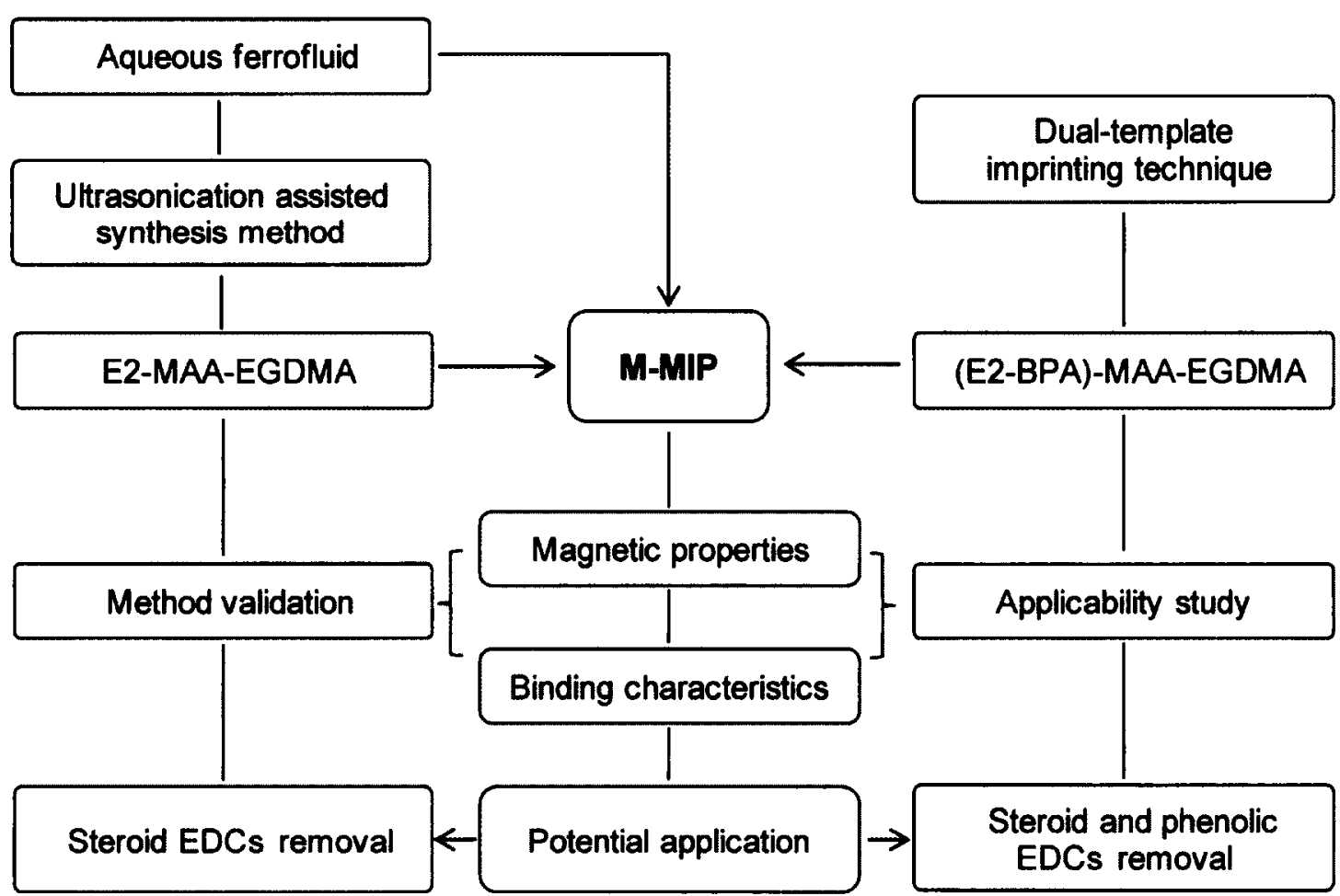

Fig. 1.2 Chapter structure

Chapter 2 of this thesis provides a brief introduction to the molecularly imprinting techniques and synthetic routes of magnetic nanoparticles (MNPs), with a focus on the synthesis and characterization of $17 \beta$-estradiol (E2) imprinted magnetic MIP. A newly 
developed method - ultrasonication assisted synthesis is described in detail. The binding characteristics and adsorption capacity of M-MIP are investigated and compared to the non-magnetic MIP formulated in conventional synthetic routes. Magnetic transferability and kinetic property are studied for a satisfactory M-MIP recovery in the presence of a magnetic field. Chapter 2 proposes a potential application of M-MIP for the removal of E2 and the structurally related steroid EDCs.

Chapter 3 describes a method using dual-template imprinting strategy in an attempt to remove steroid and phenolic EDCs simultaneously. The main parameters that affect M-MIP synthesis are discussed. The effect of environmental variables on adsorption capacity and the reusability of M-MIP are investigated. Magnetic separation efficiency is studied and compared to that of centrifugation and membrane filtration. The binding characteristics are examined with Freundlich isotherm model and particle surface heterogeneity is identified. In addition, the practicability and applicability of classspecific removal of EDCs by E2-BPA imprinted MIP are verified using spiked aqueous environmental samples.

Chapter 4 briefly concludes the current research work on the magnetic MIP and suggests some improvements in future research.

\subsection{EDCs imprinted M-MIPs as selective adsorbents}

Molecular imprinting technique is an attractive method for the generation of polymeric particles that have high selectivity toward a given target or a group of target molecules $[4,5]$. M-MIPs are the magnetic carriers with various chemical functionalities on the surface [6]. Rapid, affordable and selective removal of target pollutants can be attained by making use of magnetic response of MNPs and tailor-made selectivity of MIPs [7]. 
Surface functionalization and modification of MNPs to introduce functionalities for selective binding of EDCs have been pursued in various synthetic procedures. A recent research demonstrated a semi-covalent imprinting strategy for synthesizing estrone (E1) imprinted magnetic polymer [8]. In this protocol, template-monomer complex was formed by the reaction of E1 with 3-(triethoxysilyl)propyl isocyanate. The template was linked to the silica coated magnetic core via a thermally reversible bond. The postsynthesis template removal was simply achieved by a thermal reaction, leaving recognition sites on the surface of silica shell. In comparison with traditional MIPs, E1 MMIP exhibited an easy and quick target access to the imprinted sites.

A general procedure of preparing surface-imprinted magnetic fluorescence beads via reversible addition-fragmentation chain transfer polymerization was developed with E2 template [9]. This polymerization approach allows the tailoring of macro-molecules with predetermined molecular weight, terminal functionality and narrow molecular weight distribution. The M-MIP nanosphere was designed in a double-layer structure consisting of an inner layer of fluorescein isothiocyanate and an outer layer of MIP. Binding results exhibited an appreciable uptake of E2 over structurally related compounds. The combination of magnetization, fluorescence labeling and molecular imprinting makes the M-MIP applicable of selective binding and detection of non-fluorescent EDCs and facilitating a convenient magnetic separation.

A surface molecular self-assembly strategy for molecular imprinting on MNPs was explored [10]. $y$-methacryloxypropyltrimethoxysilane was assembled on the surface of a silica-modified magnetic core through free radical polymerization, followed by a copolymerization to assemble E2-methacrylic acid-ethylene glycol dimethacrylate MIP. In this way, majority of the recognition sites could be generated on the particle surface, reducing diffusion resistance for an efficient rebinding. The approach suggested a potential for fast enrichment and extraction of estrogens from complicated matrices. 
Meanwhile, M-MIPs with phenolic endocrine disruptors as templates were synthesized and studied for binding characteristics. A bisphenol A (BPA) M-MIP was prepared by mini-emulsion polymerization [11]. Comparative studies on the adsorption of BPA and the analogue diethylstilbestrol (DES) displayed a favorable uptake of the template. A solid-phase extraction (SPE) procedure, assisted by magnetic separation, was then developed to extract BPA from environmental water.

Bisphenol F (BPF) was reported as a dummy template to prepare M-MIP as an adsorbent in SPE for determining BPA from real water samples [12]. Binding tests showed that BPF M-MIP had high selectivity to BPA with a range of recoveries from around $84 \%$ to $94 \%$.

A BPA M-MIP was synthesized via surface initiated atom transfer radical polymerization (ATRP) [13]. The magnetic core was encapsulated with a polychloromethylstyrene layer through mini-emulsion polymerization. The M-MIP had a saturation magnetization of $25.2 \mathrm{emu} \mathrm{g}^{-1}$ and satisfactory response to a magnetic field at acidic and alkaline conditions. The M-MIP possessed higher affinity toward BPA than structurally related analogues. Similar studies with ATRP approach supported the findings with BPA M-MIP as highly selective sorbents in SPE for extracting trace BPA, in which lower binding capability of BPA M-MIP toward 4- $n$-octylphenol and bisphenol A diglycidyl ether was observed [14], suggesting the potential of ATRP in EDCs imprinting for the preparation of M-MIPs.

Up to date, the researches in the preparation of EDCs imprinted M-MIPs are limited to explore the synthetic routes with less focus on their application. The magnetic properties of M-MIPs were more characterized for magnetic response ability rather than the development of practical separation procedures. However, the progress that is made in this area provides a solid basis for this thesis research. 


\section{References}

1. L. Chen, S. Xu, J. Li, Chem. Soc. Rev., 40, 2922 (2011).

2. M. Faraji, Y. Yamini, and M. Rezaee, J. Iran. Chem. Soc., 7, 1 (2010).

3. P. Berger, N.B. Adelman, K.J. Beckman, D.J. Campbell, A.B. Ellis, G.C. Lisensky, J. Chem. Educ., 76, 943 (1999).

4. G. Wulff, Angew. Chem. Int. Ed. 34, 1812 (1995).

5. Y. Watabe, T. Kubo, T. Nishikawa, T. Fujita, K. Kaya, K. Hosoya, J. Chromatogr. A, 1120, 252 (2006).

6. G. Guan, B. Liu, Z. Wang, Z. Zhang, Sensors, 8, 8291 (2008).

7. M. Faraji, Y. Yamini, M. Rezaee, J. Iran. Chem. Soc., 7, 1 (2010).

8. X. Wang, L. Wang, X. He, Y. Zhang, L. Chen, Talanta, 78, 327 (2009).

9. Y. Li, C. Dong, J. Chu, J. Qi, X. Li, Nanoscale, 3, 280 (2011).

10. S. Wang, Y. Li, M. Ding, X. Wu, J. Xu, R. Wang, T. Wen, W. Huang, P. Zhou, K. Ma, X. Zhou, S. Du, J. Chrom. B, 879, 2595 (2011).

11. Y. Ji, J. Yin, Z. Xu, C. Zhao, H. Huang, H. Zhang, C. Wang, Anal. Bioanal. Chem., 395, 1125 (2009).

12. Z. Lin, W. Cheng, Y. Li, Z. Liu, X. Chen, C. Huang, Anal. Chim. Acta, 720, 71 (2012).

13. J. Liu, W. Wang, Y. Xie, Y. Huang, Y. Liu, X. Liu, R. Zhao, G. Liu, Y. Chen, J. Mater.Chem., 21, 9232 (2011).

14. Z. Xu, L. Ding, Y. Long, L. Xu, L. Wang, C. Xu, Anal. Methods, 3, 1737 (2011). 


\section{Chapter 2}

Ultrasonication Assisted Synthesis of MIP Encapsulated Magnetic Nanoparticles for Rapid and Selective Removal of $17 \beta$-Estradiol from Aqueous Environment

\subsection{Introduction}

Low-doses of endocrine disrupting compounds (EDCs), pharmaceuticals and personal care products in water have been reported $[1,2]$. There is growing concern about the ecological and human health impact of these emerging compounds. EDCs can have adverse effects on the human endocrine system at trace concentration levels, and pose a disproportionate threat to fetal development. Their effects on aquatic organisms have been documented both in the lab and in the field, and the feminization of male fish has been linked to wastewater treatment plant discharges [3].

Conventional pollution control technologies are limited by poor performance and high costs of existing sorbent materials. Hence, considerable research is directed toward the development of new types of sorbents [4]. The past few decades have seen enormous progress in molecular imprinting techniques, including the development of polymer design protocols and their compatibility for use in aqueous environment. Molecularly imprinted polymers (MIPs) have been employed in an increasing number of applications where molecular binding events are of interest. The good stability and low cost of MIPs make them advantageous for use in industrial scale production $[5,6]$. MIPs are attracting more interest in environmental, food and pharmaceutical applications for the extraction or clean-up of different classes of compounds from various complex matrices. However, in the use of MIP particles as sorbent for the removal of micropollutants such as EDCs, the separation of polymer particles from aqueous medium appears to be a challenging issue from the engineering perspective. 
In recent years, magnetic nanoparticles (MNPs) have received considerable attention due to their specific magnetic properties [7]. The environmental applications of MNPs are particularly significant. There is growing interest to combine MIPs with MNPs, based on the formation of magnetic responsive core-shell hybrids. Rapid, affordable and selective removal of target contaminants can be attained by making use of magnetic response of MNPs and tailor-made selectivity of MIPs.

Among the estrogen imprinted MIPs, 17ß-estradiol (E2) has been templated to develop MIPs as synthetic estrogen receptors [8-11]. 17a-estradiol (a natural epimer of E2) [12] and 6-ketoecradiol (a pseudo E2) [13] were reported for MIPs preparation to target E2. The researches had specific focus on optimizing the formulation of MIPs, including choice of monomers, cross-linkers and porogens. The establishment of optimal ratio among the polymerization components and rational design of synthetic routes were of interest for the desired morphology, porosity, cavity distribution and accessibility. In more recent works, inorganic materials - silica [14] and silica-modified magnetic nanoparticles [15] were introduced for generating core-shell polymeric particles for surface imprinting to enhance rebinding performance. New synthetic technique such as microwave irradiation has been applied to reduce polymerization time and improve imprinting efficiency [16]. In brief, E2 imprinted polymers have been attempted as an attractive alternative to the conventional stationary phase for separation and determination in high performance liquid chromatography and solid phase extraction, and a class of promising sorbents for purification, enrichment and separation. Highly efficient removal of E2 from water was achieved using macro-porous cryogel adsorption medium with embedded MIP particles [17] and the combination of liquid membranes and MIP in the extraction of E2 from aqueous samples [18]. The effect of environmental factors such as humic acid and $\mathrm{pH}$ on the adsorption of estrogenic compounds by E2 imprinted MIPs were examined in water and wastewater samples [19-21]. The 
adsorption kinetics of MIP particles in aqueous medium was also investigated [22].

For potential environmental applications, MIP particles with captured estrogens must be removed from aqueous matrix after treatment. Ideally this can be achieved in a simple, quick and energy-saving manner. If magnetic materials can be incorporated into the imprinted sorbents, magnetic separation can be performed with applied magnetic field. Consequently, a rapid and selective purification and separation process can be enabled. This approach has specific advantages for estrogenic effect reduction from water streams. Magnetic separation has its advantages over conventional separation methods such as coagulation and flocculation in which the addition of chemicals are often required. It is also a preferred technique over filtration in a cost-effective way. The regeneration and reuse of MIP particles [23] will be facilitated. In fact, magnetic separation has been widely used for in-lab template removal and clean-up step instead of centrifugation. The application of magnetic MIPs for removal of estrone [24] and bisphenol A [25] from water samples has been tested in lab scale.

In this work, MIP/NIP-encapsulated MNPs (M-MIP/M-NIP) were prepared using ultrasonication assisted synthesis in a hydro-organic polymerization medium. The resultant MIP particles were characterized with respect to their structure, sizes and size distribution. The recovery of magnetic MIP in the presence of a magnetic field was determined in a quantitative manner. Molecular recognition and removal efficiency of $M$ MIP toward estrogenic compound, as represented by E2, was compared to MIPs formulated in organic media in conventional synthetic routes. The proposed protocol for removal of estrogenic compounds from aqueous environment was verified using tap water samples spiked with E2. 


\subsection{Experimental}

\subsubsection{Materials}

Oleic acid $(\mathrm{OA})$ and iron (II) chloride tetrahydrate $\left(\mathrm{FeCl}_{2} \cdot 4 \mathrm{H}_{2} \mathrm{O}\right)$ were purchased from Sigma-Aldrich (Oakville, ON, Canada), sodium dodecyl sulfate (SDS) was obtained from Pierce (Rockford, IL, USA). 17ß-estradiol (E2), bisphenol A (BPA), methacrylic acid (MAA) and ethylene glycol dimethacrylate (EGDMA) were purchased from Aldrich (St. Louis, MO, USA). 2, 2'-azobis (2-methylpropionitrile) (AIBN) was purchased from Pfaltz \& Bauer (Waterbury, CT, USA). Iron (III) chloride anhydrous $\left(\mathrm{FeCl}_{3}\right)$ was obtained from Riedel-de Haen (Seelze, Germany). Ammonium hydroxide was supplied by Fisher Scientific (Pittsburgh, PA, USA). All chemicals and reagents were used as purchased without further purification.

The stock standard solutions of E2 and BPA at $1000 \mu \mathrm{g} / \mathrm{ml}$ were prepared in methanol and stored at $-20^{\circ} \mathrm{C}$. Individual standard solutions were prepared at different concentrations in $50 \%$ water-methanol diluted from stock solutions.

\subsubsection{Preparation of MNPs}

Chemical precipitation was used to synthesize $\mathrm{Fe}_{3} \mathrm{O}_{4}$ MNPs by mixing $\mathrm{Fe}$ (II) and $\mathrm{Fe}$ (III) chlorides under alkaline condition and inert atmosphere. $\mathrm{FeCl}_{3}(2.54 \mathrm{~g})$ and $\mathrm{FeCl}_{2} \cdot 4 \mathrm{H}_{2} \mathrm{O}(1.73 \mathrm{~g})$ was dissolved in $80 \mathrm{~mL}$ of deionized distilled water (DDW) contained in a three-neck flask with vigorous stirring under helium. $10 \mathrm{~mL}$ of ammonium hydroxide was added dropwise after temperature reached $80^{\circ} \mathrm{C}$. The resultant $\mathrm{Fe}_{3} \mathrm{O}_{4}$ was maintained for $30 \mathrm{~min}$ at $80{ }^{\circ} \mathrm{C}$ after the addition of ammonium hydroxide for aging and enhanced magnetization. MNPs precipitate was collected by applying an external magnetic field and dried in the vacuum after DDW washing. 


\subsubsection{Preparation of aqueous ferrofluid}

Aqueous ferrofluid was prepared through surface modification of $\mathrm{Fe}_{3} \mathrm{O}_{4}$ MNPs with $\mathrm{OA}$ and SDS surfactants. $200 \mathrm{mg}$ of $\mathrm{Fe}_{3} \mathrm{O}_{4}$ was dissolved in a volumetric flask containing $20 \mathrm{~mL}$ of DDW and sonicated for $5 \mathrm{~min}$ at $60^{\circ} \mathrm{C}$. A total of $139 \mu \mathrm{L} \mathrm{OA}$ was added in three equal volumes, followed by $5 \mathrm{~min}$ sonication after each addition. $20 \mathrm{~mL}$ of DDW containing $134 \mathrm{mg}$ of SDS was introduced. This was followed by $10 \mathrm{~min}$ ultrasonication at ambient temperature. The prepared $\mathrm{Fe}_{3} \mathrm{O}_{4}-\mathrm{OA}-\mathrm{SDS}$ aqueous ferrofluid was at 5.0 $\mathrm{mg} / \mathrm{mL}$ (in $\mathrm{Fe}_{3} \mathrm{O}_{4}$ ).

\subsubsection{Preparation of pre-polymerization solution}

The pre-polymerization solution was prepared in organic solvent with polymer material composition referring to a reported method [11]. The template, $68.1 \mathrm{mg}$ of E2, was first added into $10 \mathrm{~mL}$ of acetone/acetonitrile $(1: 3, \mathrm{v} / \mathrm{v})$. The functional monomer, $172 \mu \mathrm{L}$ of MAA, was then added and well mixed. The mixture was put in the dark for $2 \mathrm{~h}$ to allow for hydrogen bonding. The cross-linker, $317 \mu \mathrm{L}$ of EGDMA, was introduced shortly before final polymerization.

\subsubsection{Synthesis of M-MIP}

The encapsulation of MNPs with MIP was performed using ultrasonication assisted synthesis in a ferrofluid and pre-polymerization solution medium (10:10, v/v). $10 \mathrm{~mL}$ of aqueous ferrofluid was suspended into $10 \mathrm{~mL}$ of pre-polymerization solution, followed by $1 \mathrm{~min}$ of sonication degassing. The initiator, $11.5 \mathrm{mg}$ of AIBN, was then added. This was followed by 7 min purging with nitrogen for deoxygenating. The reaction vial was capped and sealed firmly with parafilm. The vial was mounted with self-assembled holder and placed in an ultrasonication bath (Bransonic ultrasonicator, 2510R-DTH, $100 \mathrm{~W}, 42 \mathrm{KHz}$, USA) at $65^{\circ} \mathrm{C}$ for $2 \mathrm{~h}$ polymerization. The resultant particles were separated by an 
externally applied magnetic field and followed by intensive template extraction with acetic acid/methanol (10:90, v/v). DDW and acetonitrile washing were performed before vacuum drying. As a reference, non-imprinted polymer encapsulated MNPs were prepared in the absence of template using the same synthetic route.

\subsubsection{SEM and FT-IR scans}

The morphology and structure of M-MIP and M-NIP were examined by scanning electron microscopy (SEM) (Tescan-Vega II-XMU, Czech). Fourier transform infrared spectroscopy (FT-IR) analysis was carried out on a FT-IR spectrometer (Varian 1000 FT-IR, USA). $2.0 \mathrm{mg}$ of sample powder was pulverized and mixed with $20 \mathrm{mg}$ of $\mathrm{KBr}$. The mixture was dried and pressed to form pellets for recording FT-IR spectrum.

\subsubsection{Magnetic transferability}

Magnetic transferability was examined on an Abraxis 60-tube magnetic separator and Ultraviolet-Visible (UV-Vis) spectrophotometer (Varian Cary 3, USA). Stock solutions were prepared by dispersing $1.0 \mathrm{mg}$ of sample (MNPs, M-MIP and M-NIP) in $10 \mathrm{~mL}$ of DDW with 10 min sonication, from which a series of working solutions ranging from 0.001 to $0.1 \mathrm{mg} / \mathrm{mL}$ were prepared. $2.0 \mathrm{~mL}$ of the standard solutions were transferred to UV-Vis cells for absorbance measurement. The wavelength was set at $380 \mathrm{~nm}$ for setting up calibration curves. $1.0 \mathrm{~mL}$ of $0.1 \mathrm{mg} / \mathrm{mL}$ sample was transferred to test tube and placed in magnetic separator for a period ranging from 0.25 to $30 \mathrm{~min}$. The amount of remained particles in solution was measured by UV-Vis absorbance. All samples were run in duplicates.

\subsubsection{Optimization of LC-MS/MS}

Analyses were conducted on an AB Sciex API 2000 triple quadrupole tandem 
mass spectrometer coupled with a Shimadzu Prominence liquid chromatograph using an analytical column (Phenomenex, $50 \times 2.00 \mathrm{~mm}, 4$ micron, USA) at ambient temperature. The flow rate was set at $0.2 \mathrm{~mL} / \mathrm{min}$, using water/acetonitrile mobile phase $(30: 70, \mathrm{v} / \mathrm{v})$ containing $0.1 \%$ ammonia. The mass spectrometry detection was performed using Electrospray lonization (ESI) probe in negative polarity. The source temperature was set at $350{ }^{\circ} \mathrm{C}$. The main MS/MS parameters were optimized by direct infusion of $100 \mathrm{ng} / \mathrm{mL}$ standard analytes dissolved in water/acetonitrile containing $0.1 \%$ ammonia.

\subsubsection{Adsorption performance}

M-MIP adsorption performance was assessed by suspending a known amount of particles in aqueous solution containing E2 for incubation. After magnetic separation, the supernatant was sampled for LC-MS/MS analysis to determine the residual concentration. M-NIP was assessed similarly to compare the binding efficiency. $1.0 \mathrm{mg}$ of M-MIP was suspended into $2.0 \mathrm{~mL}$ of DDW containing $0.5 \mu \mathrm{g} / \mathrm{mL}$ of E2. The solution was then sonicated for $10 \mathrm{~min}$ to disperse the particles, followed by $30 \mathrm{~min}$ shaking. The mixture was evenly transferred into two glass test tubes and placed on magnetic separator. After 30 min of magnetic separation, the supernatant was sampled for LCMS/MS measurement. M-NIP was treated in the same way. Similarly, $1.0 \mathrm{mg}$ of M-MIP was suspended into $2.0 \mathrm{~mL}$ of DDW containing $0.5 \mu \mathrm{g} / \mathrm{mL}$ of BPA for comparison study.

\subsection{Results and discussion}

\subsubsection{Synthesis of MIP encapsulated MNPs}

\subsubsection{Preparation of aqueous ferrofluid}

Fig. 2.1 presents the aqueous ferrofluid prepared from MNPs and surfactants. OA was used as the primary surfactant and SDS as the secondary surfactant for surface 
modification to create a colloidal suspension. It is known that MNPs tend to form clusters to reduce surface energy. The surfactants work as dispersion agents by adhering to MNPs and creating a net repulsion, raising the energy required for MNPs to agglomerate, and stabilizing the colloid [26].

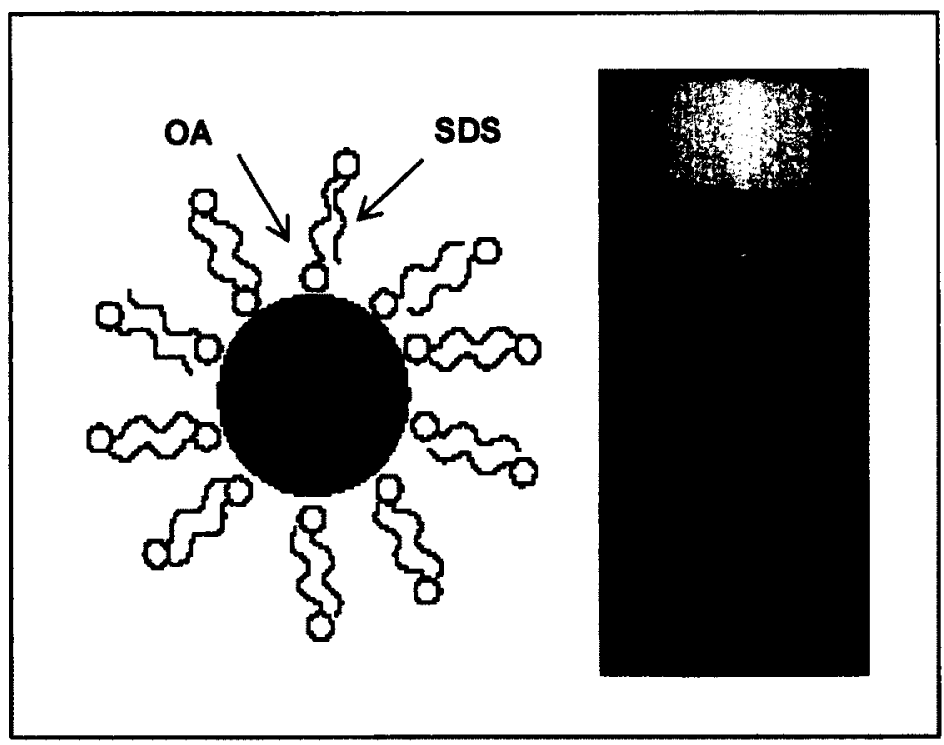

Fig. 2.1 Aqueous ferrofluid and dual-layer structure illustration

The addition of surfactants OA and SDS, in assistance with ultrasonication, provides an effective means of breaking up the agglomerate structure in aqueous suspension through the change in surface property of $\mathrm{Fe}_{3} \mathrm{O}_{4}$ MNPs. As amphiphilic surfactant, $\mathrm{OA}$ possesses strong binding capability toward $\mathrm{Fe}_{3} \mathrm{O}_{4}$ through the carboxyl group. In the weakly acidified solution at $\mathrm{pH}$ slightly over 5.0 , positively charged $\mathrm{Fe}_{3} \mathrm{O}_{4}$ particles attracted OA to form a layer with outward chains. With sonication assistance, OA was able to be dispersed in water and coated on MNPs at $60^{\circ} \mathrm{C}$. However, the resulting $\mathrm{Fe}_{3} \mathrm{O}_{4}-\mathrm{OA}$ exhibited a poor dispersion in aqueous phase attributed to the hydrophobic surface of $\mathrm{Fe}_{3} \mathrm{O}_{4}-\mathrm{OA}$. The dispersion worsened with increasing amount of OA. Introduction of SDS significantly improved the suspension of OA-coated particles. 
SDS consists of a 12-carbon tail attached to a sulfate group. Addition of SDS resulted in a hydrophobic-hydrophilic change by the formation of a SDS outer layer through interactions between the alkyl chains of SDS and the unsaturated OA chains [27]. An examination of various OASDS molar ratios, ranging from1:0.1 to $1: 1.5$, revealed the solubilizing action of SDS on poorly dispersed $\mathrm{Fe}_{3} \mathrm{O}_{4}-\mathrm{OA}$. At higher OASDS ratios (>1: 0.75), aggregation was observed. Aggregation increased with decreased SDS content. At a molar ratio of $1: 1$, an evenly dispersed $\mathrm{Fe}_{3} \mathrm{O}_{4}-\mathrm{OA}-\mathrm{SDS}$ magnetic fluid was realized with the formation of surfactant modified dual-layer. In this way, $5.0 \mathrm{mg} / \mathrm{mL}$ of $\mathrm{Fe}_{3} \mathrm{O}_{4}$ was dispersed in water with subsequent introduction of OA and SDS at a final concentration of $12.5 \mathrm{mM}$ for each under sonication assistance.

\subsubsection{Polymerization in the presence of water}

E2 imprinted MIP was originally prepared using MAA-EGDMA in an organic mixture of acetone and acetonitrile $(1: 3, v / v)[11]$. To enable the encapsulation of MIP on MNPs with aqueous ferrofluid, the introduction of water was examined in a water bath without MNPs to investigate the feasibility of polymerization in the presence of water.

The effect of initiator was first examined by adding various amounts of AIBN, from $1 \%$ to $10 \%$ (in wt. $\%$ relative to MAA), into a polymerization solution containing $50 \%$ water. In an $18 \mathrm{~h}$ cycle, MIP particles were formed at all initiator levels above $2 \%$, but $1 \%$ initiator was not sufficient to initiate the polymerization. Formation of nuclei during nucleation stage responded to the increasing initiator concentration although it was not linearly proportional to the initiator concentration owing to the complexity of free radical polymerization. Compared to the polymerization in organic solvents, MIP particles formation rate was accelerated. More rapid diffusion and effectiveness in reducing the activation energy of the reaction between radical and polymerization components in water may explain the acceleration [28]. At $6.5 \%$ of initiator, a range of temperatures, $60-$ 
$70{ }^{\circ} \mathrm{C}$, appeared to reach the same $\%$ conversion but at different formation rates. It showed that polymerization time strongly depends on temperature, as previously reported [29]. It was noted that E2-MAA-EGDMA polymerization in the presence of water resulted in the formation of a jelly-like mixture. Addition of surfactants (OA and SDS) or application of magnetic stirring did not provide satisfactory solution. After dryness in vacuum, white flakes were produced and further grinding was required. It suggests the complexity of polymerization with high aqueous content.

\subsubsection{Ultrasonication assisted synthesis}

Ultrasonication was found to be effective in eliminating the formation of jelly-like mixture. It was further employed to develop a synthetic route for the preparation of coreshell E2 imprinted magnetic MIP, as illustrated in Fig. 2.2. The ultrasound enhanced the polymerization rate and spherical nanoparticles [30]. Ultrasonication caused an increase in the number of free radicals and resulted in the formation of more nuclei, thus facilitating MIP growth around MNPs seed.

To minimize the formation of core-free MIP nuclei, the optimal ratio between seed MNPs and MIP was established by changing MNPs concentration from 2.0 to 10.0 $\mathrm{mg} / \mathrm{mL}$ in the polymerization mixture. At concentrations $<4.0 \mathrm{mg} / \mathrm{mL}$, many core-free MIP particles were produced, as confirmed by checking their magnetic response. At concentrations $>6.0 \mathrm{mg} / \mathrm{mL}$, un-encapsulated magnetic particles were abundant. The amount of seed MNPs was optimized at $5.0 \mathrm{mg} / \mathrm{mL}$. The aqueous content had significant effect on MIP encapsulation. At a high aqueous content (60\%), polymerization slowed down due to the dilution of the polymerization components. At a low aqueous content (40\%), a mixture of un-encapsulated MNPs, core-free MIP particles and magnetic MIP

particles was produced. A $50 \%$ aqueous content containing $50 \mathrm{mg}$ MNPs enabled the formation of fully MIP-encapsulated MNPs in a 2-h ultrasonication assisted synthesis. 
The encapsulation of NIP produced similar findings but showed a quick nucleation and particle growth than MIP, indicated by the quicker appearance of cloud-like particles in the NIP polymerization solution.

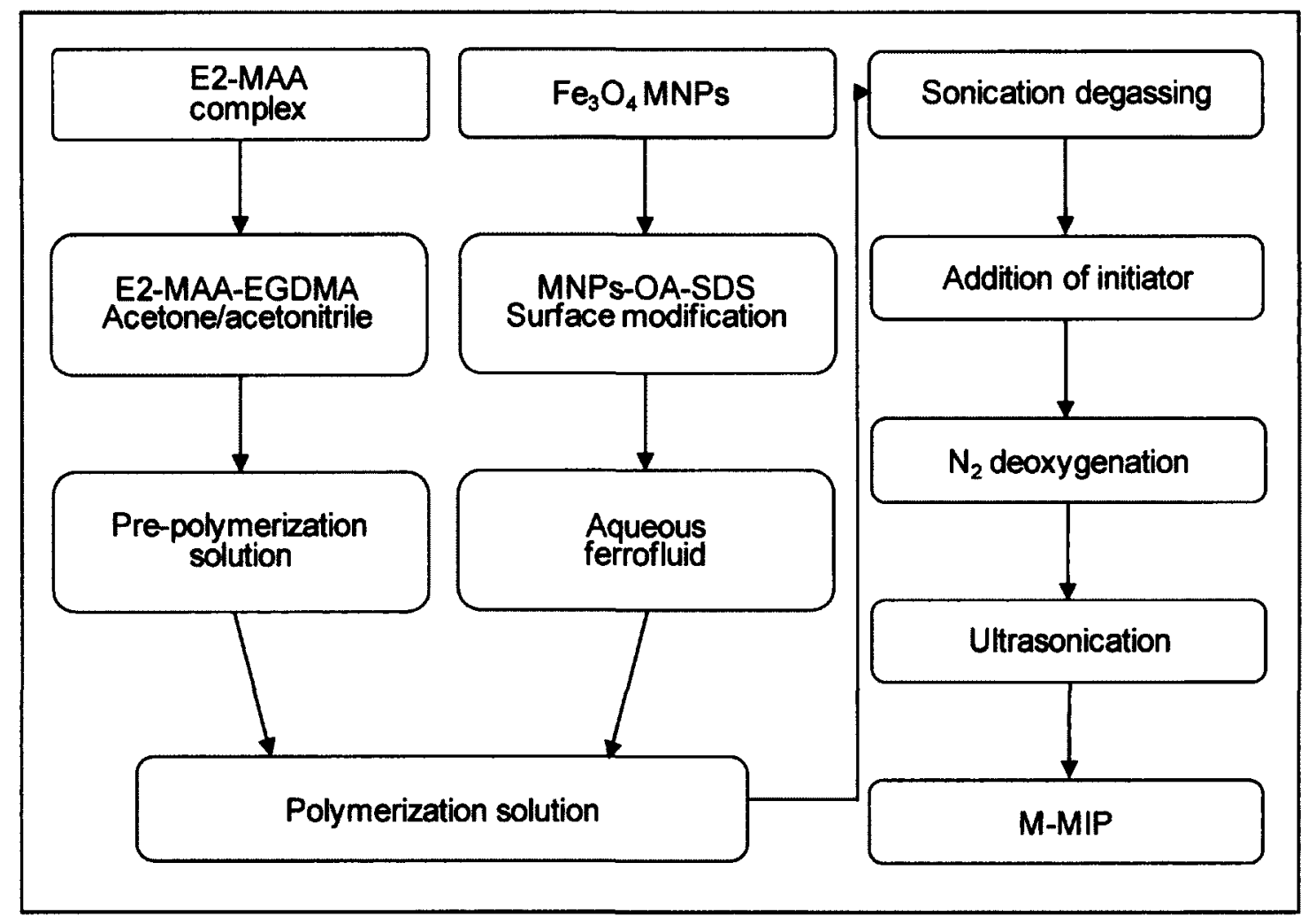

Fig. 2.2 Ultrasonication assisted synthesis of M-MIP

In comparison to the conventional procedures for preparing MIPs, the polymerization duration with ultrasonication assisted synthesis was reduced to $2 \mathrm{~h}$. With proper deoxygenating, purging with inert gas was not required through the course of polymerization. Most importantly, ultrasonication provides a powerful means to evenly disperse magnetic seeds in the polymerization medium, replacing mechanical stirring that is usually required by the conventional methods and microwave irradiation techniques $[31,32]$.

Nanoscale $\mathrm{Fe}_{3} \mathrm{O}_{4}$ MNPs and M-MIP particles tend to be agglomerated and 
settled down from solution during polymerization course. Effective means of deagglomerating and dispersing are required. The employment of ultrasonication produces a more homogenous mixture by increasing the solubility of the components [33]. Ultrasonication is widely used in the analytical laboratories for its versatile effects to improve, accelerate sample preparation [34]. Ultrasonication improves the mixing of magnetic particles and increases the mass-transfer at the particle surface, leading to smaller particle size and higher uniformity.

\subsubsection{Characterization}

\subsubsection{Particle size and morphology}

A study on the physical features of the formulated particles can produce information important to the characteristics of rebinding. Fig. 2.3 displays the SEM scans of MNPs, MNPs-OA-SDS, M-NIP and M-MIP. It can be seen from Fig. 3a that MNPs have an average diameter of $20 \mathrm{~nm}$. Fig. 2.3b shows the sizes of OA-SDS modified MNPs, ranging from 26 to $28 \mathrm{~nm}$. Surface modification did not cause a significant increase in size. An average thickness of 3-4 $\mathrm{nm}$ suggests the formation of an OA-SDS surfactants layer. As shown in Fig. $2.3 \mathrm{c}$ and 2.3d, the average size of M-NIP and M-MIP is at 200 and $300 \mathrm{~nm}$, respectively. This reveals that there is an increase in diameter by $100 \mathrm{~nm}$ from M-NIP to M-MIP. This could be attributed to the existence of templateshaped cavities created through hydrogen bonding during polymerization. 


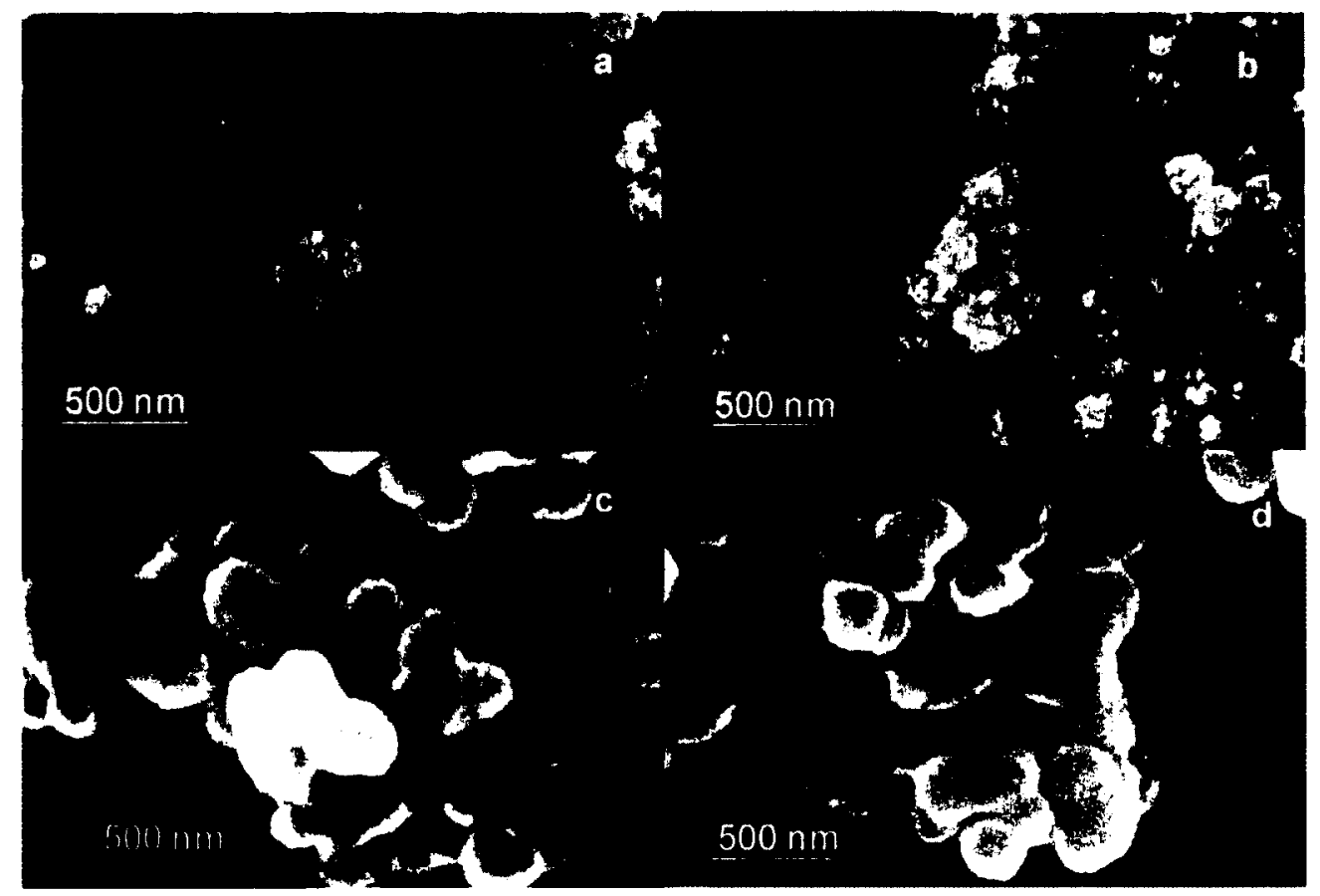

Fig. 2.3 SEM scans of MNPs (a), MNPs-OA-SDS (b), M-NIP (c) and M-MIP (d)

Fig. 2.3 clearly demonstrates the spherical morphology of M-MIP and M-NIP. The formulated particles exhibit fine sizes and narrow size distribution. The employment of ultrasonication dramatically increases the uniformity of the resulting particles, and facilitates the polymerization around the magnetic seeds, leading to a steady growth to the finished M-MIP and M-NIP. It is possible that ultrasonication also promotes an even binding sites population distribution.

\subsubsection{FT-IR spectra}

Fig. 2.4a shows the FT-IR spectrum of $\mathrm{Fe}_{3} \mathrm{O}_{4}$ nanoparticles. The bands in the FTIR spectrum of $\mathrm{Fe}_{3} \mathrm{O}_{4}$ at $586 \mathrm{~cm}^{-1}$ corresponds to the $\mathrm{Fe}-\mathrm{O}$ bond of $\mathrm{Fe}_{3} \mathrm{O}_{4}$. No other peaks with significant intensity were found. After $\mathrm{OA}$ coating, $\mathrm{Fe}-\mathrm{O}$ peak of $\mathrm{Fe}_{3} \mathrm{O}_{4}$ red shifted to $561 \mathrm{~cm}^{-1}$ (Fig. 2.4b). The vibrations at $2924 \mathrm{~cm}^{-1}$ and $2854 \mathrm{~cm}^{-1}$ are attributed 


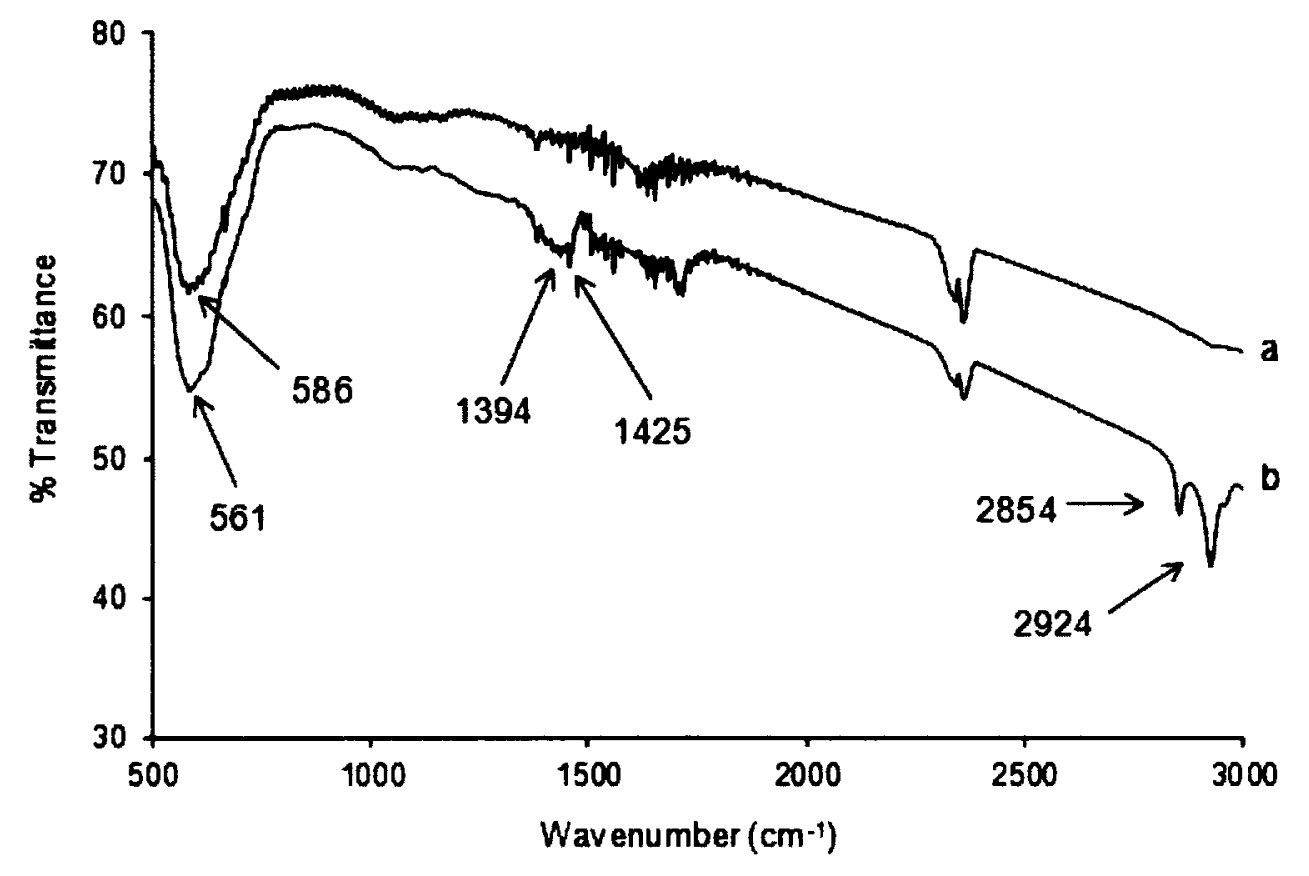

Fig. 2.4 FT-IR spectrum of MNPs (a) and MNPs-OA-SDS (b)

to the alkyl chains. The peak $1425 \mathrm{~cm}^{-1}$, resulting from the carboxylate unit vibration, suggests that $O A$ is bound through the carboxylate anion. The peak at $1394 \mathrm{~cm}^{-1}$ and weak band around $1045 \mathrm{~cm}^{-1}$ are the $\mathrm{C}-\mathrm{H}$ and $\mathrm{S}=0$ vibration peaks respectively, representing the SDS presence on the OA modified MNPs surface. The FT-IR spectrum of M-MIP (Fig. 2.5) reveals the nature of the bond formed between MNPs and MIP. The band at $1697 \mathrm{~cm}^{-1}$ indicates $C=0$ stretch vibration of $M A A$, and $1637 \mathrm{~cm}^{-1}$ the $C=C$ bond of MAA and EGDMA. The peak at $1729 \mathrm{~cm}^{-1}$ is attributed to the carboxylate vibration modes of EGDMA. These peaks show the presence of functional groups of monomer and cross linker in the synthesized M-MIP. 


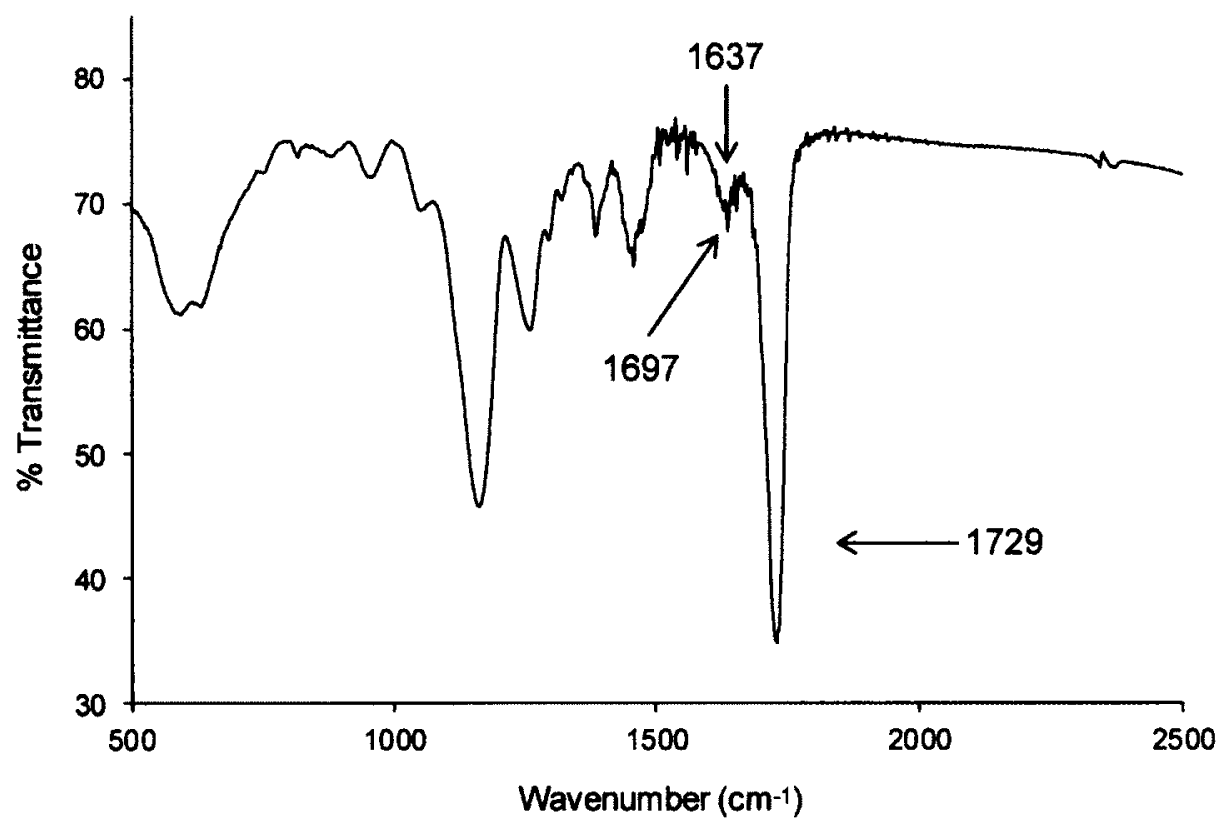

Fig. 2.5 FT-IR spectrum of M-MIP

\subsubsection{Magnetic transferability}

Vibrating sample magnetometry $[35,36]$ has been commonly employed to evaluate parameters of magnetic particles. In this work, a quantitative recycle of M-MIP and MNIP from aqueous medium on a timely basis was studied by combining magnetic separator and UV-Vis spectrometer to investigate magnetic response and magnetic transferability-the magnetic characterization of nanoparticles, defined as the susceptibility of superparamagnetic nanoparticles toward magnetic-field-assisted separation [37].

UV-Vis spectra of MNPs, M-MIP and M-NIP were obtained in a scan range from 800 to $200 \mathrm{~nm}$ at $600 \mathrm{~nm} / \mathrm{min}$ (Fig. 2.6). $380 \mathrm{~nm}$ was the optimal scan wavelength for all the particles. Satisfactory linearity was achieved for all the particles in a concentration range of 0.005 to $0.1 \mathrm{mg} / \mathrm{mL}$ (Fig. 2.7). The results suggested a feasible UV-Vis analysis for quantitative measurement of M-MIP and M-NIP in water. Magnetic transferability was 


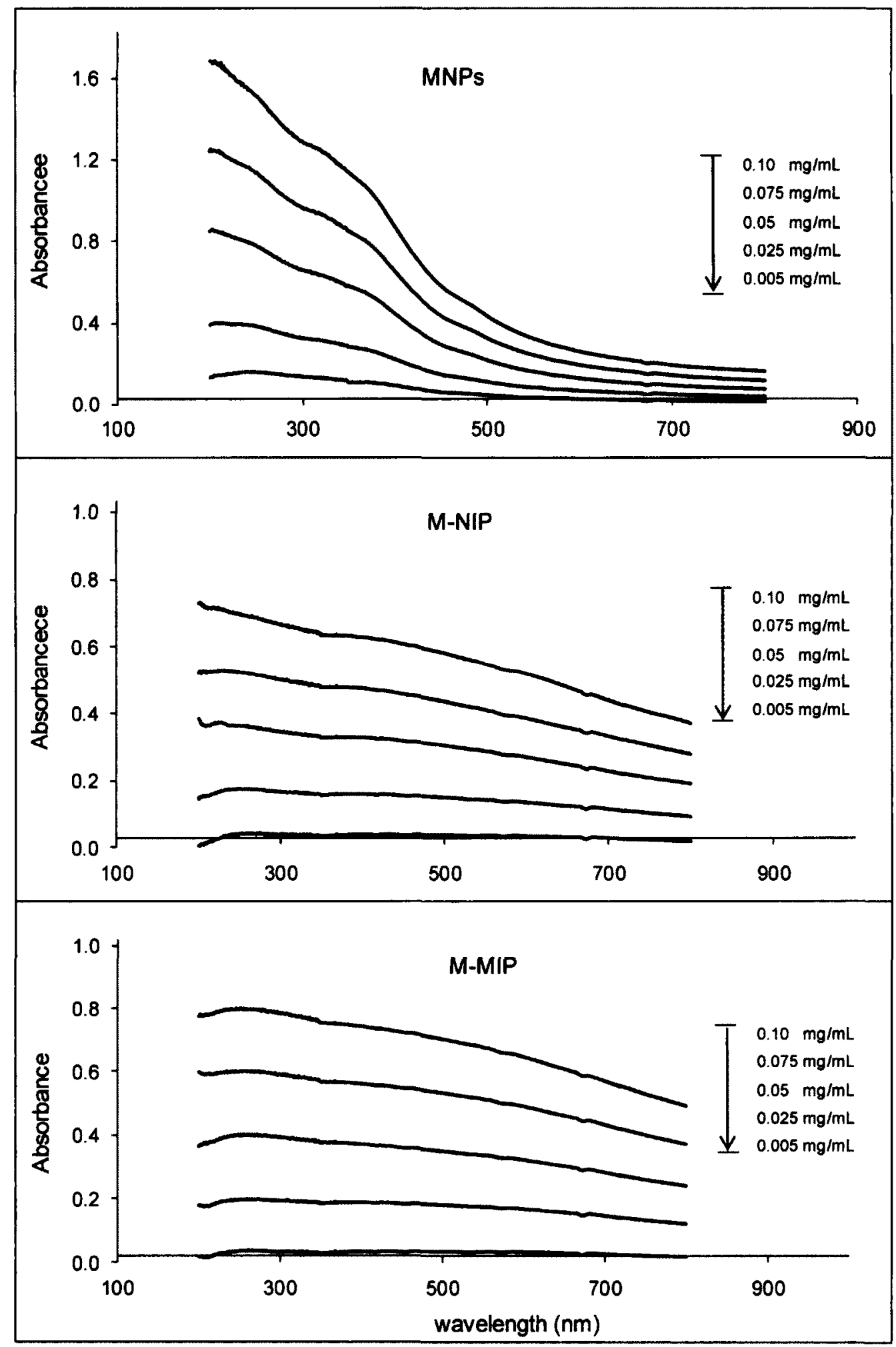

Fig. 2.6 UV-Vis spectra of MNPs, M-NIP and M-MIP 
examined by comparison of concentration before and after magnetic separation with the established calibration curves prepared from known dispersed amounts.

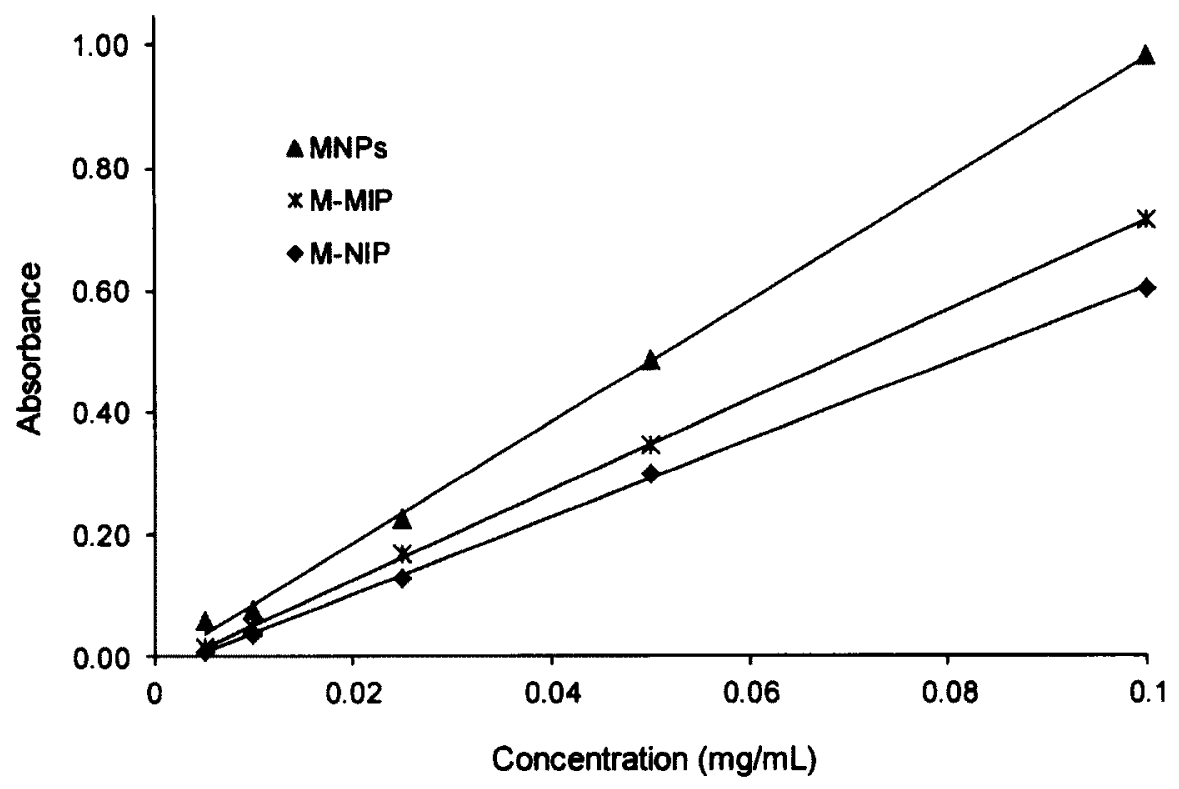

Fig. 2.7 Calibration curves of MNPs, M-MIP and M-NIP

Fig. 2.8 presents \% recovery of the particles versus duration of applied magnetic field. Dynamically, magnetic based separation could be phased into two stages, a quick stage and a slow stage characterized as quickness of magnetic response to the magnetic field. In the first stage, the recovery of magnetic particles was fast. The duration of reaching $>80 \%$ recovery was 5 min, representing a quick magnetically responsive equilibrium between the particles and the applied magnetic source. In the second stage ranging from 5 to 30 min, magnetically response equilibrium was slowly reached. A $\mathbf{3 0}$ min of magnetic separation was able to completely recover M-MIP and MNIP particles from water. Apparently, after surface modification and encapsulation, MMIP and M-NIP remained strong enough magnetic response to allow for effective and rapid magnetic separation to replace the centrifugation process. 


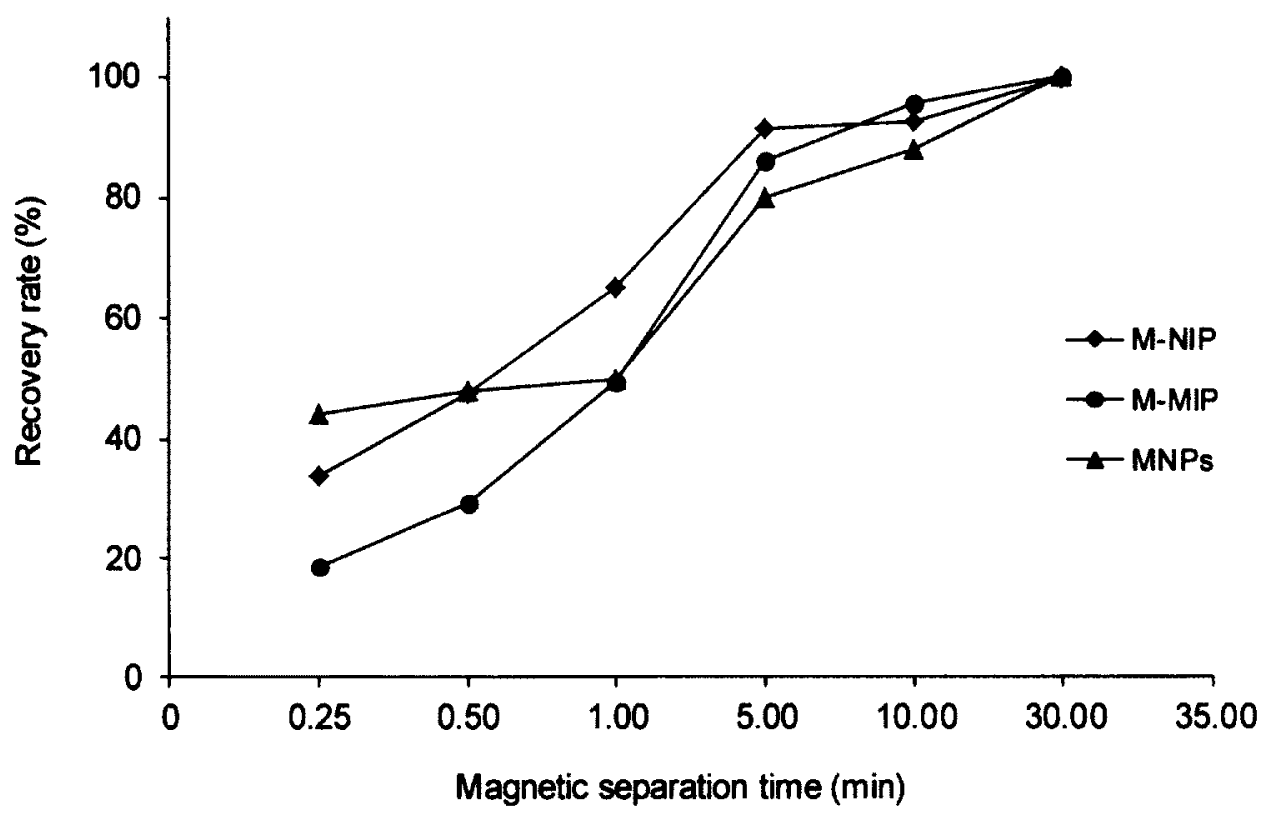

Fig. 2.8 Magnetic recovery of MNPs, M-MIP and M-NIP (initial concentration of M-MIP, M-NIP and MNPs: $0.1 \mathrm{mg} / \mathrm{mL}$ )

\subsubsection{Adsorption performance}

\subsubsection{Quantitative measurement}

An analytical method was developed using LC-MS/MS system to obtain a sensitive and selective analysis of the considered analytes. Quantitative MS/MS analysis was applied in multiple reaction monitoring (MRM) to maximize sensitivity. Water/acetonitrile mobile phase was chosen after comparing chromatographic resolution performance between water/methanol and water/acetonitrile. Ammonia was used as additive to promote deprotonation of free estrogenic molecules [38]. For E2 and BPA, the precursor ion used for MRM was the corresponding deprotonated molecular ion. The most intense product ion was used for quantification. The obtained calibration curves exhibited good linearity in a wide range of concentrations, from 1 to $1000 \mathrm{ng} / \mathrm{mL}$. The E2 residual in the 
supernatant was measured at the end of rebinding. Binding efficiency was calculated as the ratio of (initial concentration - final concentration) / initial concentration, using the LC-MS/MS data acquired with optimized LC-MS/MS conditions.

\subsubsection{Template leakage}

The potential risk of template leakage from M-MIP was assessed before proceeding to rebinding process for aqueous samples. High purity of water obtained from a Milli-Q system was used for template leakage experiment. The test was carried out at $0.5 \mathrm{mg} / \mathrm{mL}$ of M-MIP using the procedure described in experimental section. E2 was not detected in water. Because M-MIP was designed for the removal of estrogenic compounds from aqueous environment, the application of M-MIP as sorbent must meet the background check and there will be no further release of template into water.

\subsubsection{Adsorption analysis}

The recognition ability of E2 imprinted M-MIP toward the target compound was investigated and compared to BPA. The binding efficiency between M-MIP and M-NIP for E2, and M-MIP for E2 and BPA are presented in Fig. 2.9 and Fig. 2.10, respectively.

Fig. 2.9 shows that $0.5 \mu \mathrm{g} / \mathrm{mL}$ of $\mathrm{E} 2$ can be completely removed by $0.5 \mathrm{mg} / \mathrm{mL}$ of M-MIP, showing $100 \%$ binding efficiency at the studied concentration. The result suggested that $1.0 \mathrm{mg}$ of M-MIP was sufficient to quantitatively rebind $1.0 \mu \mathrm{g}$ of E2 from water. M-NIP was able to remove $\sim 0.9 \mu \mathrm{g}$ of E2. M-MIP demonstrated $\sim 10 \%$ higher binding capacity toward E2 than M-NIP. The magnetic hybrids demonstrated improved binding efficiency compared to the MIP sub-micron particles prepared by precipitation polymerization using same polymer materials, in which $5.0-10.0 \mathrm{mg}$ of polymer was in need to completely remove $1.0 \mu \mathrm{g}$ of E2 [22]. The M-MIP possessed binding capacity comparable to the MIP prepared by UV light induced irradiation with MAA - 
trimethylolpropane trimethacrylate composite, a difference of approximate $10 \%$ in adsorption capacity between MIP and NIP particles was obtained at E2 concentration of $2.9 \mu \mathrm{M}$ [39]. Similar binding performance of MIP over NIP prepared with 4-vinylpyridine (4VP) - EGDMA was observed both in aqueous phase and in organic solvent [40].

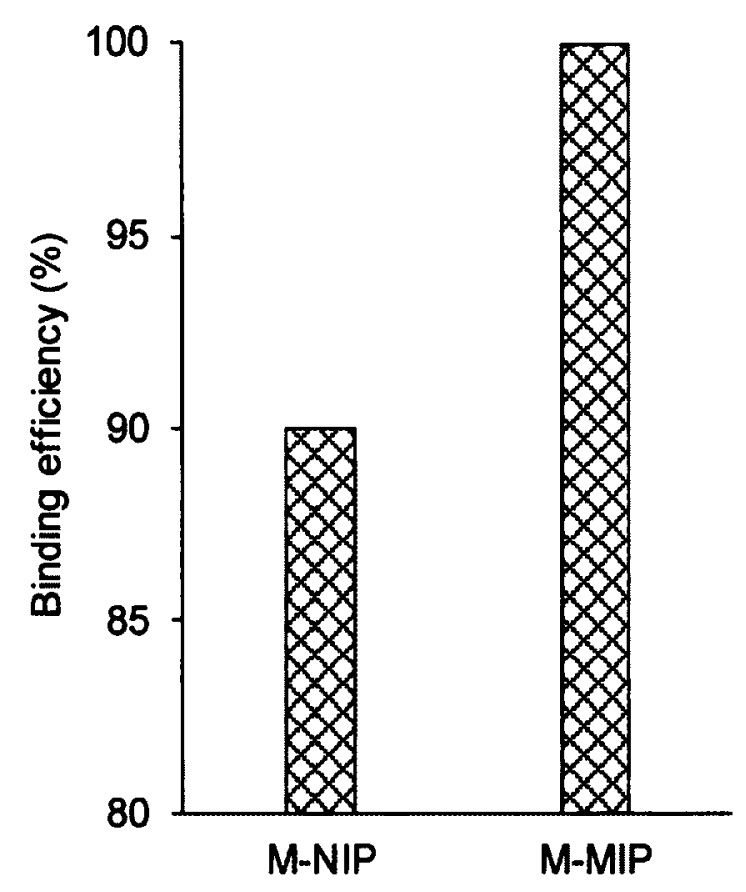

Fig. 2.9 Comparison of binding efficiency of M-MIP and M-NIP for E2 (final concentrations: $0.5 \mu \mathrm{g} / \mathrm{mL}$ of E2; $0.5 \mathrm{mg} / \mathrm{mL}$ of M-MIP, $1 \mathrm{~h}$ incubation)

In this study, the difference in binding capacity between M-MIP and M-NIP was assessed by dispersing same amount $(1 \mathrm{mg})$ of M-NIP or M-MIP to the experimental solution containing same amount of E2 molecules. M-NIP particles have an average size of $200 \mathrm{~nm}$ that is $100 \mathrm{~nm}$ smaller in size and larger in volume to size ratio than M-MIP. The greater specific surface area offers more non-specific binding sites for M-NIP. $10 \%$ difference in binding capacity represents multiple factors determined hydrophobic 
interactions between the adsorbent and adsorbate, and may not reflect the actual binding interactions resulting from specific affinity. It can be expected that in complicated matrices such as natural aqueous environment, the predetermined specific interactions will become dominant.

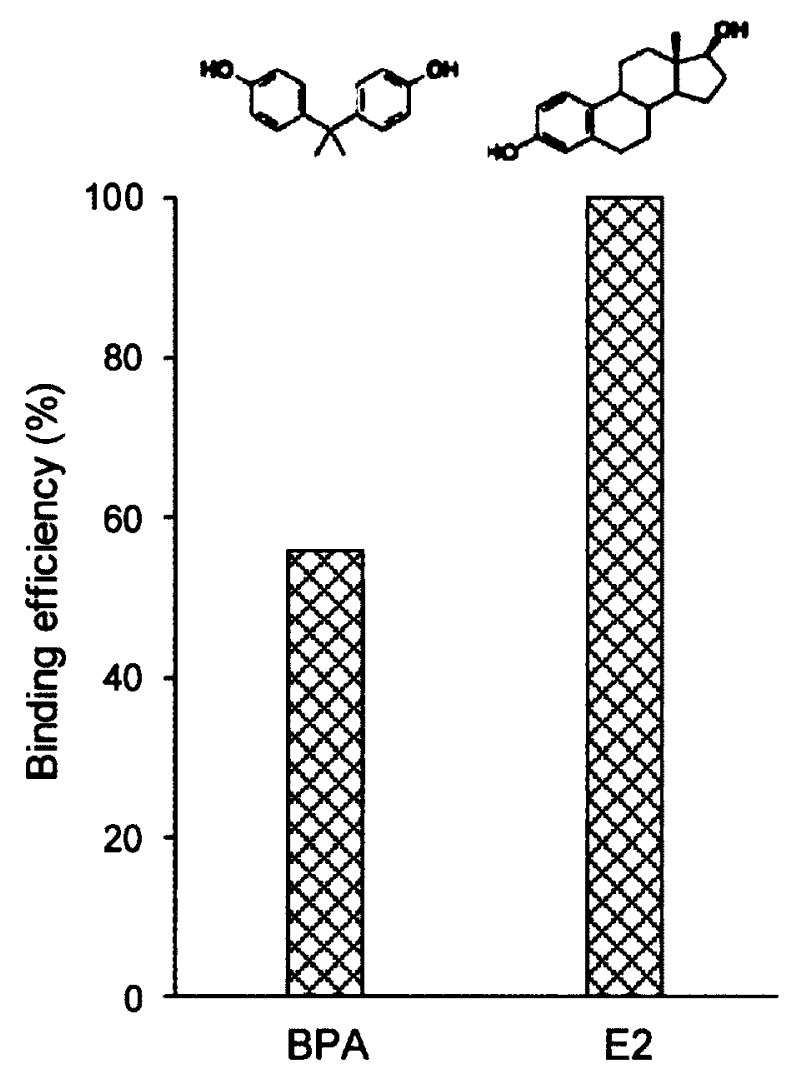

Fig. 2.10 Comparison of binding efficiency of M-MIP for E2 and BPA (final concentrations: $0.5 \mu \mathrm{g} / \mathrm{mL}$ of E2; $0.5 \mathrm{mg} / \mathrm{mL}$ of M-MIP, $1 \mathrm{~h}$ incubation)

Taking into account the size effect, the difference in binding performance between E2 and BPA toward E2 tailor-made M-MIP provides significant binding characteristics with respect to selectivity and specificity. As shown in Fig. 2.10, M-MIP demonstrated as high as $44 \%$ binding efficiency toward E2 than BPA. E2 and BPA both contain two hydroxyl groups (-OH) capable of multiple hydrogen bonding. However, they 
are distinct from each other in size, mass and molecular configuration. Despite the smaller molecular mass, BPA exhibited poor binding interactions with E2 imprinted $M$ MIP. When BPA was used as interfering or competing compound, similar pattern of binding was observed $[14,37,41]$. The binding performance further suggests the potential application of E2 imprinted M-MIP for the removal of structurally related steroid estrogens from aqueous environment.

\subsubsection{Application}

To assess the applicability and practicality, tap water samples were spiked at 0.5 $\mu \mathrm{g} / \mathrm{mL}$ of E2. M-MIP was used as sorbent at a concentration of $0.5 \mathrm{mg} / \mathrm{mL}$ for recovery test using the established procedure. Tap water blanks were also analyzed for background check. The calculated \% recovery is summarized in Table 2.1. A satisfactory recovery of $>95 \%$ was achieved. The obtained results indicated that E2 imprinted $M$ MIP was able to produce good recovery and could be applied potentially for the removal of E2 from aqueous environment.

Table 2.1 Recovery of spiked E2 from tap water

\begin{tabular}{cccc}
\hline $\begin{array}{c}\text { Spiked } \\
\text { sample }\end{array}$ & $\begin{array}{c}\text { Spiked concentration } \\
(\mathrm{ng} / \mathrm{mL})\end{array}$ & $\begin{array}{c}\text { Detected concentration } \\
\text { after binding }(\mathrm{ng} / \mathrm{mL})\end{array}$ & $\begin{array}{c}\text { Calculated recovery } \\
(\%)\end{array}$ \\
\hline Sample 1 & 500.0 & 20.8 & 95.9 \\
Sample 2 & 500.0 & 21.3 & 95.8 \\
\hline
\end{tabular}




\subsection{Conclusions}

Estrogen imprinted MIP was encapsulated on surfactant-modified magnetic nanoparticles for the generation of core-shell MIP by ultrasonication assisted synthesis. The resultant particles, featuring fine sizes and narrow size distribution, possessed strong magnetic transferability. The recognition ability and adsorption capacity of the imprinted layer toward the target molecule were satisfactory. The incorporation of magnetic properties with imprinted effect by ultrasonication process presented a novel approach for synthesis of magnetic MIP without significantly compromising the ability of magnetic response of MNPs and the selectivity of MIP. The research revealed that it is essential to modify magnetic particles in a compatible medium to facilitate polymer growth around the magnetic core and promote an even imprinted sites distribution on shell.

The binding performance suggested the applicability and practicality of the magnetic hybrids in aqueous environments at trace concentrations. The proposed protocol demonstrated the feasibility for rapid and selective removal of EDCs in an environmentally friendly separation, which has the potential for water treatment to address the increasing public concerns arising from these emerging pollutants. The research work may be further extended by incorporating imprinted surfaces for designing MIPs for various indented applications. 


\section{References}

1. J. Ashby, Environ. Toxicol. Phar., 3, 87 (1997).

2. M. Clara, N. Kreuzinger, B. Strenn, O. Gans, H. Kroiss, Water Res., 39, 97 (2005).

3. J.P. Sumpter, A.C. Johnson, Environ. Sci. Technol., 39, 4321 (2005).

4. L. Chen, S. Xu, J. Li, Chem. Soc. Rev., 40, 2922 (2011).

5. C. Alexander, H.S. Andersson, L.I. Andersson, R.J. Ansell, N. Kirsch, I.A. Nicholls, J. O'Mahony, M.J. Whitcombe, J. Mol. Recognit., 19, 106 (2006).

6. K. Mosbach, O. Ramström, Nat. Biotechnol., 14, 163 (1996).

7. M. Faraji, Y. Yamini, M. Rezaee, J. Iran. Chem. Soc., 7, 1 (2010).

8. A.E. Rachkov, S.H. Cheong, A.V. El'skaya, K. Yano, I. Karube, Polym. Advan. Technol., 9, 511 (1998).

9. L. Ye, P.A.G. Cormack, K. Mosbach, Anal. Commun., 36, 35 (1999).

10. L. Ye, R. Weiss, K. Mosbach, Macromolecules, 33, 8239 (2000).

11. S. Wei, A. Molinelli, B. Mizaikoff, Biosens. Bioelectron., 21, 1943 (2006).

12. Z. Meng, W. Chen, A. Mulchandani, Environ. Sci. Technol., 39, 8958(2005).

13. Y. Watabe, T. Kubo, T. Nishikawa, T. Fujita, K. Kaya, K. Hosoya, J. Chromatogr. A, 1120,252 (2006).

14. J. Ma, L. Yuan, M. Ding, S. Wang, F. Ren, J. Zhang, S. Du, F. Li, X. Zhou, Biosens. Bioelectron., 26, 2791 (2011).

15. Y. Li, C. Dong, J. Chu, J. Qi, X. Li, Nanoscale, 3, 280 (2011).

16. N. Saifuddin, Y.A.A. Nur, S.F. Abdullah, Asian J. Biochem., 6, 38 (2011).

17. M. Le Noir, F.M. Plieva, B. Mattiasson, J. Separ. Sci., 32, 1471 (2009).

18. O. Nemulenz, B. Mhaka, E. Cukrowska, O. Ramström, H. Tutu, L. Chimuka, J. Separ. Sci., 32, 1941 (2009).

19. J.C. Bravo, P. Fernandez, J.S. Durand, Analyst, 130, 1404 (2005). 
20. M. Le Noir, A.S. Lepeuple, B. Guieysse, B. Mattiasson, Water Res., 41, 2825 (2007).

21. Z. Zhang, J. Hu, Water Air Soil Poll., 210, 255 (2010).

22. E.P.C. Lai, Z. De Maleki, S. Wu, J. Appl. Polym. Sci., 116, 1499 (2010).

23. Y. Lin, Y. Shi, M. Jiang, Y. Jin, Y. Peng, B. Lu, K. Dai, Environ. Pollut., 153, 483 (2008).

24. X. Wang, L. Wang, X. He, Y. Zhang, L. Chen, Talanta, 78, 327 (2009).

25. Y. Lin, X. Li, J. Chu, C. Dong, J. Qi, Y.Yuan, Environ. Pollut., 158, 2317 (2010).

26. P. Berger, N.B. Adelman, K.J. Beckman, D.J. Campbell, A.B. Ellis, G.C. Lisensky, J. Chem. Educ., 76, 943 (1999).

27. N.T. Ha, N.H. Hai, N.H. Luong, N. Chau, H.D. Chinh, VNU J. Sci. Nat. Sci. Technol., 24, 9 (2008).

28. I.C. Schoonover, G.M. Brauer, and W.T. Sweeney, J. Res. Nat. Bur. Stand., 49, 359 (1952).

29. C.K. Ober, M.L. Hair, J. Polym. Sci. Pol. Chem., 25, 1395 (1987).

30. J. Svenson, Anal. Lett., 39, 2749 (2006).

31. R. Hoogenboom, U.S. Schubert, Macromol. Rapid Comm., 28, 368 (2007).

32. Y. Hu, Y. Li, R. Liu, W. Tan, G. Li, Talanta, 84, 462 (2011).

33. X. Zheng, G.M. Murray, Separ. Sci. Technol., 31, 2403 (1996).

34. M.D. Luque de Castro, F. Priego-Capote, Talanta, 72, 321 (2007).

35. J. Chatterjee, Y. Haik, C. Chen, J. Magn. Magn. Mater., 257, 113 (2003).

36. L. Suber, P. Imperatori, G. Ausanio, F. Fabbri, H. Hofmeister, J. Phys. Chem. B, $109,7103(2005)$

37. A. Hrdina, E. Lai, C. Li, B. Sadi, G. Kramer, J. Magn. Magn. Mater., 322, 2622 (2010).

38. A. Gentili, D. Perret, S. Marchese, R. Mastropasqua, R. Curini, A.D. Corcia, 
Chromatographia, 56, 25 (2002).

39. Z. Zhang, J. Hu, Water Res., 42, 4101(2008).

40. M. Le Noir, B. Guieysse and B. Mattiasson, Water Sci. Technol., 53, 205 (2006).

41. Z. DeMaleki, E.P.C. Lai and E. Dabek-Zlotorzynska, J. Separ. Sci., 33, 2796 (2010). 


\section{Chapter 3}

Dual-Template Imprinted Magnetic Polymer Nanoparticles for Class-Specific Removal of Endocrine Disrupting Compounds from Aqueous Environment

\subsection{Introduction}

The newly recognized, unregulated emerging pollutants in the environment have received increasing attention of the public, scientific community and regulation bodies [1]. Among the list of emerging pollutants are endocrine disrupting compounds (EDCs), characterized as chemical disruptors that can mimic or block the actions of natural hormones in living organisms and impair their normal functioning [2-4]. The vast amount of data on adverse effects of EDCs has been reported for various fish species; studies have linked EDCs with numerous health risks and concluded a significant adverse toxicological effect on living organisms' endocrine system at very low concentration level [5-10].

Steroid estrogens and phenolic disruptors are of particular concern among EDCs due to the consumption volume as well as their estrogenic activity. The main substances causing estrogenic effect are the natural estrogens - estrone (E1), 17ß-estradiol (E2), estriol (E3) and synthetic estrogen - ethinylestradiol (EE2). Phenolic disruptors, including bisphenol A (BPA), diethylstilbestrol (DES) and 4-n-nonylphenol etc., have a less estrogenic activity but their environmental existence are generally found at a higher level than steroid estrogens [11]. These compounds are considered representative EDCs in most environmental studies.

EDCs are discharged into the environment as a result of rapidly increasing production and wide use of manufactured materials [12]. Industrial effluents, residential discharges, municipal wastes and agricultural run-offs are responsible for the presence 
of EDCs in aqueous environment [13]. Human excrements, animal wastes and synthetic substances such as pesticides, personal care products and hormonal pharmaceuticals contribute to water pollution. Exposure to EDCs is predominantly oral. Therefore, there is increasing concern regarding the intake of EDCs through environmental waters. The development of innovative and effective techniques for the reduction of estrogenic effect from aqueous environment has become priority under the pressure of public concern and demand.

Molecularly imprinted polymers (MIPs) are synthetic sorbents that can act as artificial receptors. MIPs have been developed with specific templates for the selective extraction of various molecules in complex matrices such as pharmaceuticals in clinical analysis, contaminants in food samples, and organic micro-pollutants in environmental analysis $[14,15]$. MIPs are generally prepared by polymerizing a template-monomer complex with a cross-linker. After template removal, binding sites complementary in structure and chemical functionality to the template molecules are generated. These sites have a degree of flexibility in their recognition properties and can be selective toward other compounds with similar structures or physicochemical properties [16], leading to the formation of MIPs that possess class-specificity toward a group of molecules.

The removal of EDCs from aqueous environment is one of the actively studied areas. BPA imprinted polymer was investigated for the selective removal of phenolic EDCs such as bisphenol $C$, bisphenol $Z$ and DES [17]. BPA-4VP-ethylene glycol dimethacrylate (EGDMA) MIP for removing several phenolic compounds and phenoxyacid herbicides was studied in the presence of other pesticides. Several parameters affecting extraction efficiency were evaluated to achieve a selective preconcentration of these compounds from aqueous samples [18]. E2 imprinted MIP was shown to be effective in removing other structurally related estrogens [19]. On-column 
solid-phase extraction using DES imprinted MIP was assessed for its selectivity toward several estrogens including E1, E2, E3, and EE2 [20]. MIP microspheres imprinted with a-estradiol exhibited outstanding affinity toward the template and showed significant binding affinity toward other compounds (E2, E3, EE2 and DES) [21].

These studies demonstrated the potential in the extraction of different groups of EDCs. However, EDCs removal with single template MIPs has its limitations. In most cases, steroid estrogen imprinted MIPs exhibit poor adsorption toward phenolic disruptors, or vice versa. In this research, the removal of steroid and phenolic EDCs from aqueous environment was proposed by using a dual-template imprinted MIP. The binding characteristic was evaluated using two groups of EDCs. The effect of environmental variables on adsorption efficiency and the reusability were studied in detail. The effectiveness of magnetic separation was studied in a quantitative manner. The suitability and applicability of class-specific removal of EDCs were verified using river water samples spiked with steroid and phenolic EDCs at trace concentration levels.

\subsection{Experimental}

\subsubsection{Materials}

E1, E2, E3, BPA, DES, 4VP, EGDMA, methacrylic acid (MAA) and methacrylamide (MAM) were purchased from Aldrich (St. Louis, MO, USA). 2, 2'-azobis (2methylpropionitrile) (AIBN) was supplied by Pfaltz \& Bauer (Waterbury, CT, USA). Phenol (PH) was obtained from BHD Chemicals Ltd. (Poole, England). Hydroquinone (HQ) was from Sigma (St. Louis, MO, USA). Oleic acid (OA) and diclofenac sodium were purchased from Sigma-Aldrich (Oakville, ON, Canada). Sodium dodecyl sulfate (SDS) was from Pierce (Rockford, IL, USA). Humic acid (HA) was obtained from Alderich (Milwaukee, WI, USA). All chemicals and reagents were used as purchased. Diclofenac 
sodium was acidified with $\mathrm{HCl}$ and extracted in chloroform (DFC) [22]. High quality water was obtained from a Millipore Q-POD system (MA, USA). Individual EDCs stock solutions were prepared at $1000 \mu \mathrm{g} / \mathrm{ml}$ in methanol and stored at $-20{ }^{\circ} \mathrm{C}$, from which working standards were prepared at different concentrations.

\subsubsection{Synthesis of dual-template imprinted M-MIP}

\subsubsection{Preparation of aqueous ferrofluid}

Aqueous ferrofluid was prepared through surface modification of $\mathrm{Fe}_{3} \mathrm{O}_{4}$ magnetic nanoparticles (MNPs) with OA and SDS surfactants. MNPs were dispersed in deionized distilled water $(\mathrm{DDW})$ at $60^{\circ} \mathrm{C}$, followed by the addition of $\mathrm{OA}$ with ultrasonication assistance. SDS aqueous solution was then introduced at ambient temperature. The final concentration of $\mathrm{Fe}_{3} \mathrm{O}_{4}$ was $5 \mathrm{mg} / \mathrm{mL}$.

\subsubsection{Preparation of pre-polymerization solution}

Pre-polymerization complex was prepared in acetone/acetonitrile $(1: 3, v / v) .34 .1$ $\mathrm{mg}$ of E2 was added into a glass vessel containing $5 \mathrm{~mL}$ of solvent, $43 \mu \mathrm{L}$ of MAA was then introduced and well mixed. Similarly, a $5-\mathrm{ml}$ solution containing $28.6 \mathrm{mg}$ of BPA and $43 \mu \mathrm{L}$ of MAA was prepared. The solutions were kept in the dark for $2 \mathrm{~h}$. Prepolymerization solution was prepared by combining the two complexes, followed by the addition of $317 \mu \mathrm{L}$ of EGDMA.

\subsubsection{Ultrasonication assisted synthesis}

A 20-ml polymerization solution with equal volumes of aqueous ferrofluid and prepolymerization solution was prepared with $1 \mathrm{~min}$ of sonication degassing. $11.5 \mathrm{mg}$ of initiator, AIBN, was added, followed by a 7-min nitrogen purging. The glass vessel was capped and firmly sealed with parafilm before a 2-h polymerization in an ultrasonication 
bath (Bransonic ultrasonicator, 2510R-DTH, USA) at $65^{\circ} \mathrm{C}$. M-MIP was collected by magnetic field, followed by several rounds of template removal in acetic acid/methanol $(1: 9, v / v)$. M-NIP was prepared in the same way without the templates.

\subsubsection{Characterization}

\subsubsection{SEM scans}

The morphology and structure of E2-BPA M-MIP particles were examined by scanning electron microscopy (SEM) (Tescan-Vega II-XMU, Czech). Dried sample was coated with gold/palladium to create a conductive surface using an Anatech Hummer VII Sputter Coater (VA, USA).

\subsubsection{FT-IR scans}

Fourier transform infrared spectroscopy (FT-IR) analysis was carried out on a FTIR spectrometer (Varian $1000 \mathrm{FT}-\mathrm{IR}$, USA). Dried M-MIP and $\mathrm{KBr}$ mixture was pressed to form pellets for FT-IR scan. The spectrum of MAA and EGDMA was acquired using liquid samples sandwiched between $\mathrm{KBr}$ pellets.

\subsubsection{Magnetic properties}

Magnetic properties were examined on an Abraxis 60-tube magnetic separator (PA, USA) and an UV-Vis spectrophotometer (Varian Cary 3, USA). Stock solutions were prepared with DDW at a concentration of $0.1 \mathrm{mg} / \mathrm{mL}$, from which a series of working solutions were prepared. The amount of remained particles in solution was measured by UV-Vis absorbance.

\subsubsection{Kinetic properties}

Kinetic properties were examined on an UV-Vis spectrophotometer and a turbidity 
meter (Aquafast AQ3010, USA). The turbidity meter was calibrated using the standards at $0.02,20.0,100.0$ and 800.0 Nephelometric Turbidity Units (NTU). DDW was used for sample preparation and as control blank. $500 \mathrm{~mL}$ of aqueous suspensions containing 5.0 $\mathrm{mg} / \mathrm{L}$ of M-MIP was freshly prepared with 15-min sonication and analyzed within $24 \mathrm{~h}$.

\subsubsection{Adsorption performance}

\subsubsection{Analytical method}

High performance liquid chromatography-tandem mass spectrometry (LC-MS/MS) analysis was conducted on an AB Sciex API 2000 triple quadrupole tandem mass spectrometer coupled with a Shimadzu Prominence liquid chromatograph using an analytical column (Shimadzu, $50 \times 4.6 \mathrm{~mm}, 5.0 \mu \mathrm{m}, \mathrm{MD}, \mathrm{USA}$ ) at ambient temperature. The main MS/MS parameters were optimized by direct infusion of standards dissolved in water/acetonitrile. The MS/MS was performed using Electrospray lonization (ESI) probe in negative polarity. Mass spectra were acquired in multiple reaction monitoring (MRM) mode. LC flow rate was set at $0.3 \mathrm{~mL} / \mathrm{min}$ with a water/acetonitrile mobile phase running at a non-linear gradient elution of a $25-\mathrm{min}$ cycle. The residuals in the solutions were measured at the end of incubation after magnetic separation.

\subsubsection{Effect of environmental variables on adsorption efficiency}

The effect of environmental variables, including ionic strength, $\mathrm{HA}, \mathrm{pH}$ and temperature, on the adsorption efficiency was investigated at environmentally related levels. 2-mL incubation solutions were prepared at a final concentration of $0.05 \mathrm{mg} / \mathrm{mL}$ of M-MIP and $0.5 \mu \mathrm{g} / \mathrm{mL}$ of EDCs.

$0.1 \mathrm{M} \mathrm{NaCl}$ was introduced to prepare solutions with various $\mathrm{NaCl}$ concentrations at 2.0,5.0,10.0,25.0 mM. Aqueous humic acid was used to create a series of artificial environmental samples with final HA concentrations at 2.0,5.0, 8.0, $10.0 \mathrm{mg} / \mathrm{L}$. $\mathrm{pH}$ was 
adjusted between $3.5-11.0$ through the addition of $0.1 \mathrm{M} \mathrm{NaOH}$ or $\mathrm{HCl}$. Temperature effect was studied by placing incubation solutions at ambient environment and in water bath. Temperatures were controlled at $20 \pm 0.5^{\circ} \mathrm{C}$ and $40 \pm 0.5^{\circ} \mathrm{C}$.

\subsubsection{Equilibrium adsorption}

Equilibrium adsorption was assessed by suspending $1.0 \mathrm{mg}$ of M-MIP into aqueous solutions containing various concentrations of EDCs for incubation. The final concentrations of EDCs were at $0.25,0.50,0.75,1.00,1.25,1.75,2.00,4.00 \mu \mathrm{g} / \mathrm{mL}$. Samples were sonicated for $5 \mathrm{~min}$ and shaken for $6 \mathrm{~h}$ at $120 \mathrm{rpm}$. M-NIP was treated similarly to compare equilibrium adsorption.

\subsubsection{Adsorption tests on spiked environmental samples}

$2 \mathrm{~mL}$ of tap water and river water samples were applied to $1.0 \mathrm{mg}$ of M-MIP to evaluate sample matrix effect. Adsorption tests were carried out by spiking EDCs at a final concentration of $1.0 \mu \mathrm{g} / \mathrm{mL}$ for each compound. Samples were sonicated for $5 \mathrm{~min}$, followed by $1 \mathrm{~h}$ incubation. A 30-min magnetic separation was conducted on the Abraxis separator at the end of incubation. The residual EDCs were analyzed with LC-MS/MS. In the final phase, $100 \mathrm{~mL}$ of river water samples was spiked with trace EDCs $(10 \mu \mathrm{g} / \mathrm{L}$, final) and incubated with $50 \mathrm{mg}$ of M-MIP.

\subsubsection{Reuse and regeneration}

$2.0 \mathrm{mg}$ of M-MIP was initially incubated with E2 and BPA $(1.0 \mu \mathrm{g} / \mathrm{mL}$, final) for $1 \mathrm{~h}$ in a 5-ml aqueous solution. After each use, M-MIP was regenerated with subsequential acetic acid/methanol $(4 \times 5 \mathrm{ml}, 1: 9, \mathrm{v} / \mathrm{v})$ and methanol $(3 \times 5 \mathrm{ml})$ washing with $20-\mathrm{min}$ sonication. After drying in vacuum, the regenerated M-MIP was reused. Magnetic separation was maintained at constant 30 min for all processes. 


\subsection{Results and discussion}

\subsubsection{Synthesis of dual-template M-MIP}

MIPs can be synthesized to exhibit class specificity [23]. In addition, MIPs containing multiple binding sites can be prepared using two or more templates [24-26]. In this work, a dual-template imprinted MIP layer was formed on a magnetic core using the non-covalent imprinting approach [27].

MIP was synthesized by polymerizing E2-MAA and BPA-MAA template-monomer complexes with a common cross linker (EGDMA). MAA is a widely used functional monomer for its unique characteristics - capable of acting as a hydrogen-bond donor and acceptor, and suitable for ionic interactions [28]. Steroid estrogens (E1, E2 and E3) and phenolic disruptors (BPA and DES) consist of two or more hydroxyl/carbonyl groups in their molecules (Fig. 3.1). Hydrogen bonding results in the formation of a complex between the estrogenic molecule and functional monomer molecules.

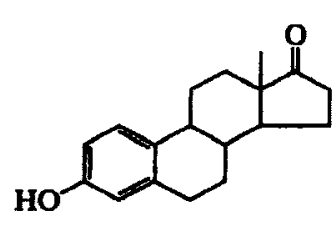

E1<smiles>CC12CCC3c4ccc(O)cc4CCC3C1CCC2O</smiles>

E2<smiles>CC12CCC3c4ccc(O)cc4CCC3C1CC(O)C2O</smiles>

E3<smiles>CC(C)(c1ccc(O)cc1)c1ccc(O)cc1</smiles>

BPA<smiles>CCC(=C(CC)c1ccc(O)cc1)c1ccc(O)cc1</smiles>

DES<smiles>C=C(C)C(=O)O</smiles>

MAA<smiles>C=C(C)C(N)=O</smiles>

MAM<smiles>C=Cc1ccncc1</smiles>

4VP

Fig. 3.1 Structures of the studied EDCs and monomers 
In our previous work, an E2-MAA-EGDMA MIP layer was successfully coated on a magnetic core using an ultrasonication assisted synthetic route. The resultant M-MIP particles exhibited spherical morphology, strong magnetic property and satisfactory rebinding efficiency toward E2. However, E2 M-MIP performed poorly in BPA adsorption. To enable efficient binding of steroid and phenolic EDCs, E2 and BPA were chosen as templates to prepare an E2-BPA dual-template MIP that has functionality complementary to both steroid and phenolic EDCs. Two different imprinting approaches were attempted using dual-template/dual-monomer and dual-template/single-monomer complexes with EGDMA for a comparison of the resultant MIPs.

4VP and MAM were tested to complex BPA for preparing MIP that can be integrated into E2-MAA MIP. 4VP has been reported to prepare BPA-4VP MIPs [29, 30]. MAM, a monomer that contains $\mathrm{C}=\mathrm{O}$ functional group, can form a BPA-MAM complex through hydrogen bonding. Polymerization was performed in a water bath at $65^{\circ} \mathrm{C}$ for 18 $h$ in a hydro-organic medium $(1: 1, v / v)$. The preliminary adsorption tests showed that BPA-4VP MIP displayed an $8 \%$ higher binding capacity than BPA-MAM MIP. Hence, the BPA-4VP combination was selected for incorporation into E2-MAA-EGDMA MIP in comparison to (E2-MAA+BPA-MAA)-EGDMA MIP. The binding results showed that (E2MAA+BPA-MAA)-EGDMA MIP and (E2-MAA+BPA-4VP)-EGDMA MIP had same degree of recognition toward E2 and BPA.

In the final tests, ultrasonication assisted synthesis was applied using magnetic ferrofluid and the selected MIP. The yield of M-MIP was significantly low using (E2MAA+BPA-4VP)-EGDMA dual-template/dual-monomer recipe. A separate investigation showed that plenty polymer particles, visualized by the cloudiness of polymerization solution, were observed around $15 \mathrm{~min}$ after E2-MAA-EGDMA polymerization began. While abundant polymer particles were not present until around 45 min after polymerizing BPA-4VP-EGDMA complex, suggesting that the polymerization of 4VP- 
EGDMA and MAA-EGDMA was not synchronized. MAA single monomer for the preparation of E2-BPA imprinted M-MIP was finalized after comparing the yield, binding efficiency and particle uniformity. Fig. 3.2 illustrates the synthetic route of E2-BPA MMIP.

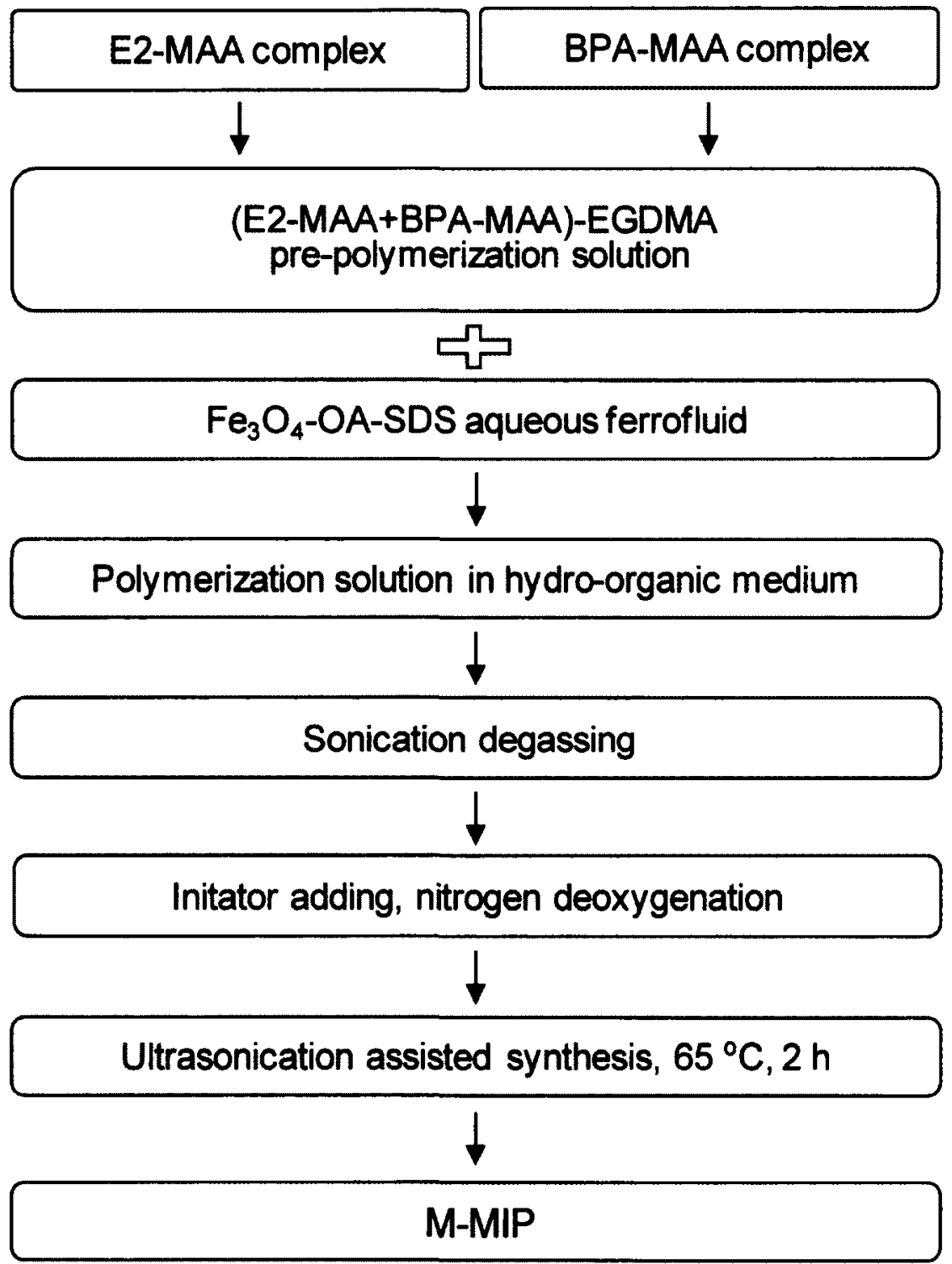

Fig. 3.2 Synthetic route of E2-BPA imprinted M-MIP 


\subsubsection{Characterization}

\subsubsection{Particle size and morphology}

The particles synthesized with ultrasonication assistance exhibit spherical morphology. Satisfactory uniformity with narrow size distribution was observed through SEM images (Fig. 3.3). The $\mathrm{Fe}_{3} \mathrm{O}_{4}-\mathrm{OA}-\mathrm{SDS}, \mathrm{M}-\mathrm{NIP}$ and M-MIP particles have average size at 27,200 and $270 \mathrm{~nm}$, respectively. A polymer layer with an average thickness of $120 \mathrm{~nm}$ is encapsulated on the magnetic core. The difference in particle size by $70 \mathrm{~nm}$ between M-NIP and M-MIP indicates the existence of imprinted cavities on polymer shell.

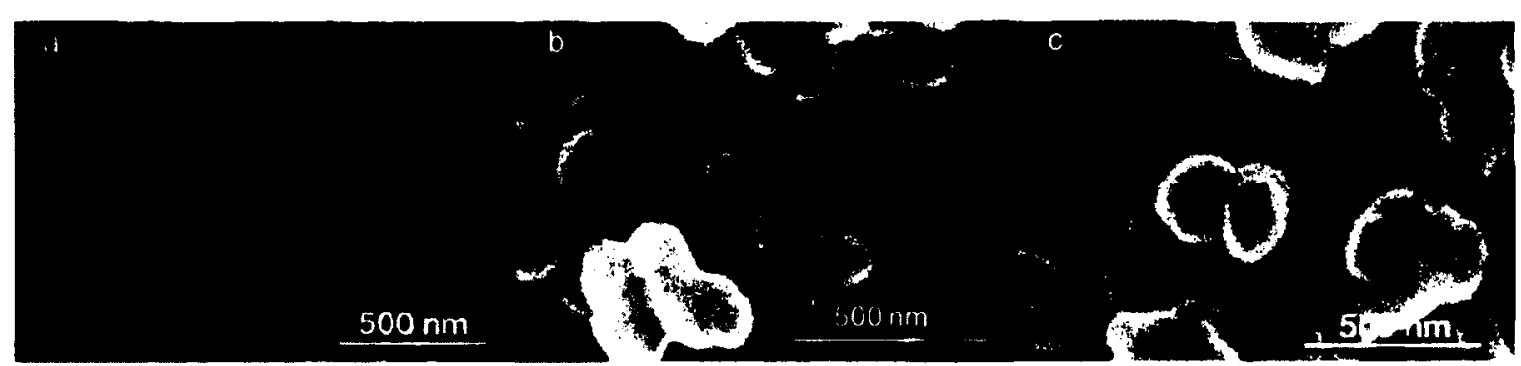

Fig. 3.3 SEM scans of $\mathrm{Fe}_{3} \mathrm{O}_{4}-\mathrm{OA}-\mathrm{SDS}(\mathrm{a})$, M-NIP (b) and M-MIP (c)

\subsubsection{FT-IR spectra}

Fig. 3.4 presents the FT-IR spectrum of MAA, EGDMA and M-MIP obtained through scanning M-MIP and free MAA and EGDMA samples. The individual peaks and the relative intensity reveal the polymer composition and, importantly, the nature of bonds that are formed on the imprinted layer.

The band at $1697 \mathrm{~cm}^{-1}$ indicates $\mathrm{C}=0$ stretch vibrations of MAA; $1723 \mathrm{~cm}^{-1}$ represents $\mathrm{C}=\mathrm{O}$ bond of EGDMA. Attributed to the $\mathrm{O}-\mathrm{H}$ stretch vibration, MAA has a broad peak band at $2958 \mathrm{~cm}^{-1}$. Free MAA and EGDMA have peak at $1637 \mathrm{~cm}^{-1}$, corresponding to the $\mathrm{C}=\mathrm{C}$ bond of MAA and EGDMA [31]. 


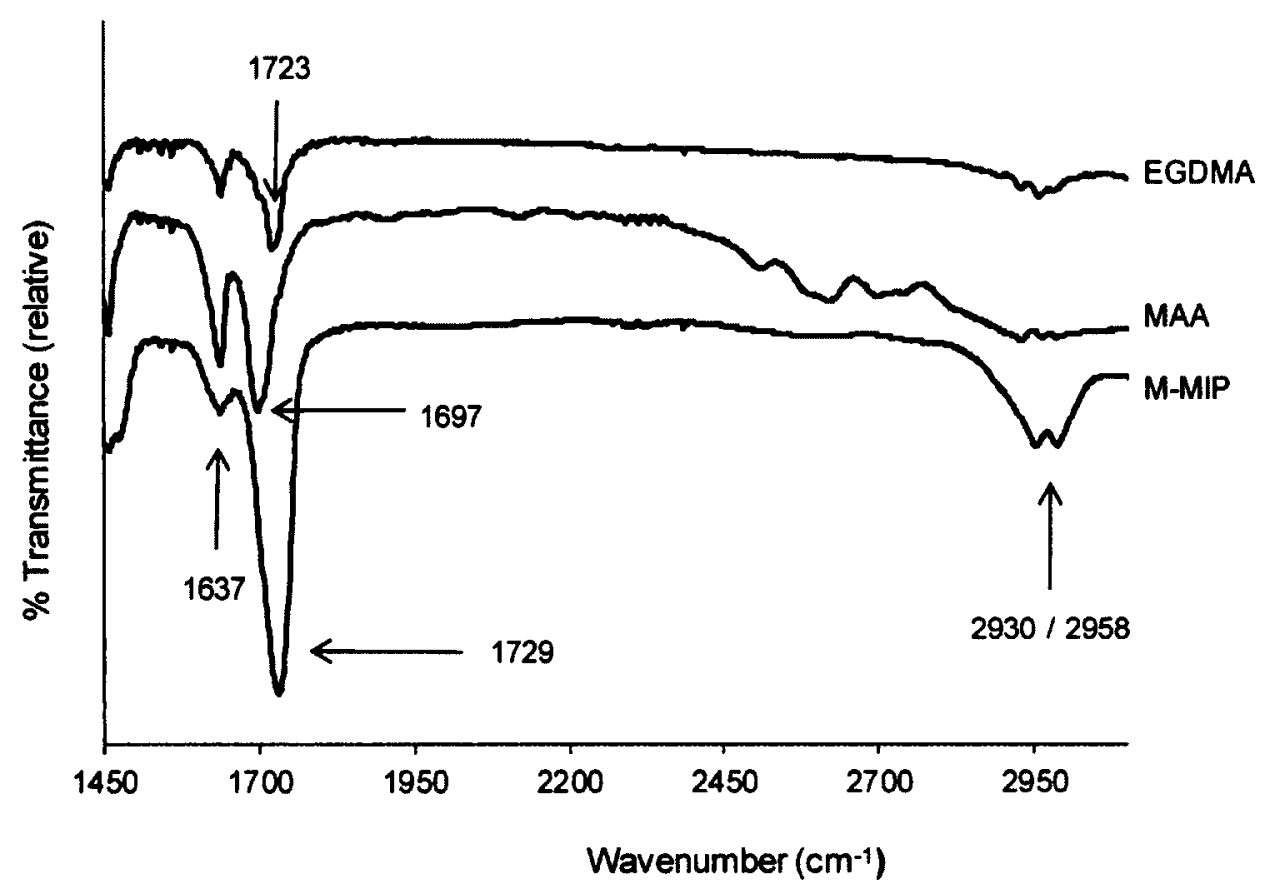

Fig. 3.4 FT-IR spectrum of MAA, EGDMA and M-MIP

It is obvious from Fig. 3.4 that M-MIP is characterized by a very weak peak at 1637 $\mathrm{cm}^{-1}$ and a strong, sharp peak band at $1729 \mathrm{~cm}^{-1}$. Comparing to the strong peak at 1637 $\mathrm{cm}^{-1}$ of MAA, M-MIP has very weak $C=C$ vibrations, suggesting that the $C=C$ double bond is broken up after polymerization. This is in conjunction with the two distinct peaks at 2930 and $2958 \mathrm{~cm}^{-1}$, resulting from C-H stretching vibrations. The high intensity peak near $1700-1730 \mathrm{~cm}^{-1}$ indicates the existence of a large number of carbonyl functional groups $(C=0)$ derived from MAA and EGDMA $[32,33]$. The existence of these characteristic peaks in M-MIP shows the successful encapsulation of polymeric layer on magnetic core. The presence of $-\mathrm{OH}$ and $\mathrm{C}=\mathrm{O}$ groups on the coated layer represents the capability of hydrogen bonding interactions of M-MIP. 


\subsubsection{Kinetic properties of M-MIP particles}

The study on the kinetic properties of M-MIP would provide information with respect to M-MIP particles motion, collision and, importantly, suspension-settlement pattern that are important to the subsequent development of binding and separation procedures. The kinetic properties of M-MIP particles were studied using UV-Vis technique for a 4-h data acquisition and process. After $24 h$, the absorbance on the same sample (initial concentration of M-MIP: $5.0 \mathrm{mg} / \mathrm{L}$ ) was measured. The observed absorbance variations are plotted against time at 2-min intervals for $1 \mathrm{~h}$ (Fig. 3.5a), 30min intervals for $4 \mathrm{~h}$ (Fig. 3.5b) and the overall changes over $24 \mathrm{~h}$ (Fig. 3.5c). The absorbance decreased by $9 \%$ in $1 \mathrm{~h}$. Thereafter, a gradual decrease in absorbance was observed. In $4 \mathrm{~h}$, the decrease in absorbance was $13 \%$. The absorbance decreased by $43 \%$ over $24 \mathrm{~h}$.

Turbidity variations provide settling characteristics of the suspended M-MIP particles from a different view. The initial turbidity for $5.0 \mathrm{mg} / \mathrm{L}$ of M-MIP suspension was $11.60 \mathrm{NTU}$. It decreased slightly to 11.50 in $1 \mathrm{~h}$ (Fig. 3.6a). From 1 to $4 \mathrm{~h}$ (Fig. 3.6b), an obvious decrease in turbidity was observed by $12 \%$. A 24-h turbidity was measured at 6.50 NTU, indicating an overall decrease by $44 \%$ (Fig. 3.6c).

The suspension of M-MIP particles causes the solution to appear turbid. The slow kinetics, indicated by the decrease in signal intensity (absorbance and turbidity) by $12-$ $13 \%$ for the first $4 \mathrm{~h}$ and a gradual decrease by additional $30 \%$ spanning $20 \mathrm{~h}$, demonstrated a reasonably steady suspension of M-MIP particles in aqueous medium. Low variation in particle kinetics is associated with the increased stability due to the formation of a colloidal suspension. This suspension-settlement pattern suggests minimal requirement of physical mixing process such as stirring or shaking. 


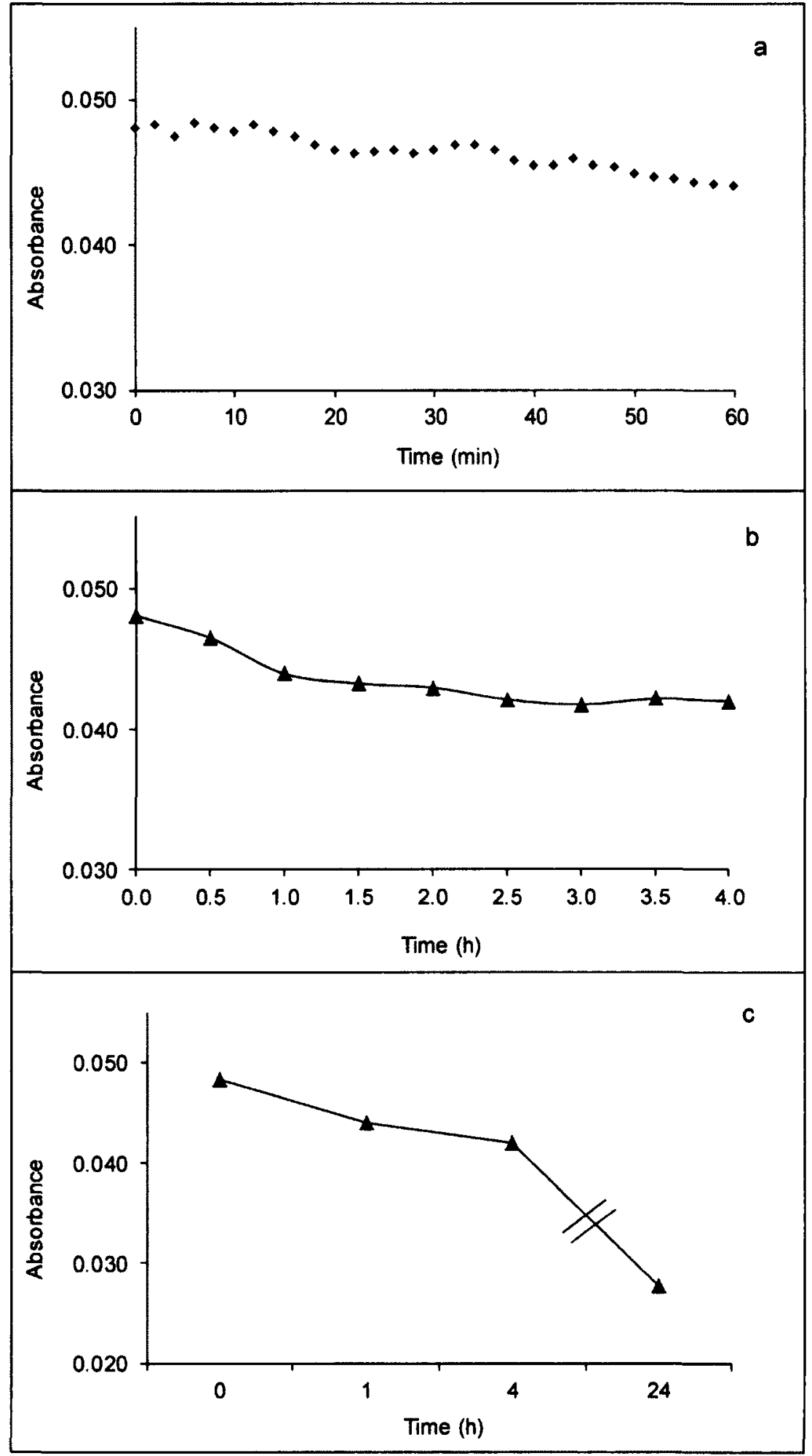

Fig. 3.5 M-MIP kinetic behavior (UV-Vis)

(initial concentration of M-MIP: $5.0 \mathrm{mg} / \mathrm{L}$ ) 


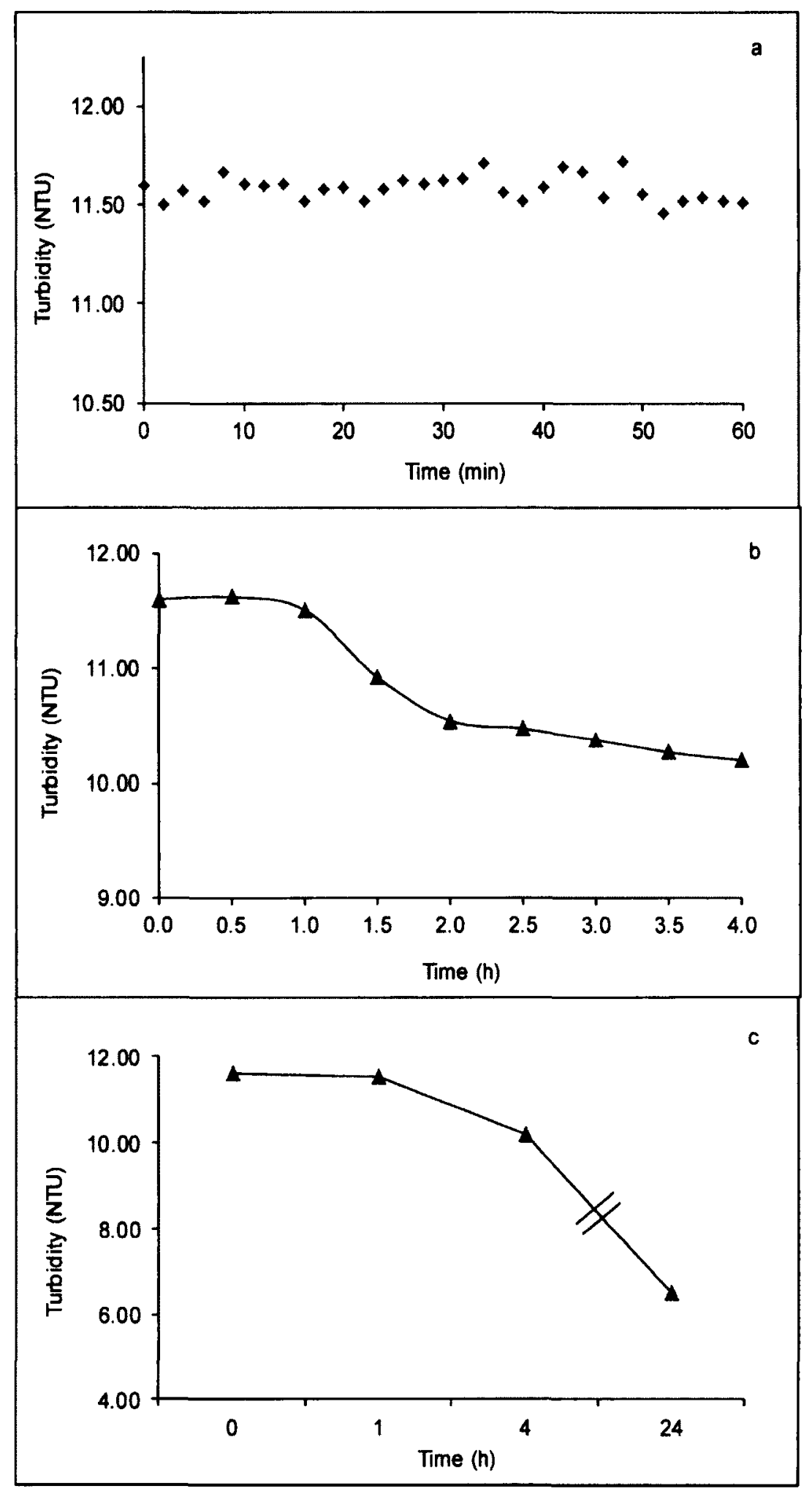

Fig. 3.6 M-MIP kinetic behavior (turbidity)

(initial concentration of M-MIP: $5.0 \mathrm{mg} / \mathrm{L}$ ) 


\subsubsection{Magnetic separation efficiency and comparison}

\subsubsection{Magnetic separation of M-MIP particles}

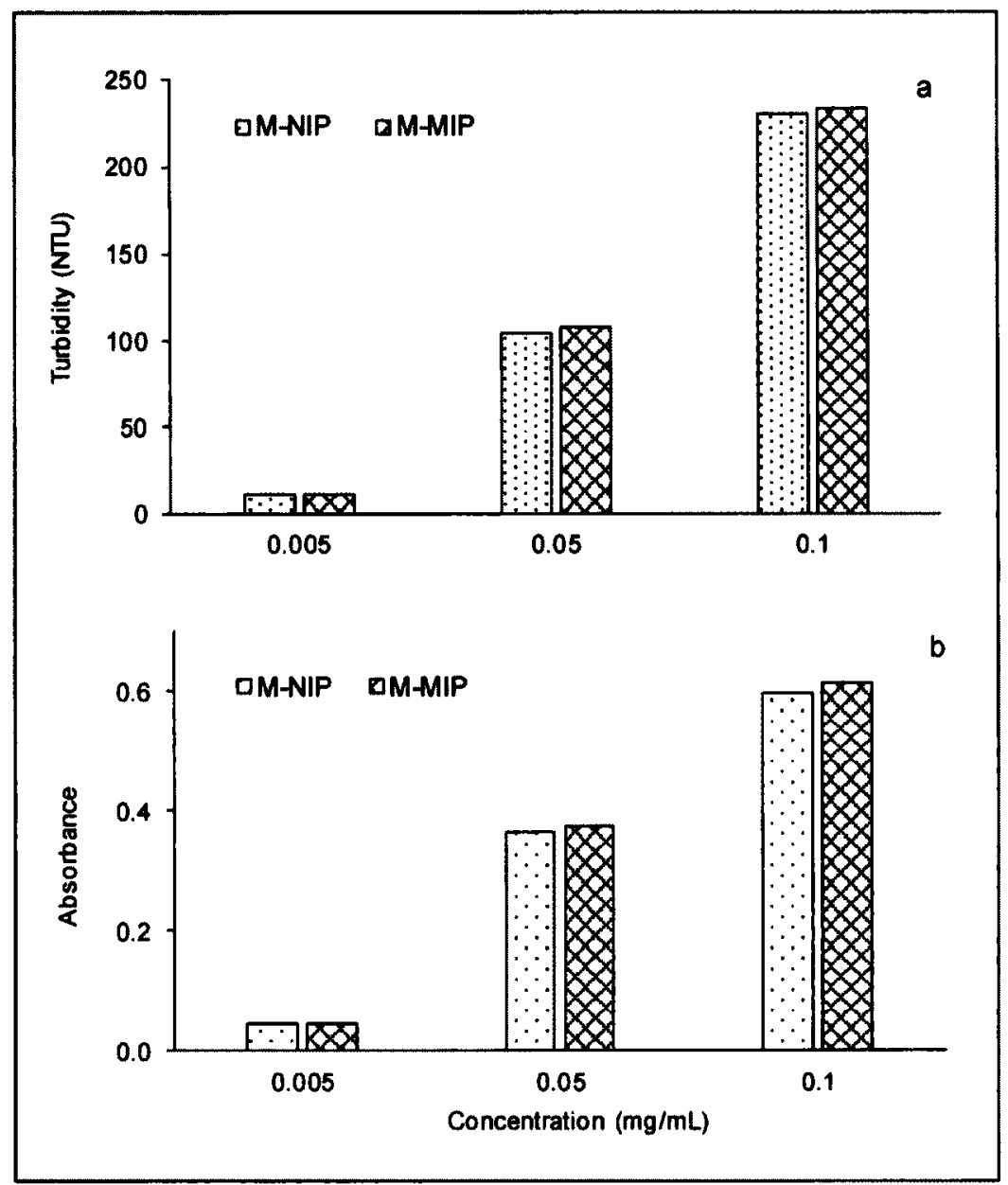

Fig. 3.7 Comparison of turbidity (a) and UV-Vis (b) measurements

In our previous work, the recovery of E2 M-MIP was determined with UV-Vis analysis. To secure a safe margin of complete particle recycling, turbidity measurement was introduced in analyzing residual content. As shown in Fig. 3.7, the variation in turbidity responses to the change in M-MIP and M-NIP concentration, which is comparable to UV-Vis absorbance. Therefore, UV-Vis analysis, in combination with turbidity measurement, is feasible for determining separation efficiency. A series of tests 
using this method showed that a full recovery of E2-BPA M-MIP particles was achieved in a range of $0.005-0.5 \mathrm{mg} / \mathrm{mL}$ in a $30-\mathrm{min}$ separation cycle, showing the effectiveness of magnetic separation.

\subsubsection{Centrifugation separation of M-MIP}

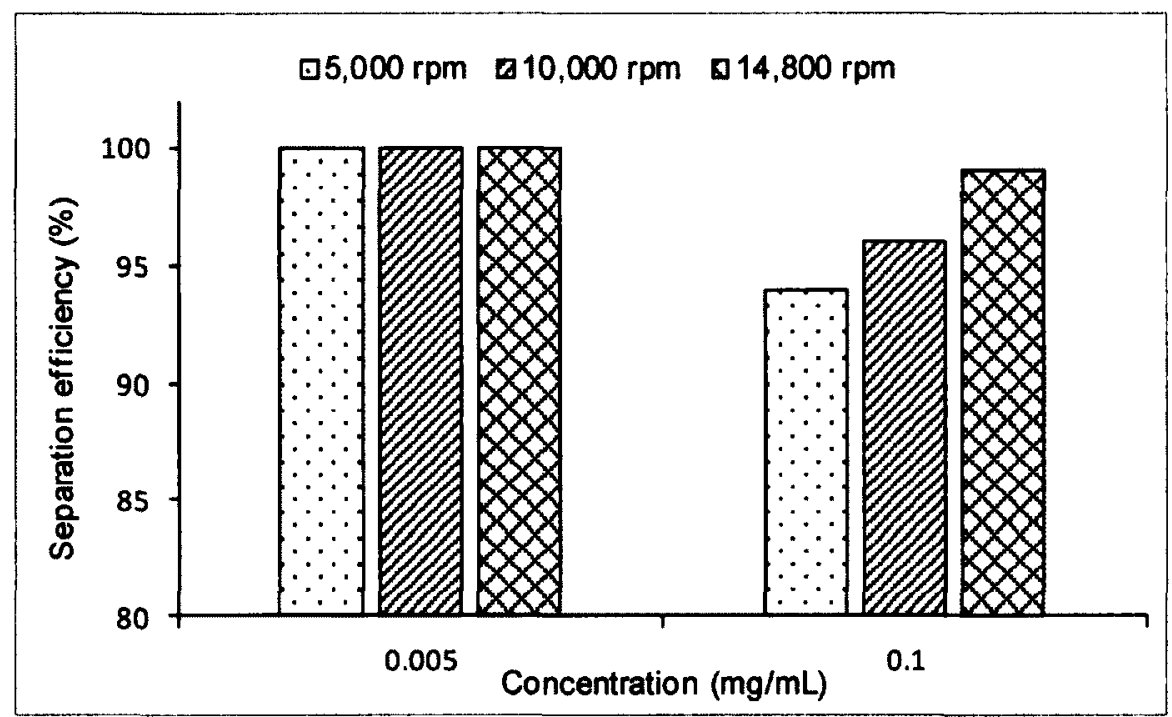

Fig. 3.8 Effect of centrifugation speed

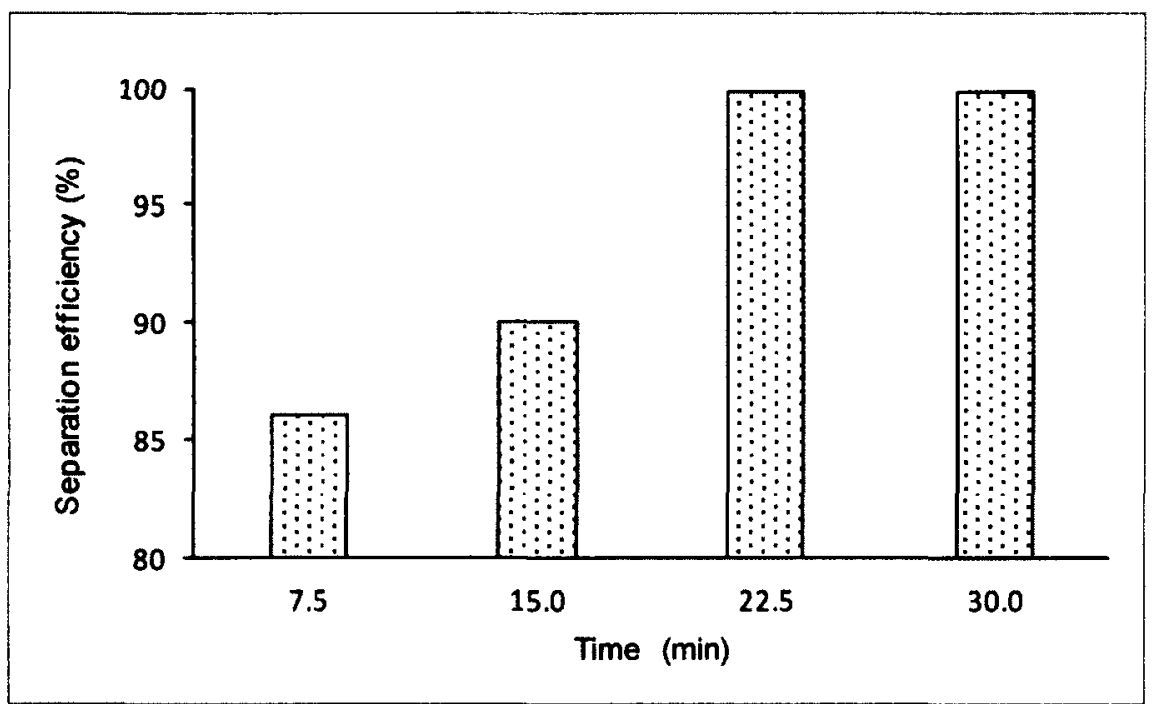

Fig. 3.9 Effect of centrifugation duration 
In this study, the magnetic recovery of M-MIP was further compared to the two commonly used separation techniques, centrifugation and membrane filtration.

Different centrifugation speeds $(5,000,10,000$ and $14,800 \mathrm{rpm})$ and durations were examined for aqueous M-MIP suspensions at various contents. In general, higher recovery was obtained for lower M-MIP content at faster centrifugation. At a low level (0.005 $\mathrm{mg} / \mathrm{mL})$, satisfactory recoveries were achieved at all three speeds. However, a range of recoveries from $94 \%$ to $99 \%$ was observed for M-MIP suspension at $0.1 \mathrm{mg} / \mathrm{mL}$ (Fig. 3.8). The recovery of M-MIP was significantly dependent on centrifugation duration (Fig. 3.9). For a range of durations, 7.5, 15.0, 22.5 and $30.0 \mathrm{~min},>90 \%$ recovery for an $0.1 \mathrm{mg} / \mathrm{mL}$ of M-MIP suspension was obtained only at or above $10,000 \mathrm{rpm}$. Therefore, to recover $0.1 \mathrm{mg} / \mathrm{mL}$ of M-MIP from aqueous solution, a minimum of $30 \mathrm{~min}$ at 10,000 rpm was required for a $>95 \%$ recovery. The quantitative recovery of M-MIP was also challenged by separating the settled solid from solution after centrifugation, indicated by a high variation in UV-Vis absorbance.

\subsubsection{Membrane filtration of M-MIP}

Commercially obtained syringe filter incorporated with polyethersulfone (PES) membrane (0.22 $\mathrm{\mu m}$ pore size) was employed for comparing solid-liquid separation efficiency. The allowable amount of M-MIP that can be filtered through the membrane for an acceptable flow rate was optimized. A constant flow of $0.5 \mathrm{~mL} / \mathrm{min}$ appeared to be suitable for a 5-mL M-MIP suspension at the studied concentrations. PES membrane filter was able to remove M-MIP to a satisfactory level, indicated by 0.00 NTU of residual turbidity after filtering M-MIP suspensions in a M-MIP range of 0.005 to $0.5 \mathrm{mg} / \mathrm{mL}$. However, clogging with high back pressure was encountered for higher sample loading and higher particles content as a result of the accumulation of particles on the membrane. Relatively poor filterability was a problem, as commonly found with 
membrane filtration practice [34]. PES membrane filtration shows higher recovery over centrifugation, and comparable to magnetic separation. However, it is not satisfied from production perspective for a steady separation of nanoparticles due to the pore-size determined membrane permeability. Particle reusability is not favored as well by this separation technique.

The results demonstrate that magnetic separation is advantageous over membrane filtration and centrifugation for the separation of M-MIP nanoparticles, appearing to be a promising technique for a steady, cost-effective M-MIP recovery.

\subsubsection{LC-MS/MS analysis}

\subsubsection{LC-MS/MS optimization}

A LC-MS/MS analytical method was developed for the determination of five EDCs (E1, E2, E3, BPA and DES). MS/MS was performed using electrospray ionization (ESI) probe in negative polarity (ESI-negative) with reference to the recent studies, in which ESI-negative was found to be suitable for EDCs identification and quantitation [35-37]. Using an ESI-negative steroid and phenolic EDCs give exclusively deprotonated molecular ions $[\mathrm{M}-\mathrm{H}]^{-}$and abundant product ions. MS/MS compound-dependent parameters were optimized by infusing individual standards at $100 \mathrm{ng} / \mathrm{mL}$. MS/MS spectra were acquired to identify the precursor-product ion transition for each compound. The ion source parameters were chosen by direct infusion of single compound and further tuned up with stepwise optimization at $380{ }^{\circ} \mathrm{C}$ with $-4500 \mathrm{~V}$ ionspray voltage.

The chromatographic separation of five EDCs was examined using a $100 \mathrm{ng} / \mathrm{mL}$ standard mixture for optimum LC parameters. Ultimately, a 25-min cycle with an 18-min non-linear gradient from $10 \%$ to $90 \%$ acetonitrile in water and a 7 -min re-equilibration at a flow rate of $0.3 \mathrm{~mL} / \mathrm{min}$ was established to separate five EDCs at ambient temperature. 


\subsubsection{Quantitative measurement}

LC-MS/MS quantitative analysis was performed in multiple reaction monitoring (MRM) mode to maximize sensitivity. The precursor ion used for MRM was the corresponding deprotonated molecular ion. The most intense product ion was paired with the precursor ion for quantitation. The assay of EDCs had the limits of detection of $0.5 \mathrm{ng} / \mathrm{mL}$ for E1, E2, E3 and BPA, and $0.6 \mathrm{ng} / \mathrm{mL}$ for DES (calculated as a signal to noise ratio of 3). The obtained calibration curves exhibited good linearity with the regression coefficients ( $R^{2}$ values) greater than 0.999 for five EDCs. The EDCs residual in the supernatant was measured after magnetic separation at the end of binding. Binding efficiency or capacity was calculated using the LC-MS/MS data acquired with the optimized conditions.

The analytical challenge of measuring EDCs in aqueous environment has been a major research focus due to the high estrogenic effects of EDCs at very low concentration (ng/L) levels. For instance, the occurrence of E1 and E2 in the final effluent from sewage treatment plants was reported at concentrations in the tens of $\mathrm{ng} / \mathrm{L}$ range that are sufficient to cause adverse effects on aquatic living organisms [38]. From an analytical perspective, such low concentrations call for higher sensitive and selective analytical methods for their reliable detection. LC-MS/MS is found most suitable for EDCs determination at environmentally relevant concentrations since this technique provides high sensitivity and selectivity for the unambiguous identification and quantification of environmental pollutants at trace-level concentrations even in complex matrices [39-41]. This study investigated the effectiveness of LC-MS/MS for the simultaneous determination of steroid and phenolic EDCs without sample preparation such as derivatization and pre-concentration. The method provides enough sensitivity to analyze EDCs for the research purpose. 


\subsubsection{Effect of environmental variables on adsorption efficiency}

\subsubsection{Effect of ionic strength}

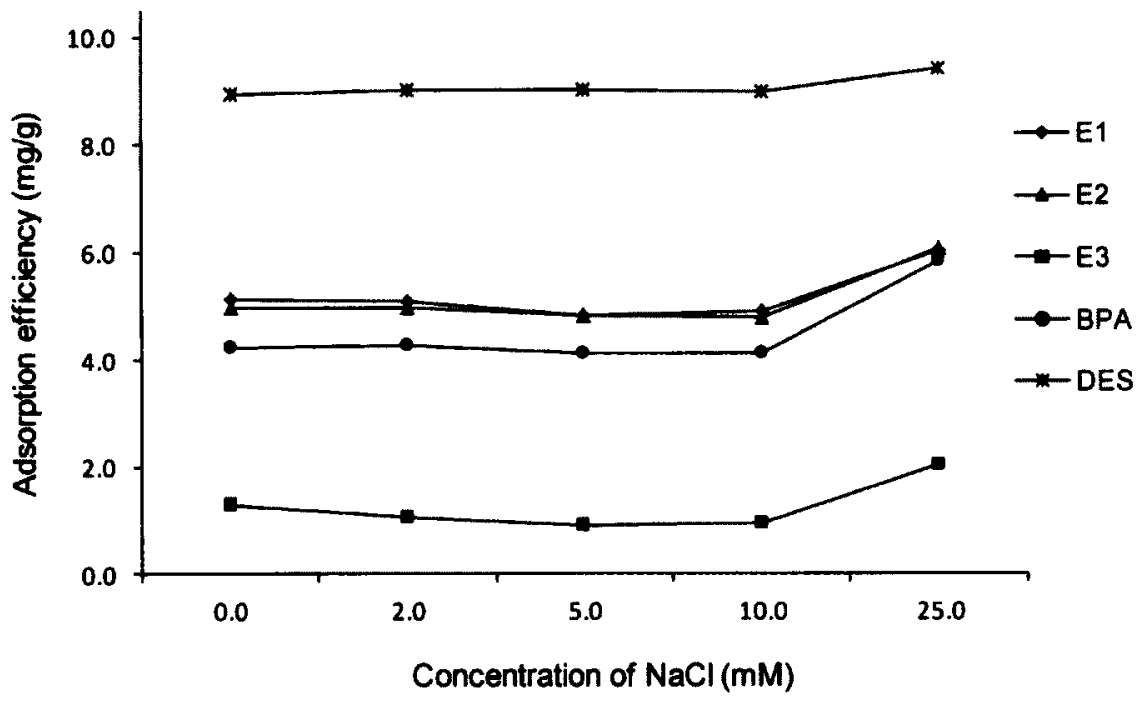

Fig. 3.10 Effect of ionic strength

Aqueous $\mathrm{NaCl}$ solutions were used to assess the influence of ionic strength on adsorption efficiency. As shown in Fig. 3.10, it is obvious that the adsorption capacity, expressed as the adsorbed amount ( $\mathrm{mg}$ ) by per gram of M-MIP, was not affected in the presence of $\mathrm{NaCl}$ at a low concentration range from 2.0 to $10.0 \mathrm{mM}$. However, an increased adsorption was noted for all the studied EDCs at a higher $\mathrm{NaCl}$ content $(25.0$ $\mathrm{mM}$ ). The obtained results were in consistence with the previous observations on steroid and phenolic EDCs $[17,19]$. Hydrophobic interactions could be enhanced by the addition of salt due to the salting-out effect [42]. At low salt concentrations, salting-out effect overcomes the influence of electrostatic screening effect, resulting in an almost constant adsorption capacity [43]. The minor effect of low ionic strength suggests the potential of applying M-MIP to natural waters, in which inorganic salts are commonly found. 


\subsubsection{Effect of humic acid}

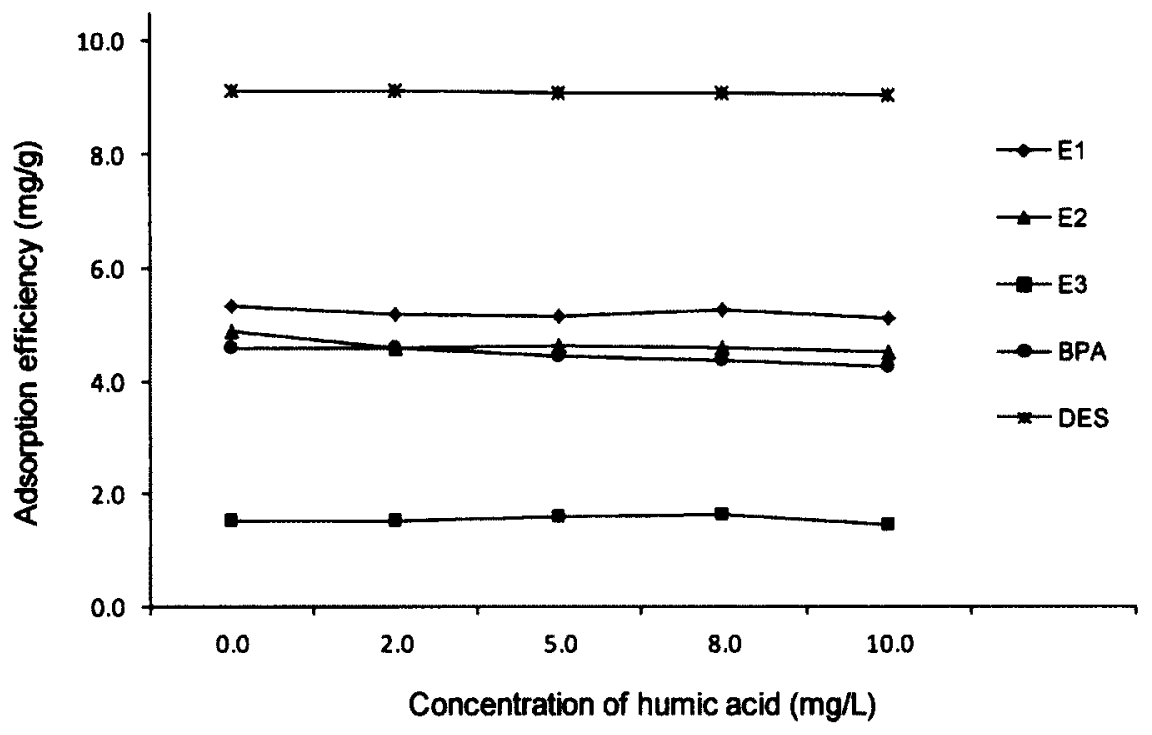

Fig. 3.11 Effect of HA

The physical state and environmental fate of organic pollutants are complicated by the presence of humic acid, a complex containing carboxyl and phenolate groups in the aquatic environment [44]. In this work, artificial aqueous solutions containing 2.0 to $10.0 \mathrm{mg} / \mathrm{L}$ of HA were prepared and equilibrated with M-MIP to evaluate the influence of HA. The results (Fig. 3.11) show that the effectiveness of binding interactions was not affected by the presence of $\mathrm{HA}$ up to $10.0 \mathrm{mg} / \mathrm{L}$. As a consequence, the negative effect of HA on selective and efficient removal of target EDCs, arising from the interfering interactions of EDCs-HA and M-MIP-HA, became less important, implying the applicability of M-MIP to the real water streams. 


\subsubsection{Effect of $\mathrm{pH}$}

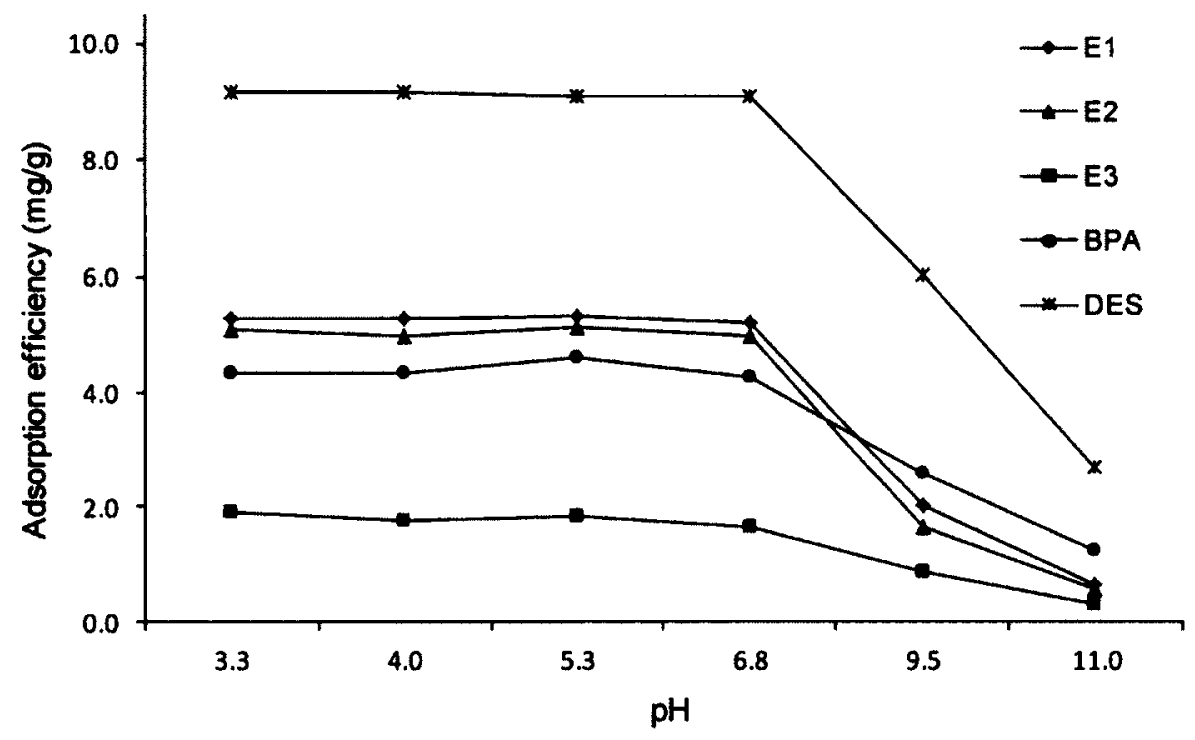

Fig. 3.12 Effect of pH

$\mathrm{pH}$ is one of the most influential variables that can affect adsorption performance [45]. For this reason, pH effect was investigated. Fig. 3.12 presents a common and distinct feature of $\mathrm{pH}$ effect in a wide range of 3.3 to 11.0. A negligible influence in weakly acidic, neutral to weakly alkaline conditions was observed. However, the reduction in the adsorption of EDCs started at $\mathrm{pH}$ values higher than 9 and decreased dramatically with the increasing $\mathrm{pH}$ in alkaline condition. Steroid and phenolic EDCs were found to be affected in the same way. The adsorption behaviors were associated with the highly weak acidic character of EDCs. pKa values are between 10.3 and 10.4 for E1, E2, E3 and BPA [46, 47]. Therefore, they undergo ionization in alkaline condition. At $\mathrm{pH} 11.0$, the difference was the highest for DES that has a pKa value of 9.7 [48]. The result was in agreement with the humic acid experiment, in which a steady adsorption was found in a weakly acidified solution. The findings have its significance since both the 
adsorbent (M-MIP) and the adsorbate (EDCs) contain functional groups that can be affected by the matrix acidity or alkalinity.

\subsubsection{Effect of temperature}

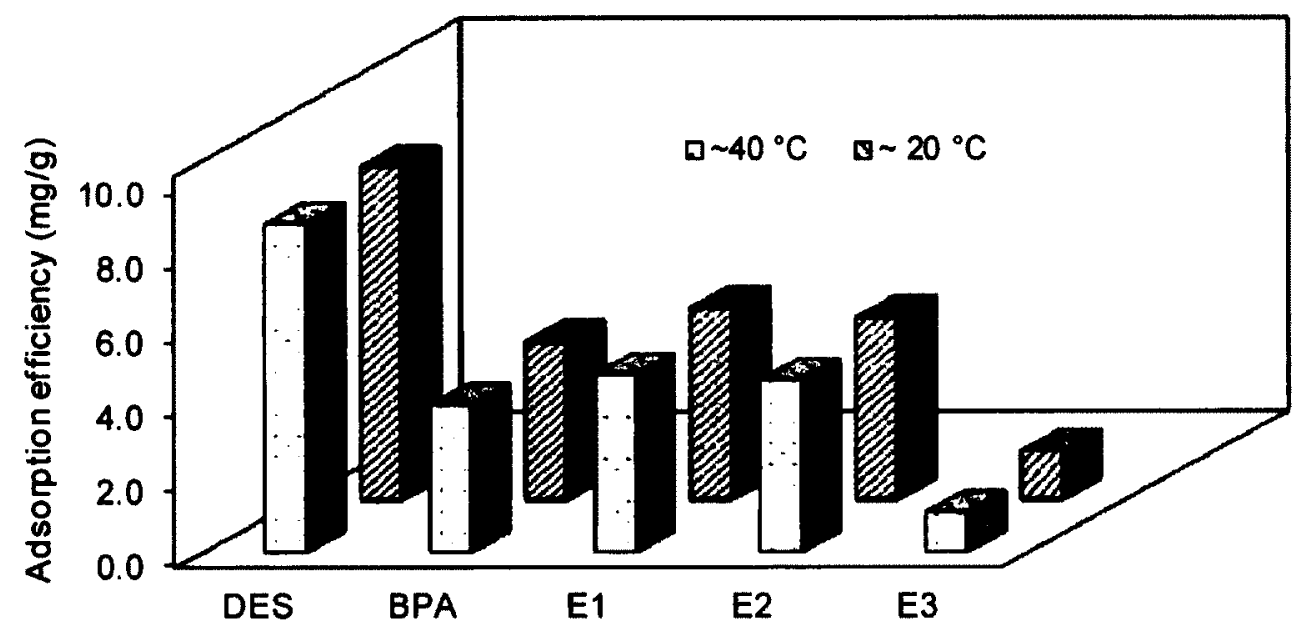

Fig. 3.13 Effect of temperature

Temperature plays its role in the target-adsorbent interactions. Thermo-sensitive polymers can change the dimensions of their structure depending on temperature stimulation from the environment [49]. Temperature effect testes were carried out at ambient $\left(20 \pm 0.5^{\circ} \mathrm{C}\right)$ and a reasonable higher temperature at $40 \pm 0.5^{\circ} \mathrm{C}$. The adsorption efficiency is presented in Fig. 3.13. It can be seen that when temperature rose from 20 ${ }^{\circ} \mathrm{C}$ to $40{ }^{\circ} \mathrm{C}$, the uptake of EDCs by M-MIP decreased slightly. A relatively higher temperature may increase the solubility of EDCs and strengthen the desorption process [50], resulting in a slight adsorption reduction. However, a limited data obtained in this experiment does not allow for concluding a general trend on temperature effect due to the complexity of actual adsorption-desorption practice. 


\subsubsection{Class-specific adsorption}

\subsubsection{Equilibrium adsorption}

Aqueous solutions containing $0.25-4.00 \mu \mathrm{g} / \mathrm{mL}$ EDCs were equilibrated with MMIP (M-NIP) for $6 \mathrm{~h}$ to study the adsorption-desorption performance. The equilibrium isotherms are presented in Fig. 3.14. The Freundlich model, represented by the following equation, was applied to describe the adsorption behavior.

$$
\log Q_{e}=\log K_{f}+(1 / n) \log C_{e}
$$

Where $Q_{\theta}$ is the equilibrium adsorption capacity of adsorbent toward targets $(\mathrm{mg} / \mathrm{g}), C_{e}$ is the equilibrium concentration of targets in solution $(\mathrm{mg} / \mathrm{L}) . \mathrm{K}_{\mathrm{f}}$ and $1 / \mathrm{n}$ are the Freundlich constants that are related to the adsorption capacity and adsorption intensity of adsorbent. The Freundlich isotherm model was employed for its accuracy and applicability at low concentrations.

The Freundlich isotherm model parameters are summarized in Table 3.1. The experimental data were well fitted to Freundlich model. The intensity parameters $(1 / n)$ for EDCs are less than 1.0, representing the favorable uptake of EDCs by M-MIP. The good agreement of experimental data with Freundlich model suggests a heterogeneous imprinted surface since MIPs are commonly characterized by a high degree of binding site heterogeneity [51]. The lower $1 / \mathrm{n}$ values indicate a more heterogeneous property of M-MIP than that of M-NIP, attributed to the formation of imprinted sites on polymer surface. High regression coefficient values were obtained by applying Freundlich model for all the studied EDCs. The high Freundlich constant $K_{f}$ suggests that M-MIP adsorption capacity is less sensitive to the increase in target concentration. 


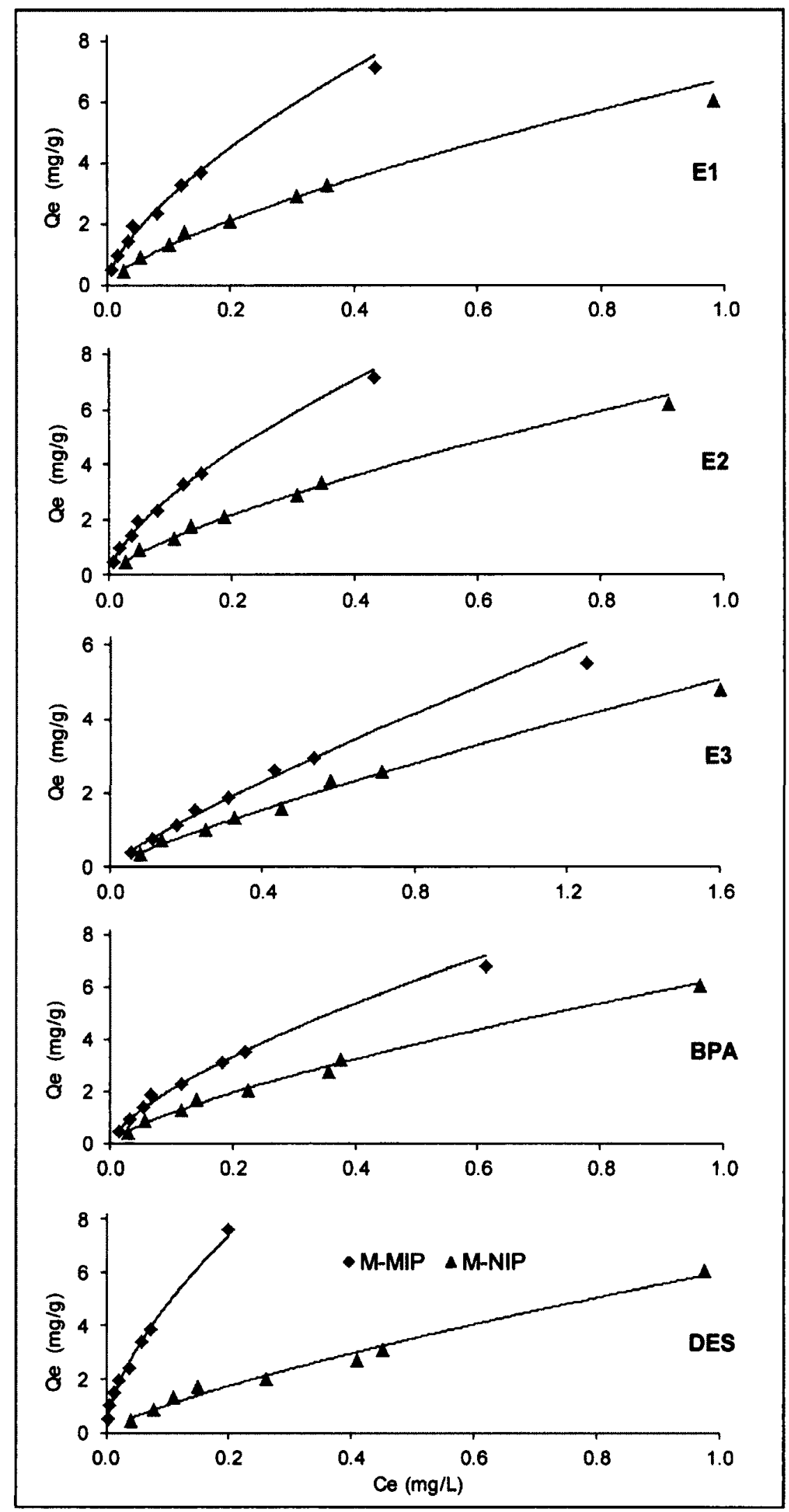

Fig. 3.14 Equilibrium adsorption ( $6 \mathrm{~h}$ incubation at ambient temperature) 
Table 3.1 Freundlich isotherm parameters

\begin{tabular}{c|c|c|c|c|c}
\hline Adsorbate & Adsorbent & $\mathrm{K}_{\mathrm{f}}$ & $1 / \mathrm{n}$ & $\mathrm{n}$ & $\mathrm{R}^{2}$ \\
\hline E1 & M-MIP & 13.08 & 0.66 & 1.51 & 0.987 \\
& M-NIP & 6.74 & 0.72 & 1.39 & 0.987 \\
\hline E2 & M-MIP & 12.96 & 0.66 & 1.52 & 0.994 \\
& M-NIP & 6.99 & 0.73 & 1.37 & 0.990 \\
\hline E3 & M-MIP & 5.02 & 0.85 & 1.18 & 0.993 \\
& M-NIP & 3.40 & 0.86 & 1.17 & 0.987 \\
\hline BPA & M-MIP & 10.05 & 0.68 & 1.46 & 0.991 \\
& M-NIP & 6.34 & 0.73 & 1.38 & 0.989 \\
\hline DES & M-MIP & 19.98 & 0.62 & 1.62 & 0.987 \\
& M-NIP & 6.00 & 0.77 & 1.30 & 0.976 \\
\hline
\end{tabular}

From an equilibrium perspective, E2-BPA imprinted M-MIP has the adsorption capacities toward EDCs in an order of DES > E2, E1 > BPA > E3. M-MIP exhibits an average of $7-20 \%$ higher equilibrium binding capacity than M-NIP. The difference increases with the increased EDCs concentration, showing the non-specific binding property of M-NIP.

\subsubsection{Adsorption capacity of M-MIP to templates and EDCs}

Fig. 3.15 exhibits the adsorption difference using E2-BPA M-MIP to rebind the templates and a group of EDCs. In the absence of other EDCs, M-MIP had adsorption capacity of 6.0 and $5.8 \mathrm{mg} / \mathrm{g}$ toward E2 and BPA. The uptake of E1, E2, E3, BPA and EDCs by M-MIP from a five EDCs mixture $(0.5 \mu \mathrm{g} / \mathrm{ml}$ for each compound) was $5.0,5.0$, $1.0,4.7$ and $9.1 \mathrm{mg} / \mathrm{g}$, respectively. There was a decrease in adsorption capacity by $17 \%$ for E2 and $19 \%$ for BPA. However, the decreased adsorption capacities for E2 and BPA were leveled off by a significant increase in total adsorption capacity for five EDCs, indicating a satisfactory group removal ability of M-MIP. 


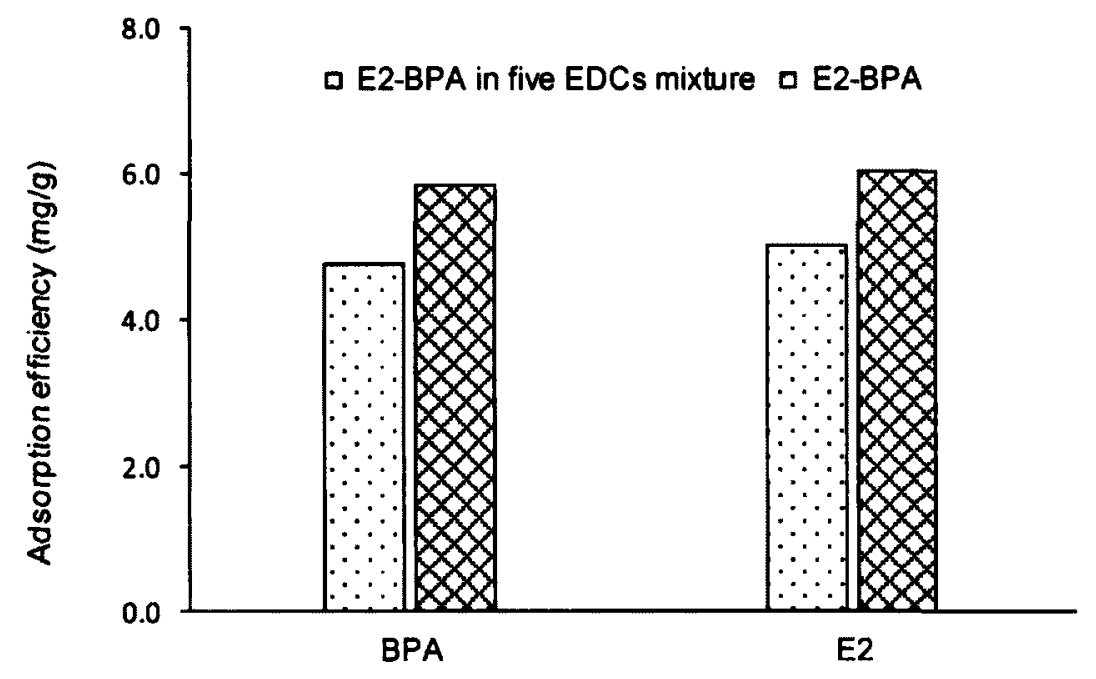

Fig. 3.15 Comparison of adsorption efficiency with co-existing EDCs

\subsubsection{Selective adsorption of EDCs}

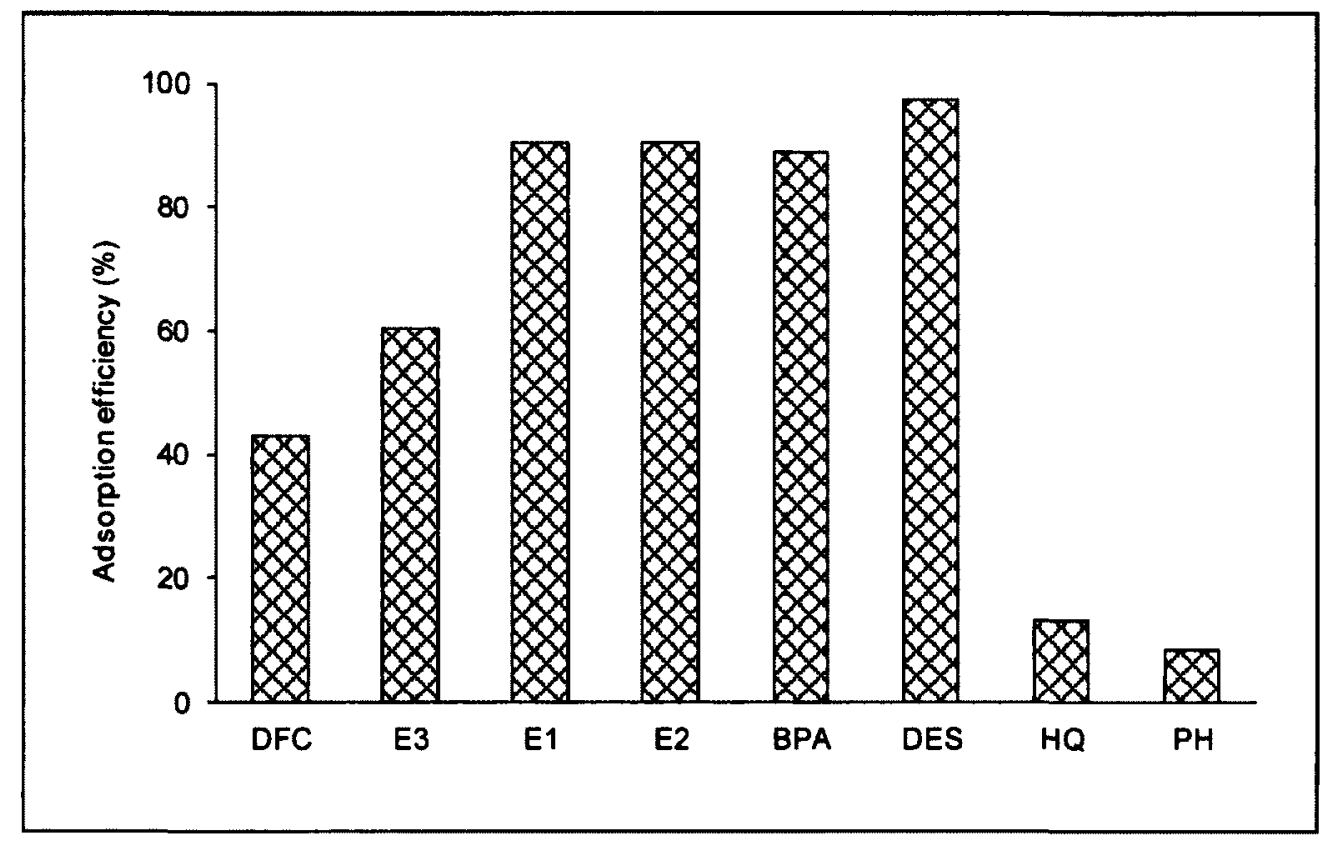

Fig. 3.16 Adsorption efficiency toward EDCs, DFC, HQ and PH 
As shown in Fig. 3.16, M-MIP has higher binding capacities toward EDCs than HQ, PH and DFC. The adsorption efficiency was found at $90 \%, 91 \%, 60 \%, 89 \%$ and $97 \%$ for $E 1, E 2, E 3, B P A$ and DES in the presence of the interfering compounds. Low binding capacities were observed for DFC (43\%), HQ (13\%) and PH (8\%). Within the studied compounds, E1 and E3 are structurally similar to E2. So is DES to BPA. However, there is significant difference between the EDCs and the interfering species in structure and molecular configuration. Despite the fact that DFC, HQ and PH contain carboxyl or hydroxyl functional groups capable of hydrogen bonding, E2-BPA M-MIP exhibits recognition when competing species are present. The binding characteristics presents a similar adsorption performance to those using E2 imprinted MIP for steroid [19] and BPA imprinted MIP for phenolic EDCs [17], demonstrating that E2-BPA M-MIP allows for a selective extraction of low level of EDCs in the presence of potentially interfering components. Hence, E2-BPA M-MIP could be a promising artificial sorbent for the classspecific removal of EDCs.

\subsubsection{Application of M-MIP to environmental samples}

\subsubsection{Effect of sample matrix}

The matrix effect of real water samples from different sources was examined. Tap water (TW) and river water (RW) samples were spiked with EDCs $(1000 \mu \mathrm{g} / \mathrm{L}$, final) for a 2-mL sample loading, incubated with $1.0 \mathrm{mg}$ of adsorbent (M-MIP or M-NIP) to assess the practicability and applicability. Tap water was sampled from our lab. It had a pH of 5.5 and a turbidity of 0.00 NTU. River water samples, collected from the Ottawa River (Bate Island, Ottawa, Canada), had a pH of 6.9 and a turbidity of 2.14 NTU. DDW sample (pH 5.5 and turbidity $0.00 \mathrm{NTU}$ ) was used for comparison. All samples were treated without pre-filtration to maintain their original parameters. 


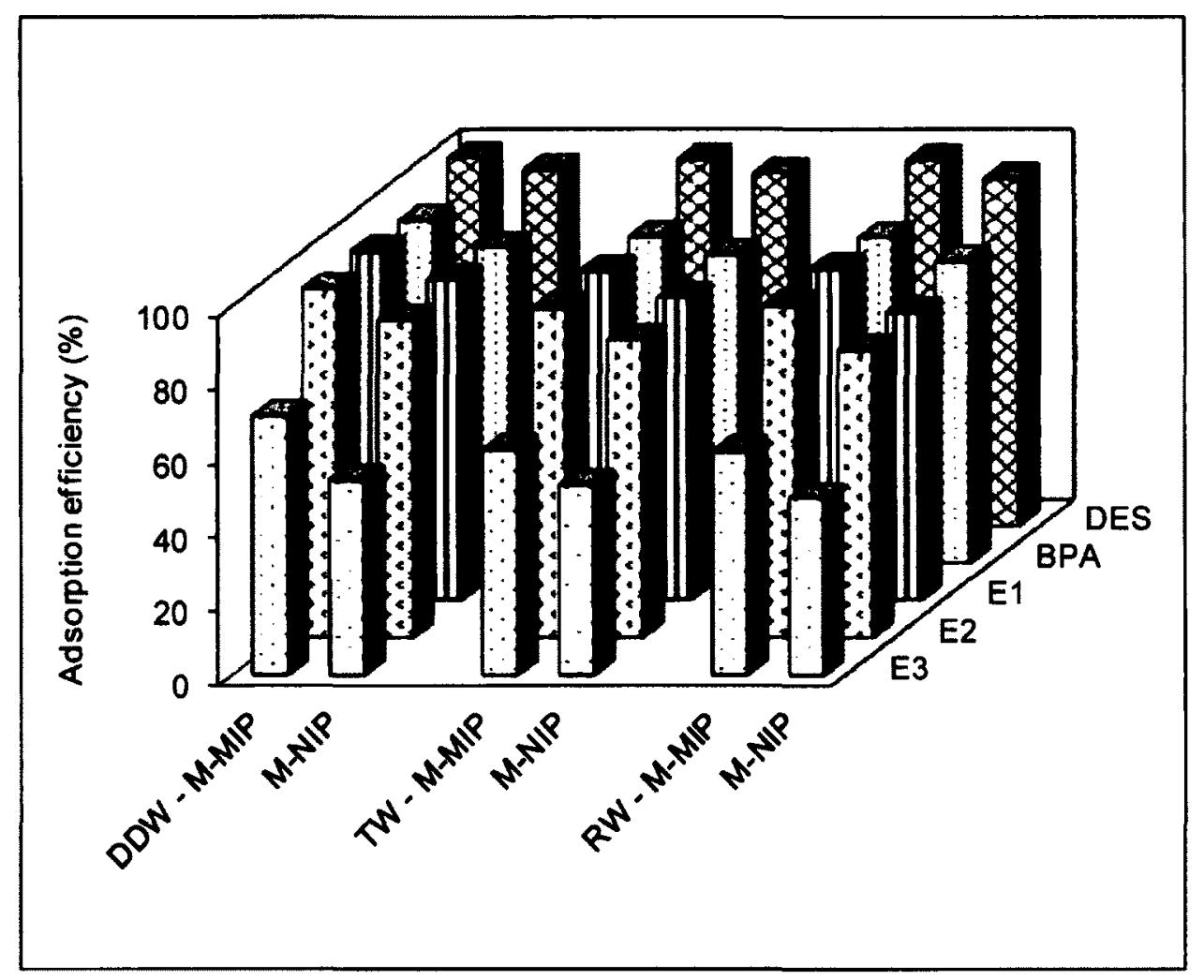

Fig. 3.17 Recovery of EDCs from spiked aqueous samples

(initial concentration of EDCs: $1.0 \mu \mathrm{g} / \mathrm{mL} ; 0.5 \mathrm{mg} / \mathrm{mL}$ adsorbent, $1 \mathrm{~h}$ incubation)

The recoveries were calculated at $90 \%, 90 \%, 61 \%, 88 \%$ and $98 \%$ for E1, E2, E3, BPA and DES from the spiked river water sample (Fig. 3.17). Compared to the spiked DDW sample, the decrease in adsorption capacity was found at $1 \%$ for DES, an average of $4-5 \%$ for E1, E2, BPA and $10 \%$ for E3. No significant difference was observed in the extraction of EDCs using M-MIP from the spiked river water and tap water samples. However, the uptake of EDCs by M-NIP presents a different pattern. A range of $1-6 \%$ decrease in adsorption capacity for the five EDCs was observed from the DDW sample to tap water sample. An additional decrease by $2-4 \%$ was found from the tap water to river water sample, showing that M-NIP adsorption efficiency decreases with the increased complexity of sample matrix. The river water sample had a high turbidity value 
of $>1.0$ NTU. The pale greenish color indicated the presence of organic substances. The findings reveal that M-MIP recognition ability was not affected dramatically by the environmental matters. The results further confirm the importance of a sufficient adsorbent loading for a satisfactory adsorbate removal.

\subsubsection{Simultaneous removal of EDCs}

Finally, M-MIP was tested for the effectiveness in removing EDCs at a trace concentration level with a larger real water sample loading. The recoveries of five EDCs from 100-mL river water samples spiked at $10 \mu \mathrm{g} / \mathrm{L}$ of EDCs and incubated with $50 \mathrm{mg}$ of M-MIP were performed using the binding and separation procedures described in this research. E1, E2, E3, BPA and DES were recovered to a satisfactory degree $(100 \pm 1 \%)$. Therefore, the simultaneous removal of selected EDCs using M-MIP appears to be a promising alternative in EDCs associated pollution control.

\subsubsection{Reuse and regeneration potential}

MIPs can be re-used in practical applications [52]. The regenerating property of MIPs is often considered to have a great influence on extending their utilization [53].

In this work, M-MIP was repeatedly used for rebinding the templates, E2 and BPA, by a simple organic solvent washing procedure after each adsorption to analyze their regenerating property. A 7-cycle reuse investigation showed that satisfactory parameters of regeneration and stability were obtained. Fig. 3.18 presents the reuse and regeneration potential of M-MIP. M-MIP adsorbs the templates with a good rebinding capacity after regenerations. In addition, M-MIP performed well for the adsorption of a group of EDCs. 


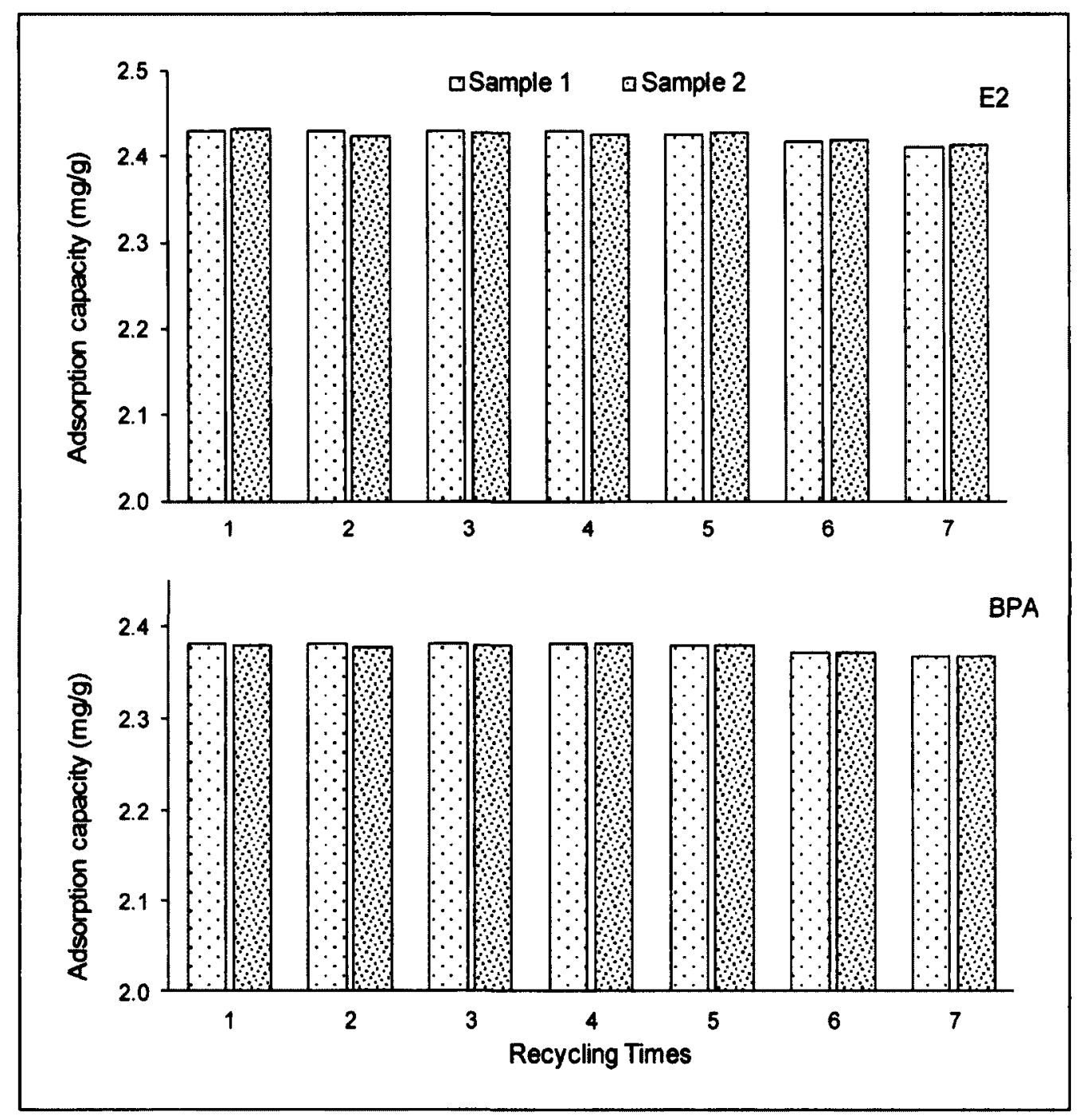

Fig. 3.18 Reuse and regeneration potential of M-MIP

Fig. 3.19 displays the adsorption efficiency using the regenerated M-MIP to bind EDCs after seventh use. The reduction in adsorption capacities was less than $2 \%$ for the five EDCs compared to the M-MIP freshly prepared. The results show that molecular recognition property of M-MIP remains after regenerations. Taking into account the fact that all the separation process in the reuse and regeneration experiment was performed under the same magnetic strength and separation duration, magnetic properties remain without obvious deterioration. Previous study demonstrated that magnetic MIP beads 
can be reused for at least 5 times without significant loss of magnetic moment and signal intensity [54]. Therefore, application of M-MIP to remove EDCs from waters shows potential to reduce the cost, offering an alternative in future treatment of water sources that are contaminated with EDCs.

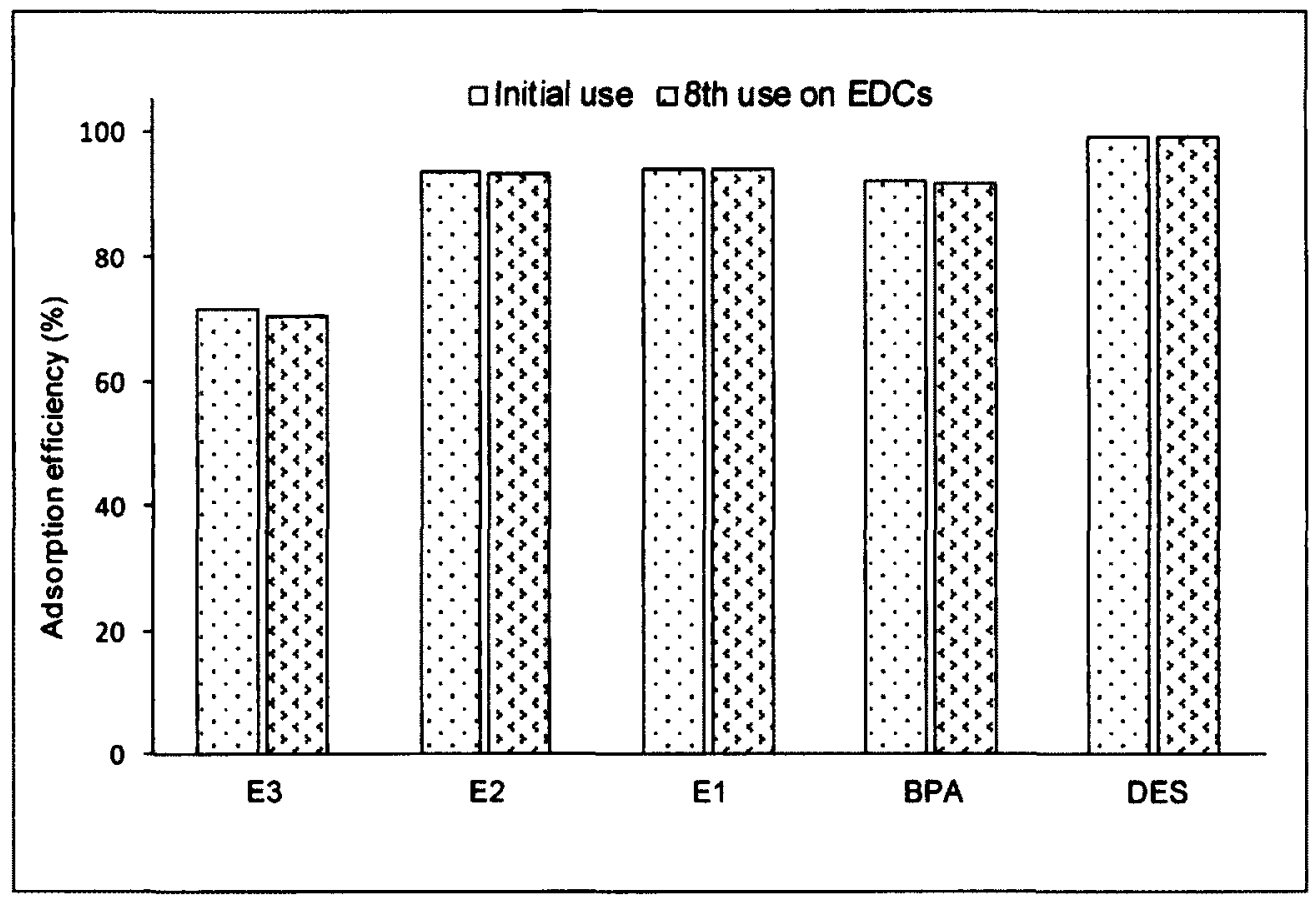

Fig. 3.19 Reuse of M-MIP for EDCs removal

\subsection{Conclusions}

The model steroid and phenolic EDCs imprinted polymer was encapsulated on magnetic nanoparticles for the generation of core-shell MIP by ultrasonication assisted synthesis. E2-BPA MIP exhibited strong magnetic response to facilitate an effective magnetic separation. The recognition ability and adsorption capacity toward the template molecules were high. The M-MIP possesses high selective affinity toward the selected EDCs that are structurally related to the templates. The minor effect of environmental 
and interfering factors demonstrated the reliability and applicability of the M-MIP to the complex environmental matrices. The M-MIP has regeneration potential for reuse at least seven times without significant deterioration in adsorption and magnetic properties.

The simultaneous removal of five representative steroid and phenolic EDCs from environmental samples was achieved at a trace $(10 \mu \mathrm{g} / \mathrm{L})$ concentration level. The research work suggested that multiple-template imprinted MIPs can be potentially utilized for an efficient and selective removal of classes of EDCs from aqueous matrices. 


\section{References}

1. C.G. Daughton, J. Am. Soc. Mass Spectrom., 12, 1067 (2001).

2. E.J. Routledge, J.P. Sumpter, Environ. Toxicol. Chem., 15, 241 (1996).

3. M. Hill, C. Stabile, K. Steffen, A. Hill, Environ. Pollut., 117, 295 (2002).

4. L.J. Mills, C. Chichester, Sci. Total Environ., 343, 1 (2005).

5. J. Ashby, Environ. Toxicol. Phar., 3, 87 (1997).

6. M. Clara, B. Strenn, N. Kreuzinger, Water Res., 38, 947 (2004).

7. C.G. Campbell, S.E. Borglin, F.B. Green, A. Grayson, E. Wozei, W.T. Stringfellow, Chemosphere, 65, 1265 (2006).

8. V. Matozzo, F. Gagné, M.G. Marin, F. Ricciardi, C. Blaise, Environ. Int., 34, 531 (2008).

9. M. Petrović, M. Solé, M.J. López de Alda, D. Barceló, Environ. Toxicol. Chem., 21, 2146 (2002).

10. S.S. Madsen, S. Skovbolling, C. Nielsen, B. Korsgaard, Aquat. Toxicol., 68, 109 (2004).

11. G. Ying, B.Williams, R. Kookana. Environ. Int., 28, 215 (2002).

12. V. Pacáková, L. Loukotková, Z. Bosáková, K. Stulík, J. Separ. Sci., 32, 867 (2009).

13. M. Clara, N. Kreuzinger, B. Strenn, O. Gans, H. Kroiss, Water Res., 39, 97 (2005).

14. B.T.S. Bui, K. Haupt, Anal. Bioanal. Chem., 398, 2481 (2010).

15. M. Lasáková, P. Jandera, J. Separ. Sci., 32, 799 (2009).

16. G. Vasapollo, R.D. Sole, L. Mergola, M.R. Lazzoi, A. Scardino, S. Scorrano, G. Mele, Int. J. Mol. Sci., 12, 5908 (2011).

17. Y. Lin, Y. Shi, M. Jiang, Y. Jin, Y. Peng, B. Lu, K. Dai, Environ. Pollut., 153, 483 (2008).

18. E. Herrero-Hernández, R. Carabias-Martínez, E. Rodríguez-Gonzalo, Int. J. Mol. Sci., 12, 3322 (2011). 
19. Z. Zhang, J. Hu, Water Air Soil Poll., 210, 255 (2010).

20. J.C. Bravo, R.M. Garcinuño, P. Fernández, J.S. Durand, Anal. Bioanal. Chem., 38, 1039 (2007).

21. Z. Meng, W. Chen, A. Mulchandani, Environ. Sci. Technol., 39, 8958 (2005).

22. C. Durairaj, S.J. Kim, H.F. Edelhauser, J.C. Shah, U.B. Kompella, Invest. Ophthalmol. Vis. Sci., 50, 4887 (2009).

23. Y. Watabe, T. Kubo, T. Nishikawa, T. Fujita, K. Kaya, K. Hosoya, J. Chromatogr. A, 1120,252 (2006).

24. K. Sreenivasan, J. Appl. Polym. Sci., 82, 889 (2001).

25. R. Suedee, T. Srichana, T. Chuchome, U. Kongmark, J. Chromatogr. B, 811, 191 (2004).

26. R. Suedee, V. Seechamnanturakit, A. Suksuwan, B. Canyuk, Int. J. Mol. Sci., 9, 2333 (2008).

27. L. Chen, S. Xu, J. Li, Chem. Soc. Rev., 40, 2922 (2011).

28. X. Feás, J. A. Seijas, M. P. Vá zquez-Tato, P. Regal, A. Cepeda, C. Fente, Anal. Chim. Acta, 631, 237 (2009).

29. M. Jiang, J.H. Zhang, S.R. Mei, Y. Shi, L.J.Zou, Y.X. Zhu, K. Dai, B. Lu, J. Chromatogr. A., 1110, 27 (2006).

30. H. Sanbe, J. Haginaka J. Pharm. Biomed. Anal., 30, 1835 (2002).

31. B. Huang, F. Bai, X. Yang, W. Huang, Chinese J. Polym. Sci., 28, 277 (2010).

32. M. Khasanah, Mudasir, A. Kuncaka, E. Sugiharto, G. Supriyanto, S. Wafiroh, Indo. J. Chem., 10, 295 (2010).

33. J. Wang, H. Li, X. Yang, Polym. Adv. Technol., 20, 965 (2009).

34. H. Huang, K. Schwab, J.G. Jacangelo, Environ. Sci. Technol., 43, 3011 (2009).

35. M. L. Gross, Int. J. Mass Spectrom., 200, 611 (2000).

36. Y. Sun, C. Gu, X. Liu, W. Liang, P. Yao, J.L. Bolton, R.B. van Breemen, 
J. Am. Soc. Mass Spectrom., 16, 271 (2005).

37. E. Magi, C. Scapolla, M.D. Carro, C. Liscio, J. Mass. Spectrom., 45, 1003 (2010).

38. E.J. Routledge, D. Sheahan, C. Desbrow, G.C. Brighty, M. Waldock, J.P. Sumpter, Environ. Sci. Technol., 32, 1559 (1998).

39. C. Baronti, R. Curini, G. D'Ascenzo, A.Di Corcia, A. Gentili, R. Samperi, Environ. Sci. Technol., 34, 5059 (2000).

40. P. Adler, T. Steger-Hartmann, W. Kalbfuss, Acta Hydrochim. Hydrobiol., 29, 227 (2001).

41. M. Sole, M. Lopez de Alda, M. Castillo, C. Porte, K. Ladegaard-Pedersen, D. Barcelo, Environ. Sci. Technol., 34, 5076 (2000).

42. P. Janoš, H. Buchtová, M. Rýznarová, Water Res., 37, 4938 (2003).

43. Q. Yu, S. Deng, G. Yu, Water Res., 42, 3089 (2008).

44. P.F. Landrum, S.R. Nihart, B.J. Eadle, W.S. Gardner, Environ. Sci. Technol., 18, 187 (1984).

45. H. Kempe, M. Kempe, Anal. Chem., 78, 3659 (2006).

46. A.R. Hurwitz, S.T. Liu, J. Pharm. Sci., 66, 624 (1977).

47. M. Clara, B. Strenn, E. Saracevic, N. Kreuzinger, Chemosphere, 56, 843 (2004).

48. J. Zhou, C. Hu, R. Zhao, Anal. Methods, 3, 2568 (2011).

49. W. Zhang, X. He, W. Li, Y. Zhang, Chem. Commun., 48, 1757 (2012).

50. A.H. Mollah and C.W. Robinson, Water Res., 30, 2901(1996).

51. S. Wei, B. Mizaikoff, Biosens. Bioelectron., 23, 201 (2007).

52. J. Zhang, M. Jiang, L. Zou, D. Shi, S. Mei, Y. Zhu, Y. Shi, K. Dai, B. Lu, Anal. Bioanal. Chem., 385, 780 (2006).

53. L. Zhang, G. Cheng, C. Fu, React. Funct. Polym., 56, 167 (2003).

54. Y. Li, C. Dong, J. Chu, J. Qi, X. Li, Nanoscale, 3, 280 (2011). 


\section{Chapter 4}

\section{Conclusions and Future Work}

The main objective of the research was to integrate magnetic properties into MIP particles for the enhanced removal of EDCs from aqueous environment. With a novel synthetic method, the encapsulation of MIP layer on a magnetic core was achieved. The resultant core-shell hybrid had strong magnetic response. Consequently, a clean, rapid and cost-effective separation of estrogen-imprinted sorbent was possible simply by applying a magnetic field. However, the magnetic separation was performed in a nonflow mode at lab scale. Actual recycling operates in a large quantity in a continuous flow. To overcome the limitations of batch-wise separation process, high gradient magnetic separation and magnetically seeded filtration [1] appear to be useable in the future research for a continuous flow separation.

The encapsulation of polymeric layer on a magnetic core using aqueous ferrofluid involves the introduction of water into the polymerization system. One feature of this work is that the polymerization was realized in a hydro-organic medium with $50 \%$ aqueous content. The resultant M-MIP exhibited imprinting efficiency comparable to the MIPs synthesized in organic porogen(s). The development of water-compatible MIPs has been a notable area since MIPs prepared in organic solvent(s) exhibit reduced recognition ability in aqueous environment owing to the solvent memory effect and the weakened template-monomer interactions [2]. Attributed to the formation of a colloidal suspension, the hydro-organic medium provides a preferred environment for stabilizing magnetic particles and facilitating a rapid polymerization.

Ultrasonication assisted synthesis results in improved particle morphology and narrow size distribution. The finer M-MIP spherical particles were obtained in reduced particle diameter with ultrasonication process in comparison to the corresponding MIP 
prepared in conventional protocol [3]. A 2-h cycle represents a dramatic reduction in polymerization inputs, compared to 10-24 h conventional synthetic routes [4]. In the exploitation of encapsulation parameters, aqueous/organic ratio and the associated seed particles (MNPs) content have been found to improve coating efficiency. The importance of a compatible medium for a preferred polymer growth around the magnetic cores was demonstrated by high encapsulating efficiency. This synthetic technique was an interesting contribution to the various efforts in the development of new synthetic approaches for effective generation of MIPs [5]. However, the mechanism of this method needs further investigation for extended applications.

Binding performance of E2 M-MIP was verified for imprinting effect and adsorption capacity. In association with a $10 \%$ higher adsorption capacity of M-MIP than M-NIP, a $44 \%$ higher binding efficiency of M-MIP toward E2 than BPA indicates the presence of specific recognition sites in the MIP layer. The binding results validate the synthetic method and prove the adsorption specificity. The high selective affinity, however, also limits the application to steroid estrogens.

Binding properties of E2-BPA dual-template imprinted M-MIP were studied in the extraction of EDCs and interfering species. E2-BPA M-MIP exhibited a good adsorption capacity toward the templates and showed a high binding affinity toward other steroid and phenolic EDCs. The class-specific adsorption was verified by a significant difference in binding EDCs versus structurally different compounds (DFC, HQ and PH). The Freundlich isotherm model was found applicable to the M-MIP, indicating a heterogeneous distribution of the binding sites in the particle layer. The environmental variables (ionic strength, humic acid, $\mathrm{pH}$ and temperature) have shown only minor effects on M-MIP adsorption efficiency at environmentally relevant levels. A 7-cycle reuse showed good regenerating potential and stability. An ignorable reduction in binding capacity toward five EDCs after seven uses suggests a minor deterioration in 
recognition and magnetic properties. Looking forward, further study would be the exploration of appropriate imprinting techniques for binding various structurally related EDCs.

The research work described in this thesis on using magnetic MIP as selective sorbent with class-specificity presents the application potential for simultaneous removal of EDCs from aqueous environment. The effective synthesis of M-MIP using multipletemplate imprinting approach compasses future research on designing suitable M-MIPs in the context of alternative sorbents for environmental application in addressing the increasing concern on water quality associated with contamination from the emerging pollutants. 


\section{References}

1. T. Ying, S. Yiacoumi, C. Tsouris, Chem. Eng. Sci., 55, 1101 (2000).

2. L. Chen, S. Xu, J. Li, Chem. Soc. Rev., 40, 2922 (2011).

3. Y.Yang, E.P.C. Lai, Photochem. Photobiol. A, 213, 123 (2010).

4. M. Lasáková, P. Jandera, J. Separ. Sci., 32, 799 (2009).

5. A. Poma, A.P. Turner, S.A. Piletsky, Trends Biotechnol., 28, 629 (2010). 


\section{Appendices}

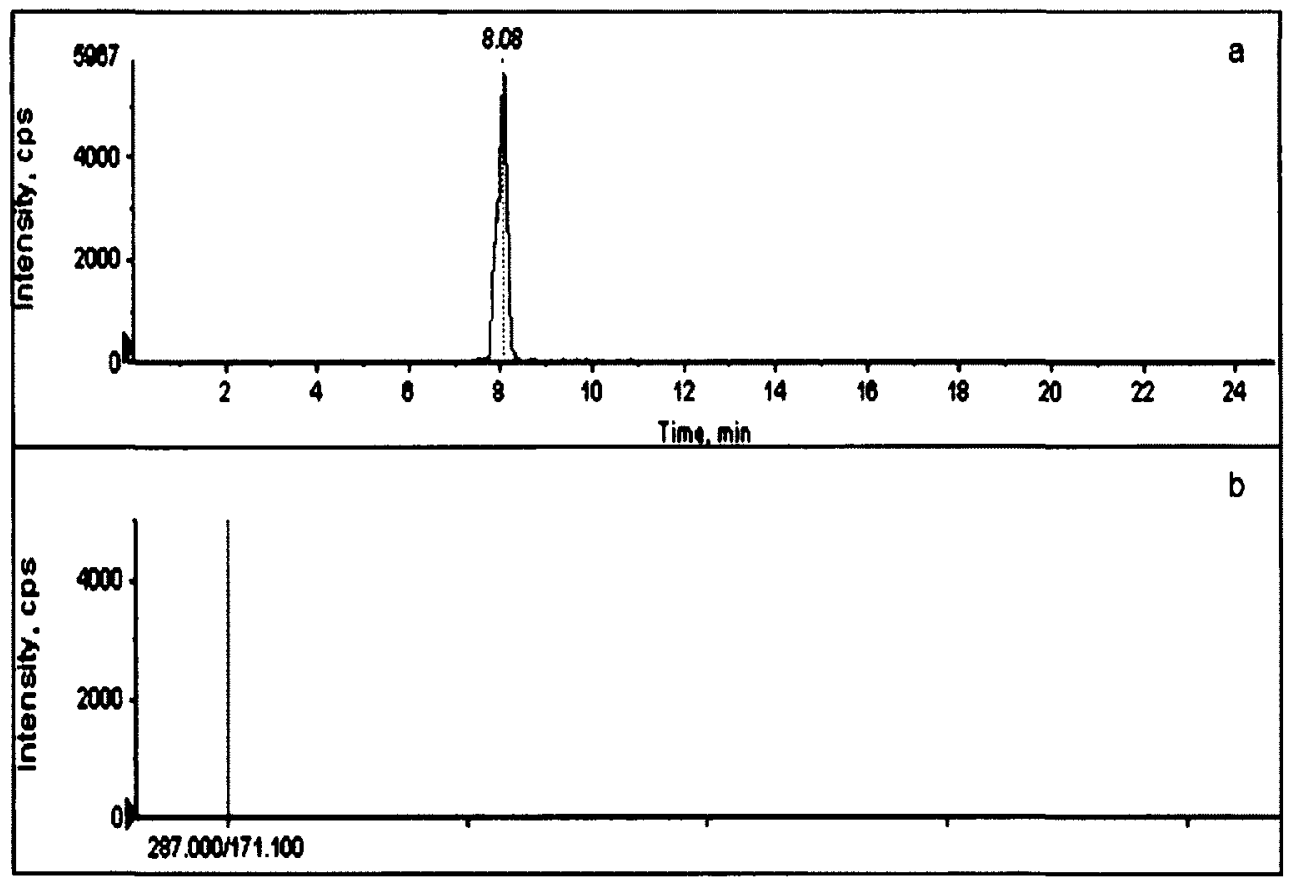

A. 1 LC-MS/MS chromatogram (a) and spectrum (b) of E3

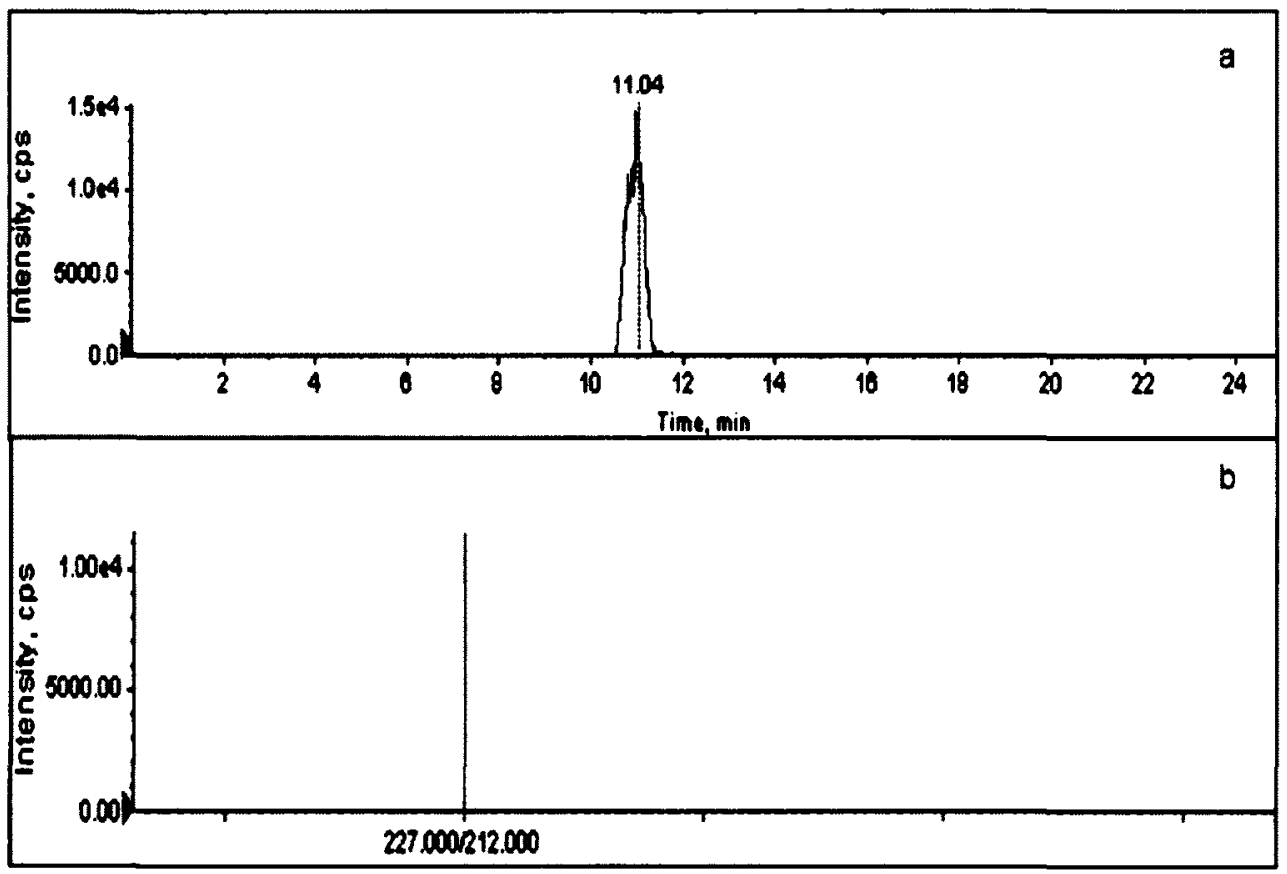

A.2 LC-MS/MS chromatogram (a) and spectrum (b) of BPA 


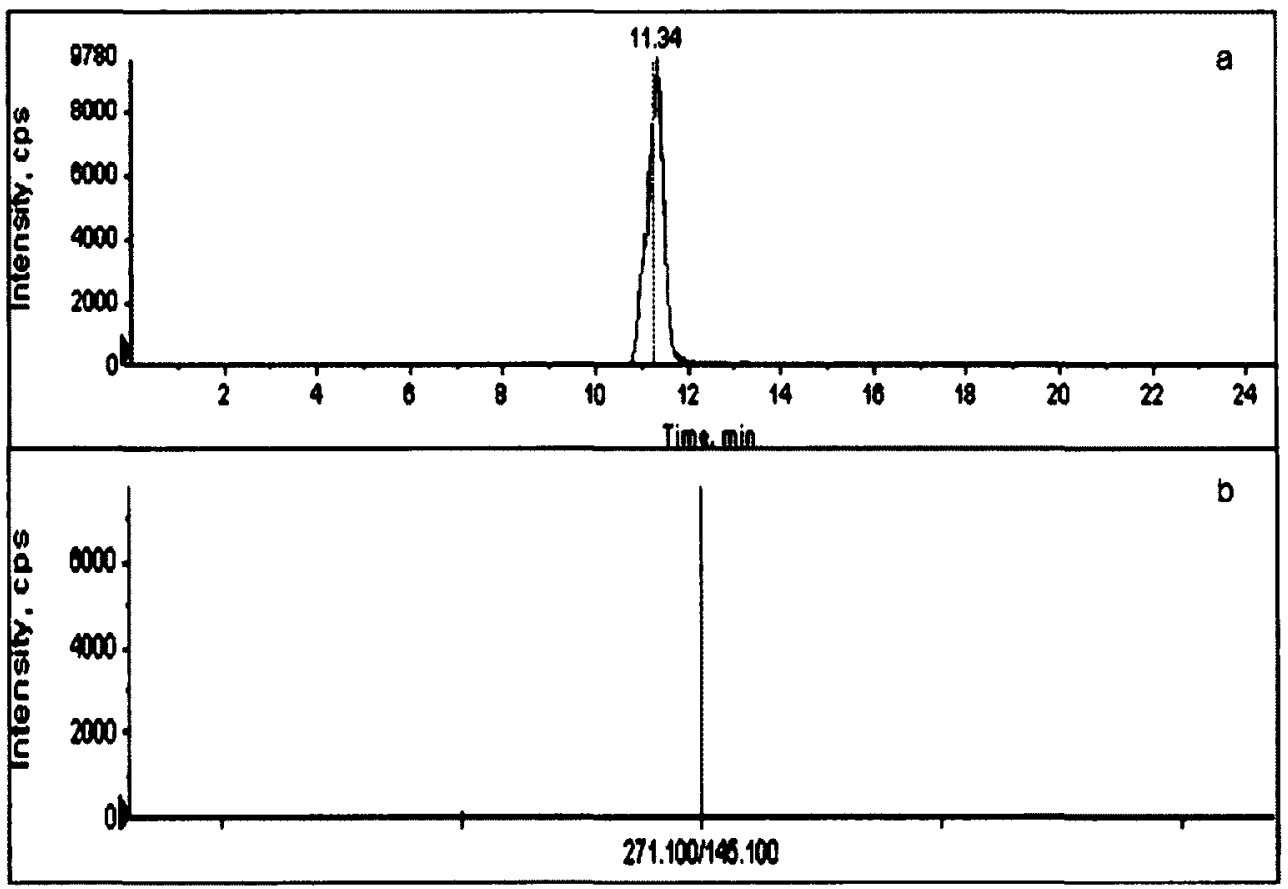

A.3 LC-MS/MS chromatogram (a) and spectrum (b) of E2

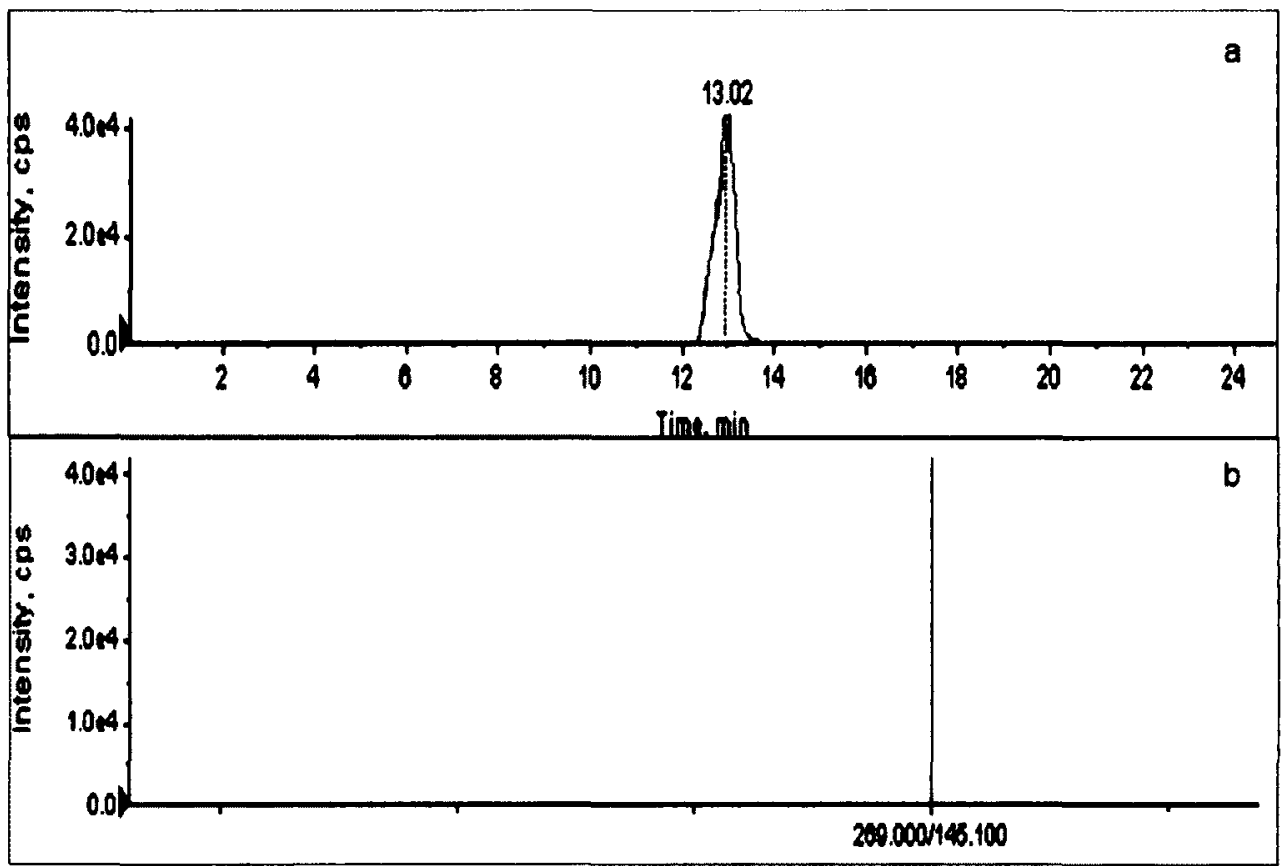

A.4 LC-MS/MS chromatogram (a) and spectrum (b) of E1 


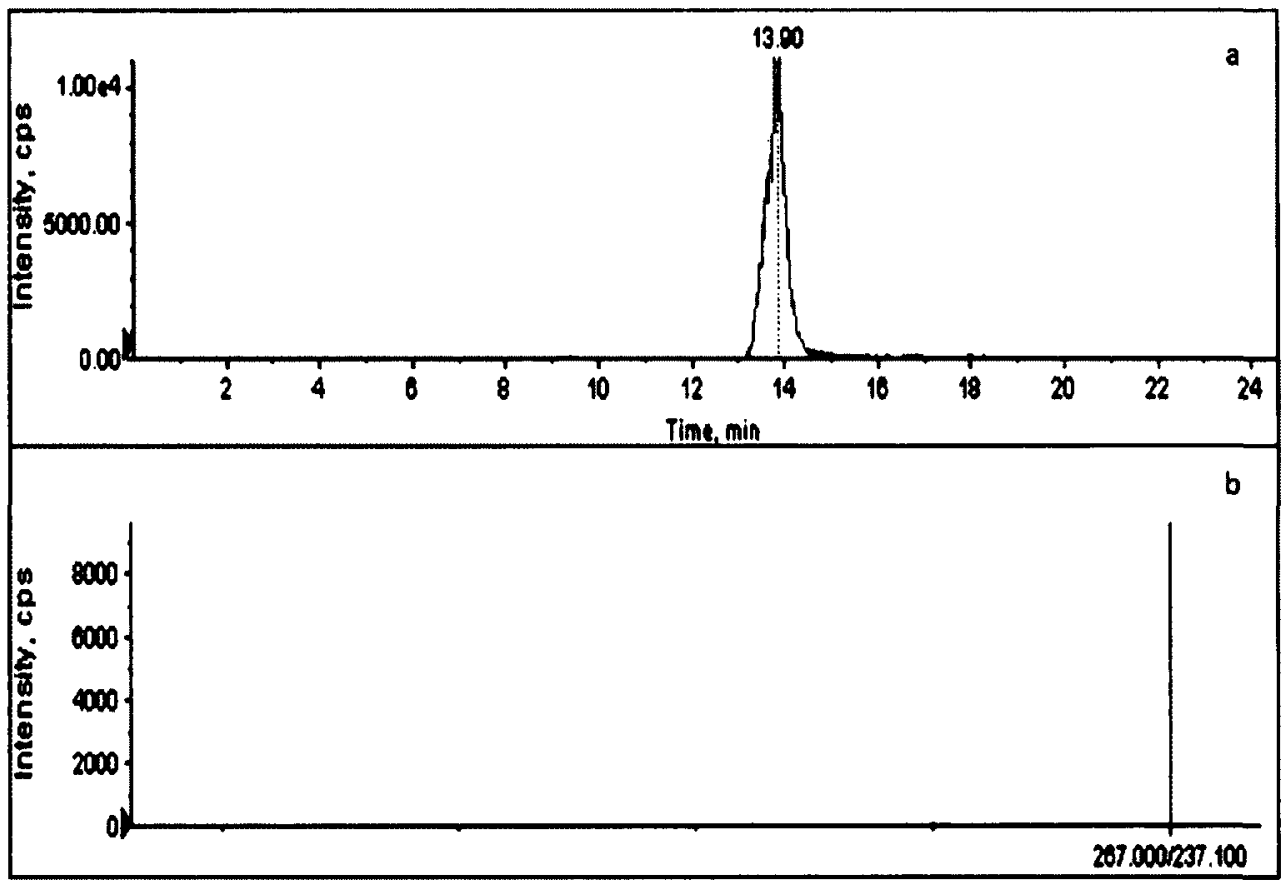

A.5 LC-MS/MS chromatogram (a) and spectrum (b) of DES

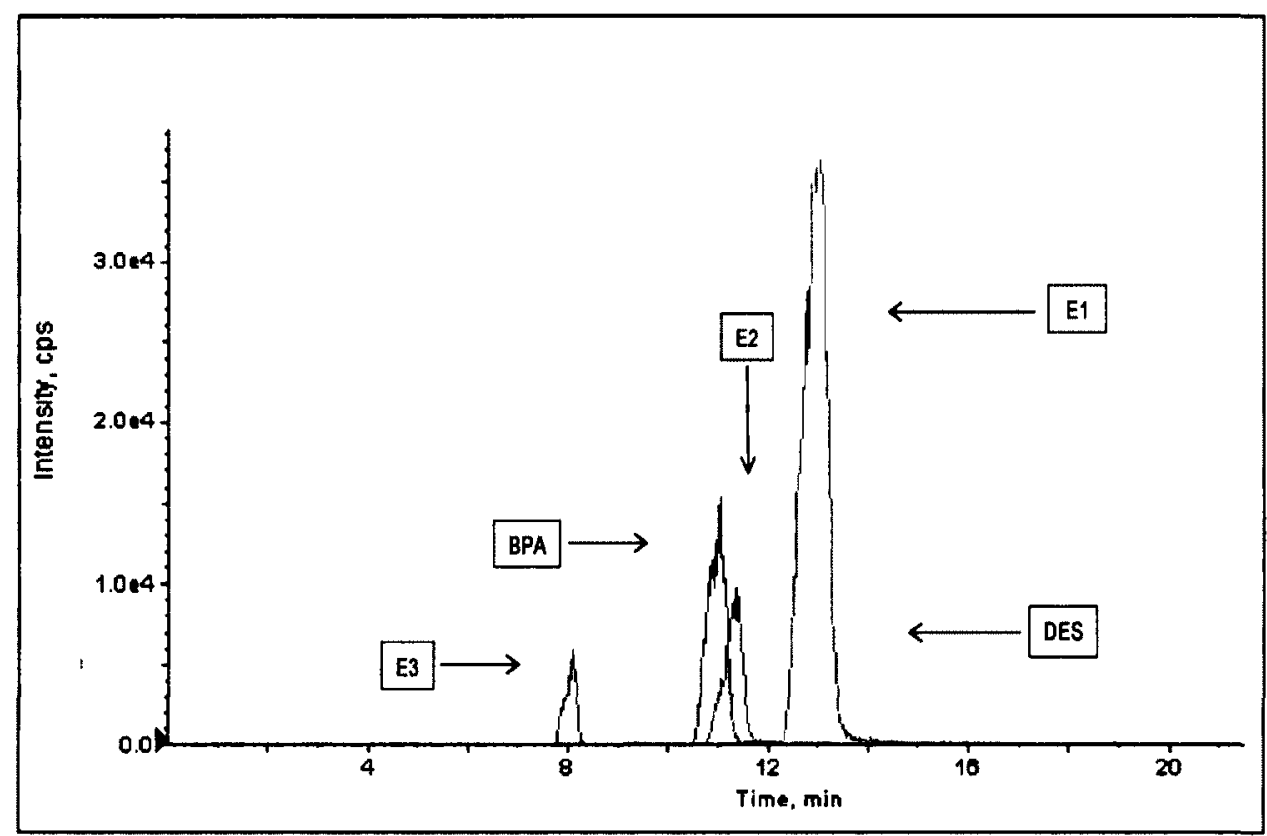

A. 6 LC-MS/MS chromatogram of the studied EDCs 